\title{
Drawing the line between kinematics and dynamics in special relativity
}

\author{
Michel Janssen \\ Program in the History of Science, Technology, and Medicine, \\ University of Minnesota
}

\begin{abstract}
In his book, Physical Relativity, Harvey Brown challenges the orthodox view that special relativity is preferable to those parts of Lorentz's classical ether theory it replaced because it revealed various phenomena that were given a dynamical explanation in Lorentz's theory to be purely kinematical. I want to defend this orthodoxy. The phenomena most commonly discussed in this context in the philosophical literature are length contraction and time dilation. I consider three other phenomena of this kind that played a role in the early reception of special relativity in the physics literature: the Fresnel drag effect in the Fizeau experiment, the velocity dependence of electron mass in $\beta$-ray deflection experiments by Kaufmann and others, and the delicately balanced torques on a moving charged capacitor in the Trouton-Noble experiment. I offer historical sketches of how Lorentz's dynamical explanations of these phenomena came to be replaced by their now standard kinematical explanations. I then take up the philosophical challenge posed by the work of Harvey Brown and Oliver Pooley and clarify how those kinematical explanations work.
\end{abstract}

Key words: Lorentz invariance, Minkowski space-time, kinematics, Trouton-Noble experiment, classical electron models, inference to the best explanation

\section{The cart and the horse}

"Einstein was the first physicist to formulate clearly the new kinematical foundation for all of physics inherent in Lorentz's electron theory" (Einstein, 19872006, Vol. 2, p. 253). This is how John Stachel and his associates characterized the transition from Lorentz's classical ether theory to Einstein's special theory

* Address: Tate Laboratory of Physics, 116 Church St. SE, Minneapolis, MN 55455, USA, Email: janss011@umn.edu

Preprint submitted to Elsevier Science 16 February 2008 
of relativity in the opening sentence of their editorial note on special relativity in Einstein's collected papers. Their statement nicely encapsulates the received view of this transition. Much of my own work, some of it in collaboration with Yuri Balashov, has been in defense of this orthodoxy (Janssen, 1995, 2002a; Balashov and Janssen, 2003). The agenda of Harvey Brown's work in this area, much of it in collaboration with Oliver Pooley, has been to question it (Brown and Pooley, 2001, 2006; Brown, 2005). Our central disagreement is summed up neatly - a little too neatly in fact - in the title of one of the sections of Physical Relativity, Brown's book-length defense of his heretical views on special relativity: "Minkowski space-time: the cart or the horse" (Brown, 2005, sec. 8.2; cf. Balashov and Janssen, 2003, p. 340-341). It is a dispute about the direction of the arrow of explanation connecting the symmetries of Minkowski space-time and the Lorentz invariance of the dynamical laws governing systems in Minkowski space-time. I argue that the space-time symmetries are the explanans and that the Lorentz invariance of the various laws is the explanandum; Brown argues that it is the other way around.

\subsection{Explanation and inference}

Before adding the necessary qualifications to this rough initial characterization of our disagreement, I want to address the rationale of continuing to frame the debate in terms of explanation. In his own response to (Brown, 2005), John Norton (2007) sidesteps "the explanatory issues that have dominated discussion elsewhere [since] they seem only to lead to futile disputes over just what it means to explain" (p. 5). Explanation is a notoriously tricky subject in philosophy of science, so why not follow Norton's lead and re-stage the debate in a different venue? Unfortunately, explanation is tied up with inference, which is absolutely central to the scientific enterprise.

In 1906, J. J. Thomson made an observation about the role of theories in physics that, I think, applies equally well to explanations. For a working physicist, Thomson wrote, a theory "is a policy rather than a creed." 2 Physicists use explanations not to adorn the results of their investigations with the elusive quality of understanding, but to help them come up with ideas for what to investigate next. They seek answers to why-questions in part no doubt for the sake of those answers themselves, but mostly to find clues and pointers in them for further research. Appropriating what Thomson said about theories, one can say that explanations are supposed to

connect or coordinate apparently diverse phenomena, and above all to suggest, stimulate, and direct experiment. It ought to furnish a compass which,

1 Brown's book was reviewed for this journal by Al Martínez (2007).

2 Quoted and discussed in (Smith, 2001, p. 63). 
if followed, will lead the observer further and further into previously unexplored regions. Whether these regions will be barren or fertile experience alone will decide; but, at any rate, one who is guided in this way will travel onward in a definite direction, and will not wander aimlessly to and fro (quoted from Smith, 2001, p. 63).

The last clause suggests that Thomson was content establishing the modest claim that relying on any explanation for guidance is better than invoking no explanations at all. It seems obvious, however, that the best explanations provide the most reliable guides. The challenge for philosophers of science is to work out criteria for what counts as a good explanation. What makes this challenge particularly pressing is precisely the heuristic role of explanations, not just in physics but in all of science.

One of my favorite examples comes from biology. In a book on the flora of Tasmania published in 1860, Hooker, one of Darwin's early confidants but no easy convert to his friend's nascent theory, stated that he had at long last switched from special creation to evolution through natural selection because he felt that the new theory supplied the more promising "means of penetrating the mystery which envelopes the history of species." ${ }^{3}$ Evolution would make for better botany, in Hooker's estimation, because it offered "a rational explanation" for various "attributes of organic life ... which are barren facts under the theory of special creations" (ibid.). Still, Hooker cautioned, the botanist predicating further research on these evolutionary explanations - to borrow a turn of phrase from George Smith (2001, p. 25) - should be "holding himself ready to lay it down when it shall prove as useless for the further advance of science, as the long serviceable theory of special creations ... now appears to me to be" (quoted from Bellon, 2006, p. 173).

The kind of reasoning that Hooker and, albeit more covertly, Thomson are referring to in these passages is known in the philosophical literature as 'inference to the best explanation' (IBE). Hooker is referring more specifically to a subspecies of IBE that I have dubbed 'common origin inference' (COI) (Janssen, 2002b). As Peter Lipton (2004) put it in his book on IBE: "the core idea of [IBE] is that explanatory considerations are a guide to inference" (p. 56). This then is why I am resisting Norton's suggestion to move the debate over the interpretation of special relativity out of the arena of explanation. As the case study in sec. 3 of this paper will illustrate most clearly, the seemingly arcane explanatory issue that Brown and I are arguing over can actually make a difference in scientific practice. Before I can make good on this claim, I need to characterize our positions more carefully.

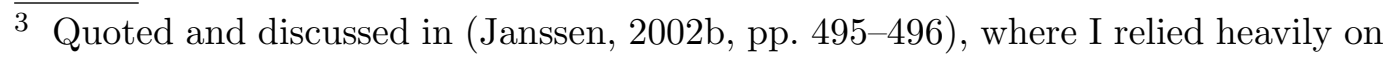
work since published as (Bellon, 2006). 


\subsection{Kinematics and dynamics}

Brown (2005, p. vii) makes it clear from the outset that he is not championing what is known as a neo-Lorentzian interpretation of special relativity. He is not proposing a return to the ether or to absolute time, two elements that Lorentz was never able to let go of. My work has focused on a comparison between the theories Lorentz and Einstein actually proposed. This comparison is nonetheless relevant to the evaluation of Brown's proposal. I have argued that the main objection against Lorentz's theory is not that it retains the ether or absolute time but that it seeks to provide dynamical explanations for a class of phenomena, namely all manifestations of Lorentz invariance, that special relativity revealed to be purely kinematical. That objection also applies to Brown's proposal. It is a mistake to keep looking for further explanation of a phenomenon once that phenomenon has convincingly been shown to be kinematical. What it means for a phenomenon to be kinematical, in the sense in which I want to use this term, is that it is nothing but a specific instance of some generic feature of the world, in the case of the phenomena examined in this paper instances of default spatio-temporal behavior. Unless one challenges the classification of the phenomenon as kinematical in this sense - and it is the universality of the relevant feature that will militate strongly against such reclassification - there is nothing more to learn from that particular phenomenon, neither about the specific system in which it occurs nor about the generic feature it instantiates. A call for further explanation is thus completely misplaced. The examples discussed in secs. 2-4 of this paper are meant to drive home this point. My disagreement with Brown is therefore ultimately about how to draw the line between kinematics and dynamics in special relativity.

In what has been the dominant tradition in philosophy of space and time "over the last three decades or so" (Brown, 2005, p. viii), any respectable philosophizing about space and time, be it about general relativity or about Aristotelian physics, starts with: "Let there be a differentiable manifold $M$ with geometric object fields $O_{i}$..." Several evangelists have been responsible for spreading this 'angle-brackets-M-O-sub-i' religion (referred to hereafter as $\left.\left\langle M, O_{i}\right\rangle\right)$. The Gospel According to St. Michael and the Gospel According to St. John have been particularly influential in bringing the philosophical community under its spell (Friedman, 1983; Earman, 1989). This tradition draws Brown's ire at several points in his book (2005, p. viii, p. 23) and I have limited patience for it myself. Still, it may be a useful exercise to formulate the kinematics-dynamics distinction in $\left\langle M, O_{i}\right\rangle$ terms (Janssen, 1995, sec. 2.3.5). If nothing else, it may help the $\left\langle M, O_{i}\right\rangle$ faithful see what all the fuss is about.

Consider a theory characterized by an $M$ (which fixes such basic things as the topology) and a set of $O_{i}$ 's. The $O_{i}$ 's can be divided into $A_{i}$ 's, which 'dress up' the bare manifold with its spatio-temporal properties (typically a metric and 
an affine connection), and $P_{i}^{\prime}$ 's, which encode the matter fields living on the manifold (Earman, 1989, p. 38, p. 45). The $P_{i}$ 's satisfy local field equations. In theories like general relativity, the $A_{i}$ 's do too, but in older theories they are fixed or absolute, which just means that they are the same in every model of the theory (i.e., in every configuration allowed by the field equations). This is the situation, for instance, with Minkowski space-time in special relativity, which in $\left\langle M, O_{i}\right\rangle$ terms is a bare manifold dressed up with a flat pseudo-Euclidean metric of Lorentzian signature. ${ }^{4}$ If the $A_{i}$ 's are absolute, it makes sense to call an effect kinematical if it instantiates properties described by the $A_{i}$ 's and dynamical if it instantiates properties described by the $P_{i}$ 's. That a free particle moves in a straight line is kinematical in this reckoning since such trajectories are the geodesics associated with the flat affine structure of Minkowski spacetime. Length contraction and time dilation are likewise kinematical effects in that they turn on comparisons of the lengths of certain line segments in the chrono-geometry of Minkowski space-time (Janssen, 2002a, p. 430). The emission of a photon is a dynamical effect because it instantiates a property of the electromagnetic field, which is one of the $P_{i}^{\text {'s. }}$.

Now, the description of any physical effect obviously involves both $A_{i}$ 's and $P_{i}$ 's, but that is perfectly compatible with this way of distinguishing between kinematical and dynamical effects. In particular, it is not a problem that one needs a physical system specified by the $P_{i}$ 's to measure properties described by the $A_{i}$ 's. It takes some material system that can serve as a rod, for instance, to measure length contraction. This truism does not change the fact that length contraction instantiates a property described by the $A_{i}$ 's. Neither does the stronger claim that without rods there would be no length contraction at all. The kinematics-dynamics distinction in terms of $A_{i}$ 's vs. $P_{i}$ 's can be made without turning Minkowski space-time, or any other space-time $\left\langle M, A_{i}\right\rangle$, into an autonomous substance that exists in addition to the physical systems characterized by the $P_{i}$ 's. One can make the same distinction if these physical systems, in the final analysis, are the bearers of the properties described by the $A_{i}$ 's.

This is the ontological constellation favored by Brown (2005): "I see the absolute geometrical structures of Minkowski space-time as parasitic on the relativistic properties of the dynamical matter fields" (p. 100). I sympathize with Brown here insofar as he is just saying that the laws governing the matter fields are the bearers of such properties as Lorentz invariance. That is simply an endorsement of a relational ontology of space-time. In this same spirit, I accept Brown and Pooley's (2006) slogan that Minkowski space-time is a "glorious non-entity." The pejorative 'parasitic' in the quotation above, however,

4 Minkowski's own work was done in the tradition of projective geometry associated with Klein's Erlangen program, whereas the differential geometry of $\left\langle M, O_{i}\right\rangle$ grew out of a very different tradition that goes back to Riemann (Norton, 1999). 
suggests that Brown wants to go further. In $\left\langle M, O_{i}\right\rangle$ jargon: Brown, it seems, wants to reduce both the $M$ and the $A_{i}$ 's to the $P_{i}$ 's, a proposal sharply criticized by Norton (2007). Here I side with Norton. The spatio-temporal notions encoded in Minkowski space-time do not have their origin in the particulars of some Lorentz-invariant matter theory or theories. Let me illustrate this point with an analogy. Most paintings are rectangular. The individual paintings are the bearers of this property. Yet, the answer to the question why they are rectangular is not to be found in any particular one of them. The answer to that question turns on factors beyond the individual paintings, having to do with artistic conventions or with the process of stretching cloth over a frame to make a canvas. Lorentz invariance is likewise a property that transcends individual laws even though they are the bearers of it.

Like Brown, I have no truck with the (dwindling?) denomination within the Church of $\left\langle M, O_{i}\right\rangle$ that goes by the name of manifold substantivalism. Members of this congregation are realists about the points of the bare manifold and see these points as the subjects for all field-theoretic predicates given by the $O_{i}$ 's. Given the general framework of $\left\langle M, O_{i}\right\rangle$, it is very seductive to read this ontology into the formalism. The resulting reification of $M$ is a typical example of what Stachel (1994, p. 149) has aptly called the "fetishism of mathematics." In fairness to $\left\langle M, O_{i}\right\rangle$, it must be said that work in this tradition has helped the relationist cause as well. $\left\langle M, O_{i}\right\rangle$ was instrumental in disseminating the hole argument, which has probably been the most persuasive and effective argument against manifold substantivalism (Earman, 1989, Ch. 9). Earman's book also took care of the centuries-old objection that absolute motion is incompatible with relationism by clearly separating the absolute/relative-motion distinction from the substantival/relational-ontology distinction.

It is important to separate the position I am defending in this paper from substantivalism. As Brown (2005) writes at one point: "The real issue is ... whether physical geometry ... when it is absolute and immune to perturbation as in Newtonian and Minkowskian space-time ... offers a causal explanation of anything" (p. 26, my emphasis). I claim that Minkowski space-time explains Lorentz invariance. For this to be a causal explanation, Minkowski space-time would have to be a substance with causal efficacy. Like Brown, I reject this view (Janssen, 2002b, p. 468). The sense in which Minkowski space-time explains Lorentz invariance is not causal but closer to the sense of explanation captured by the old covering-law or deductive-nomological account of explanation. It is partly to avoid misunderstandings of this sort that I want to define what I mean by kinematics without using $\left\langle M, O_{i}\right\rangle$ jargon.

Special relativity as a physical theory is agnostic about the ontology of spacetime. I want to argue that the orthodox version of this physical theory is preferable to the alternative proposed by Brown because it provides better guidance for further research. Given that my argument is ultimately about 
such methodological issues and not about ontology, it had better be independent of whether one is a relationist or a substantivalist about Minkowski space-time (Balashov and Janssen, 2003, p. 341, note 11). The challenge in this case is to produce an argument that works for the relationist. The substantivalist can always make that same argument work by reifying the relevant relations. I can thus join the debate with Brown on his own relationist turf without compromising the focus on methodological issues.

With a small but important modification, Brown's definition of (space-time) kinematics is perfectly adequate for my purposes. Commenting on the title of the first part of Einstein's 1905 paper on special relativity, "The kinematical part" (Einstein, 1905a), Brown (2005) defines it as "the universal behaviour of rods and clocks in motion" (p. 4). Given his operationalist bend at the time, Einstein clearly privileged physical systems serving as rods and clocks, but there is no reason for a restriction to such systems in the definition of kinematical. It is better to define (space-time) kinematics as the default spatiotemporal behavior of all physical systems (I prefer 'default' or 'generic' over 'universal'). Rods and clocks measure times and distances because they exhibit the default spatio-temporal behavior of all physical systems, not because they would be special probes sensing the fabric of space-time the way the 'waywiser' gracing the dust cover of Brown's book sensed the surface of the road as it was wheeled along the English countryside to measure distances in the days of old. ${ }^{5}$

Brown (2005) characterizes the Minkowski metric as "no more than a codification of the behaviour of rods and clocks" (p. 9). Once again, my only complaint is that this characterization privileges rods and clocks. I would say that Minkowski space-time (both its metric and its affine structure) encodes the default spatio-temporal behavior of all physical systems in a world in accordance with the laws of special relativity. Special relativity is completely agnostic about what inhabits or - to phrase it more awkwardly but in a way that may be more congenial to a relationist-carries Minkowski space-time. All the theory has to say about systems inhabiting/carrying Minkowski spacetime is that their spatio-temporal behavior must be in accordance with the rules it encodes. This requirement is automatically met if the system obeys Lorentz-invariant laws. It is in this sense that the Lorentz invariance of all dynamical laws is explained by space-time being Minkowskian rather than the other way around. This explanation can be put to work in that it considerably narrows the field of acceptable dynamical laws by requiring them

$\overline{5}$ Brown ridicules this 'tracing grooves in space-time'-imagery. His target, I presume, is substantivalism. Most commentators seem to agree that he is attacking a straw man. John Earman (private communication) calls this straw man "the really freaky spacetime freak." Like any good caricature, however, Brown's caricature of the substantivalist does have its value. 
to be Lorentz invariant. Special relativity imposes a kinematical constraint on all dynamical laws. This kinematical constraint is an example of what Marc Lange (2007) calls a meta-law. A meta-law cannot be derived from mere ordinary laws (which is not to say that we cannot look for a deeper explanation of the meta-law). In Lange's language, this is the error that Lorentz made a century ago and that Brown now invites us to repeat.

\subsection{History and philosophy}

After going over three concrete examples of phenomena that are purely kinematical in (orthodox) special relativity (secs. 2-4), I return to my dispute with Brown in the concluding section of this paper (sec. 5). With these concrete examples in hand, I shall be in a better position to articulate and defend the usefulness and naturalness of the way in which I want to draw the line between kinematical and dynamical effects. The notion of 'kinematical' that I want to promote is relevant in other contexts as well. I am thinking in particular of Heisenberg's (1925) use of the term in the title of the famous Umdeutung paper with which he laid the foundation for matrix mechanics, "On the quantum-mechanical reinterpretation of kinematical and mechanical relations" (my emphasis), and of recent work on the foundations of quantum mechanics by Jeffrey Bub and Itamar Pitowsky (2007). To bring out this wider relevance, I need to distinguish between a broad and a narrow sense of kinematical. A phenomenon is kinematical in the broad sense if it is independent of the details of the dynamics. It is kinematical in the narrow sense if it is an example of standard spatio-temporal behavior. As the terminology suggests, if a phenomenon is kinematical in the narrow sense, it is a fortiori kinematical in the broad sense. I shall use the phenomena discussed in secs. 2-4 to illustrate this distinction. Although philosophers of space and time only use the term 'kinematical' in the narrow sense, physicists routinely use it in the broad sense as well.

At several points in this introduction I already expressed strong sympathy with Brown's views. The three historical sections that make up the bulk of this paper will testify to what is probably the deepest affinity between his work and mine. We both practice a brand of philosophy of physics that is strongly informed by (conceptual) history of physics. This provides an additional reason for our shared dislike of $\left\langle M, O_{i}\right\rangle$. There is nothing to be gained from an $\left\langle M, O_{i}\right\rangle$ treatment of the texts documenting the transition from the electrodynamics of the last decade of the 19th century to the relativistic physics of the first decade

of the 20th century. That does not mean that one has to follow these texts slavishly. I recognize the value of standardizing notation and choice of units, of explaining results in terms familiar to modern readers, or, more trivially, of translating German quotations into English. 
It is probably no coincidence that I draw on the work of a different group of physicists for my philosophical arguments than Brown draws on for his. Lorentz, Einstein, and Minkowski, unsurprisingly, play a central role both in Brown's book and in my paper. Brown's main focus, however, is on such late-Victorian Maxwellians as FitzGerald, Heaviside, and Larmor. Although Larmor is a key figure in one of my case studies (see sec. 4), I focus on the continent, especially Germany, and on a slightly later period (1901-1911). In particular, I look at the shift from Abraham's electromagnetic view of nature to Laue's relativistic continuum mechanics. To a large extent this was a shift from dynamics to kinematics, in both the broad and the narrow sense (Janssen and Mecklenburg, 2007, sec. 7).

I not only look at different physicists, I also look at different physics. I avoid such staples of the literature on the history and philosophy of special relativity as the Michelson-Morley and Kennedy-Thorndike experiments and the length contraction and clock retardation hypotheses. The dynamical explanation of the null result of Michelson and Morley by FitzGerald, Lorentz, and Larmor provides grist for the mill of Brown's philosophical argument. I base mine on the analysis of three other experiments that have not received much attention thus far in the discussion of special relativity in the philosophy of space and time literature, even though all three of them played a role in the early reception and elaboration of the theory. A subsidiary goal of my paper is to bring these experiments and their analysis by Lorentz, Abraham, Laue and others to the attention of philosophers working in this area. ${ }^{6}$ I have written the sections covering this material with this goal in mind, highlighting the philosophically salient elements and providing only a minimum of historical context. For more careful historical treatments of the relevant episodes and additional references to the historical literature, I refer to some of my other work.

In sec. 2, I examine the so-called Fresnel drag coefficient famously put to the test in the Fizeau experiment of 1851 (Janssen and Stachel, 2004). Fresnel introduced the drag coefficient in 1818 to account for refraction at surfaces moving through the ether. In 1892, Lorentz explained this "drag" effect in terms of the interaction of light with charged particles inside transparent media. Three years later, he showed (in effect) that the drag coefficient would automatically be part of any Lorentz-invariant theory of refraction. In 1907, Laue showed that this is because it results from a straightforward application of the relativistic addition theorem of velocities. This episode thus makes for

$\overline{6}$ More examples could probably be added. It would be interesting, for instance, although this is beyond the scope of this paper, to look at experiments by Röntgen, Rowland, Eichenwald, H. A. Wilson, and M. Wilson (Pauli, 1921, sec. 36( $\alpha)$ ) from the same 'dynamics vs. kinematics'-angle from which I look at experiments by Fizeau, Kaufmann, Trouton, and Noble in secs. 2-4. 
an ideal warm-up example of an effect that went from being classified as dynamical to being classified as kinematical (first in the broad and then in the narrow sense).

In sec. 3 and 4, I turn to two considerably more complicated examples, both in terms of the physics involved and in terms of their history. From a relativistic point of view, both examples revolve around the transformation properties of the four-momentum of spatially extended systems (Rohrlich, 1960, 1965). Sec. 3 deals with the velocity dependence of electron mass measured in a series of experiments by Kaufmann and others in the first two decades of the 20th century (Janssen and Mecklenburg, 2007). This episode illustrates how physicists can be led down the garden path-to borrow another turn of phrase from Smith (2001, p. 23) - by the demand for a dynamical explanation of phenomena that are purely kinematical. I shall argue that the velocity dependence of mass, like the Fresnel drag coefficient, is kinematical both in the broad and in the narrow sense. What complicates matters in this case is that the kinematics-dynamics distinction tends to get entangled with Einstein's (1919) famous distinction between principle theories and constructive theories (Brown, 2005, sec. 5.2). Part of my rebuttal of Brown will be to disentangle the two.

Sec. 4 deals with the torque that Trouton and Noble tried to detect in 1903 on a charged capacitor moving through the ether (Janssen, 1995). The standard relativistic explanation of the negative result of this experiment is that there are two delicately balanced torques, one coming from the electromagnetic forces between the charges on the plates and one coming from the intermolecular forces stabilizing the system. It turns out that one can simply define these torques out of existence in special relativity by adopting an alternative convention for choosing spatial hyperplanes in the definition of the four-momentum of such spatially extended systems as the capacitor and its electromagnetic field. The same is true for the flow of energy and momentum between the capacitor and its electromagnetic field in a variant on the Trouton-Noble experiment in which a moving capacitor is set spinning. I shall contrast this kinematical effect with the flow of energy and momentum from the battery to the capacitor in the Trouton experiment, a largely forgotten prequel to the Trouton-Noble experiment (Janssen, 2003). This way of defining torques and energy and momentum exchanges out of existence suggests a general criterion for classifying an effect as kinematical (in the narrow sense), which dovetails nicely with the way in which I want to draw the line between kinematics and dynamics in special relativity (Janssen, 1995, sec. 2.3.5). 


\section{The Fizeau experiment and the Fresnel drag coefficient.}

\subsection{Aberration, refraction, and the Fresnel drag coefficient}

Physicists in the 19th century took it to be completely self-evident that light waves, like all other waves, need a medium for their propagation. Since light can reach us from the farthest recesses of the universe, this medium, the luminiferous ether, had to be omnipresent. In 1804, Young pointed out that the phenomenon of stellar aberration, discovered by Bradley in the 1720s, indicated that this universal ether be immobile, i.e., that the earth and other ponderable matter move through it without disturbing it in the least. Fig. 1 illustrates stellar aberration for a star directly overhead. The situation is drawn from the point of view of the ether. The solid vertical line through

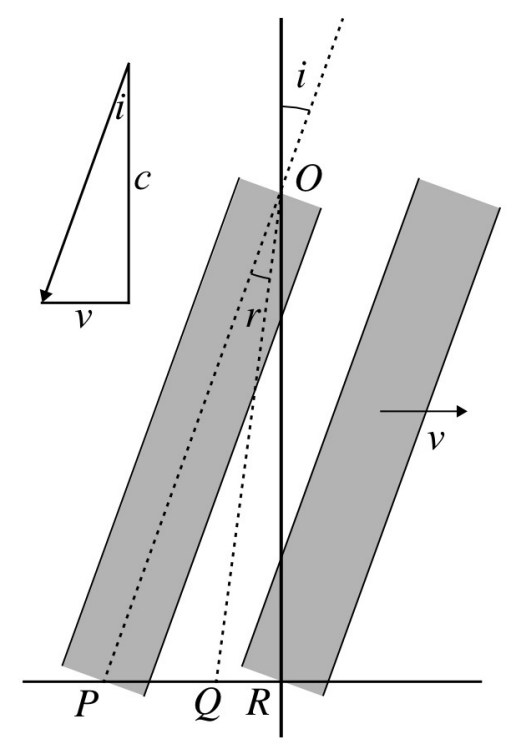

Fig. 1. Stellar aberration

$O$ and $R$ (ignore the dashed lines for the moment) represents a light ray (more accurately: the normal to a plane wave front) traveling from the star to the earth at velocity $c$. The shaded rectangles represent two snapshots of a telescope moving with the earth at velocity $v$, the first as the light enters at $O$, the second as it exits at $R$. For the telescope to collect the light of this star, it must be tilted at an angle, called the aberration angle and labeled $i$ in the figure. This means that an observer on earth will see the star in the direction indicated by the dashed line through $O$ and $P$. Drawing a vector diagram for the two components of the velocity of the light with respect to a terrestrial observer, one sees that the aberration angle is given by $\tan i=v / c$ (since $v / c \approx 10^{-4}$, the angle $i$ is actually much smaller than the drawing in Fig. 1 suggests). Any currents in the ether would add more components to 
the velocity of light and change the aberration angle. Since $v / c$ was clearly the observed value (for a star directly overhead), Young concluded that the universal ether had to be immobile.

In 1818, however, Fresnel argued that in some situations ether does get dragged along by matter. In the early part of the 19th century, wave theorists assumed that the index of refraction $n$ was proportional to the square root of the ether density $\rho$. Moving transparent substances, Fresnel assumed, would not affect the universal ether in the space they traveled through but would carry excess ether along with them to preserve the ether density inside. The weighted average of the velocities of these two types of ether, the unaffected and the dragged-along, is a fraction $\rho_{\text {excess }} / \rho_{\text {total }}$ of the velocity with which the substance is moving through the ether. Since $\rho_{\text {excess }}=\rho_{\text {total }}-\rho_{\text {vacuum }}$ and $\rho_{\text {total }} / \rho_{\text {vacuum }}=n^{2}$, this fraction is equal to $1-1 / n^{2}$. This expression became known as the Fresnel drag coefficient. In 1846, Stokes suggested an alternative mechanism in which transparent media moving through the ether drags along all ether inside of it with this fraction.

No matter how one envisions this ether drag, Fresnel showed that the component it adds to the velocity of light is necessary to explain why the presumed motion of the earth with respect to the universal ether does not affect the outcome of refraction experiments. This extra velocity component ensures that, to first order in $v / c$ (and greater experimental accuracy was not attainable until much later in the century), refraction at the surface of a body moving through the ether will follow Snell's law, $\sin i=n \sin r$ (where $i$ is the angle of incidence and $r$ is the angle of refraction), from the point of view of someone moving with the refracting body. A lens in a telescope is an example of a refracting body in motion through the ether. In the simple derivation of the formula for the aberration angle above, it was tacitly assumed that the observer moving with the telescope can appeal to Snell's laws to describe the refraction in the lenses of the telescope. That assumption, Fresnel showed, is not as innocuous as it may sound. It would not be true without the extra velocity component resulting from the Fresnel drag effect. With this extra component, however, no first-order refraction experiment can ever reveal the earth's motion through the ether. In 1871, for instance, Airy found that filling the tube of his telescope with water did not affect the aberration angle.

A more primitive version of Airy's experiment can be used to derive Fresnel's result for the special case in which the angle of incidence is $0^{\circ}$ for the observer moving with the refracting body. Imagine that the shaded rectangles in Fig. 1 represent two snapshots of a piece of glass with flat surfaces at the top and the bottom. From the point of view of the ether, the light ray striking the surface at $O$ makes an angle $i$ with the normal, the dashed line through $O$ and $P$. If Snell's law were to hold from the ether's perspective, as would be the case if no ether drag were assumed, the refracted ray would follow the dashed 
line segment $O Q$ at an angle $r<i$ with the normal. For an observer moving with the glass, because of aberration (no matter whether the light source is terrestrial or celestial), the light ray strikes the surface at $O$ perpendicularly. If Snell's law holds from this observer's perspective, the light thus goes straight through $\left(r^{\prime}=i^{\prime}=0^{\circ}\right)$, which from the perspective from which Fig. 1 is drawn means that the refracted ray follows the solid line segment $O R$. As the light is traveling through the glass, it must therefore be dragged from $O Q$ to $O R$. Suppose it takes the light an amount of time $\Delta t$ to get from $O$ to $R$. In that case,

$$
O Q=(c / n) \Delta t, \quad P R=v \Delta t, \quad Q R=f v \Delta t
$$

where $f$ at this point is some unknown fraction of $v$. Since the angles $i$ and $r$ are very small, their tangents and sines can be used interchangeably and the angle $P Q O$ is almost a right angle so that $\tan r$ can be set equal to $P Q / O Q$. Substituting this value into Snell's law in the form $\tan i=n \tan r$ and using that the aberration angle $i$ satisfies $\tan i=v / c$, one finds:

$$
\frac{v}{c} \approx n \frac{P Q}{O Q}=n \frac{P R-Q R}{O Q} .
$$

Substituting the expressions in Eq. (1) in Eq. (2), one finds:

$$
\frac{v}{c} \approx \frac{v}{c} n^{2}(1-f)
$$

It follows that, to order $v / c, f$ must be equal to $1-1 / n^{2}$, which is just the Fresnel drag coefficient.

Direct confirmation of the "drag" effect, or so it seemed, was provided in 1851 when Fizeau did an interference experiment that convincingly showed that flowing water drags along light waves with about half its velocity, which is roughly the value of the Fresnel drag coefficient for water. In 1886, as a prelude to their famous ether drift experiment the following year, Michelson and Morley repeated Fizeau's experiment and found the same result. In the period 1914-1927, Zeeman measured the velocity of light in various moving liquids and solids and confirmed the Fresnel drag coefficient (with a small correction term due to Lorentz) with much greater accuracy than either Fizeau or Michelson and Morley (Zeeman, 1927).

\subsection{Lorentz's two derivations of the Fresnel drag coefficient in the $1890 \mathrm{~s}$}

Although the formula for the Fresnel drag effect was widely accepted in the 19th century, the proposals for the physical mechanism behind it-be it Fres-

nel's picture of full drag of some ether or Stokes's picture of partial drag of all ether - were not. Stachel (2005, pp. 6-8) quotes statements by Fizeau in 1851 and by Ketteler, Mascart, and Veltmann in the early 1870s to this effect. The 
main objection to the literal interpretation of the Fresnel drag coefficient in terms of ether drag was connected to a more general problem facing theoretical accounts of the phenomenon of optical dispersion, the differential refraction of light of different colors familiar from rainbows and prisms. ${ }^{7}$ Dispersion theory ought to furnish a formula showing how the index of refraction depends on the frequency of the refracted light. In the early part of the 19th century, as I already mentioned, the index of refraction was assumed to be proportional to the square root of the ether density. This means that substances must carry different amounts of ether for different colors of light. This implies, if the Fresnel drag coefficient is interpreted literally, that substances would have to drag along ether with different fractions of their velocity for different colors.

What eventually led to the abandonment of these simple theories of refraction and dispersion in terms of variable ether density was that they could not account for the phenomenon of anomalous dispersion, in which the index of refraction for part(s) of the spectrum decreases rather than increases with frequency. The phenomenon had been noticed by early pioneers in photography but did not receive serious attention from physicists until the 1870s. At that point, Sellmeier, Helmholtz and others began to develop a new type of dispersion theory in which the behavior of light in transparent media is explained in terms of the interaction of the light waves with small harmonicallybound particles with resonance frequencies at the absorption frequencies of the material. It is in the vicinity of these frequencies that dispersion becomes anomalous. Originally, these theories were purely mechanical, but in the early 1890s they were reworked in terms of electromagnetic waves interacting with electrically charged particles, later to be identified as electrons. The most sophisticated theory along these lines was the one proposed by Lorentz (1892a) in a monograph-length paper on Maxwell's electromagnetic theory and its application to moving bodies. The ether is completely immobile in this theory and has the same density everywhere. The index of refraction is related not to ether density but to the concentration of charged oscillators.

Lorentz's 190-page treatise is divided into seven chapters and an appendix. The behavior of light in dielectric media is the topic of the final two chapters. Ch. VI, on media at rest in the ether, takes up 24 pages (Lorentz, 1892a, pp. 474-497). Ch. VII, on media in motion through the ether, takes up 30 pages (ibid., pp. 498-527). In Ch. VI, Lorentz derives the equations governing the propagation of light in a medium at rest in the ether and shows that they have solutions describing waves traveling with velocity $c / n$ through the ether, where $n$ can be expressed in terms of properties of Lorentz's microscopic model of the medium. In Ch. VII, he derives the analogous equations for a medium moving through the ether with some velocity $v$, using a co-moving frame of reference

\footnotetext{
7 For a brief discussion of 19th-century dispersion theory and references to further literature on this topic, see (Duncan and Janssen, 2007a, sec. 3.1).
} 
(related to a frame at rest in the ether through a Galilean transformation). Lorentz (1892a, pp. 524-527) shows that these equations allow waves with velocity $(c / n)-\left(v / n^{2}\right)$ in the direction of motion of the medium. The velocity of these waves with respect to the ether is $(c / n)+\left(1-1 / n^{2}\right) v$, in accordance with Fresnel's formula.

Physicists had been struggling with dispersion since the days of Newton, so it was a tremendous success for Lorentz's theory that it gave a reasonably satisfactory account not just of normal but also of anomalous dispersion. ${ }^{8}$ What especially inspired confidence in Lorentz's theory was that it gave the Fresnel drag coefficient without introducing any actual ether drag. This was a triumph for the theory on a par with the explanation of the normal Zeeman effect half a decade later. Einstein still rehearsed the final steps of Lorentz's 1892 derivation of the Fresnel drag coefficient in an unpublished review article on special relativity twenty years later as well as in courses on special relativity in 1914-15 and 1918-19 in Berlin, as can be gleaned from his lecture notes (Einstein, 1987-2006, Vol. 7, p. 279, note 7). What makes this all the more remarkable is that Einstein did not cover-neither in these three documents nor in any other document that I am aware of - a far simpler derivation of the Fresnel drag coefficient that Lorentz gave in 1895 and that is much closer in spirit to special relativity. Norton (2004, pp. 87-92) conjectures that this 1895 derivation was one of the stepping stones on Einstein's path to special relativity and is thus forced to explain away that Einstein covered the 1892 derivation in these documents without so much as a hint at the 1895 one.

The 1895 derivation is given in two short sections of a book on the electrodynamics of moving bodies known in the historical literature as the Versuch (Lorentz, 1895, secs. 68-69, pp. 95-97). It is an application of the so-called theorem of corresponding states that Lorentz first introduced in this book. Later versions of this theorem (Lorentz, 1899, 1904b) will play an important role in secs. 3-4. In modern terms, these theorems express, though initially only partially and approximately, the Lorentz invariance of Maxwell's equations. At the beginning of sec. 3.1, I shall formulate the theorem in a form specifically tailored to its use in this paper. In this section, I want to stay closer to Lorentz's own reasoning.

Lorentz first subjected Maxwell's equations to what would now be called a Galilean transformation from a frame of reference with Cartesian coordinates $\left(x_{0}, y_{0}, z_{0}\right)$ at rest in the ether to a frame with coordinates $(x, y, z)$ moving through the ether with velocity $\mathbf{v}$. It will be convenient to assume that this velocity is in the $x$-direction. Lorentz now replaced the electric and magnetic fields and the time coordinate with auxiliary quantities such that, as long as

\footnotetext{
8 Only two decades later, the old quantum theory would pull the rug out from under Lorentz's account of dispersion (Duncan and Janssen, 2007a, sec. 3).
} 
quantities $v^{2} / c^{2}$ and smaller are neglected, the equations in the moving frame have the same form as Maxwell's equations in a frame at rest in the ether. To first order in $v / c$, the quantities replacing the fields and the time $t$ in the moving frame are just what would now be called the Lorentz transforms of the fields and the time $t_{0}=t$ in the frame at rest. The auxiliary time variable thus depends on position and Lorentz gave it the suggestive name "local time" (Lorentz, 1895, p. 81). For the frame moving through the ether at velocity $v$ in the $x$-direction, it is given by:

$$
t^{\prime} \equiv t-\left(v / c^{2}\right) x
$$

Lorentz used the embryonic Lorentz invariance of Maxwell's equations to show that one could never detect the earth's motion through the ether with a firstorder experiment in optics that ultimately boils down to the observation of a pattern of brightness and darkness. Any such experiment performed on earth, in near-uniform motion through the ether, would give the same result that one would find if one could somehow perform the experiment at rest in the ether.

Given how broad this class of experiments is, the argument showing that the theorem of corresponding states predicts negative results for all of them is surprisingly simple. ${ }^{9}$ The auxiliary fields at a point with coordinates $\mathbf{x}$ and at local time $t^{\prime}$ in the experiment on earth will have the same values as the real fields in the experiment at rest in the ether for the same values of the coordinates $\mathbf{x}_{0}$ and the real time $t_{0}$. To describe a pattern of brightness and darkness it suffices to specify where the fields are large averaged over times that are long compared to the periods of the light waves used and where these averages vanish. The components of the auxiliary fields are linear combinations of components of the real fields (cf. Eq. (15) below). They vanish or are large wherever and whenever the real fields are. Since patterns of brightness and darkness can only be defined on time scales that are large compared to the periods of the light waves producing them, local time and real time can be used interchangeably. Combining these observations, one arrives at the conclusion that if there is a bright (dark) spot at point $\mathbf{x}_{0}$ in the experiment at rest in the ether, then there will likewise be bright (dark) spot at the corresponding point $\mathbf{x}$ in the experiment on earth. The experiment will not reveal the earth's motion through the ether.

The class of optical experiments covered by Lorentz's argument clearly includes refraction experiments. Fresnel had shown that refraction experiments will reveal motion through the ether according to the immobile-ether theory unless the Fresnel drag coefficient is added to it. Lorentz's theory must thus imply the Fresnel drag coefficient. In fact, it is a consequence of the embryonic Lorentz invariance of Maxwell's equations that Lorentz established with his

$\overline{9}$ But I suppress one key assumption that Lorentz tacitly made (see note 12 below). 
theorem of corresponding states. As Lorentz (1895, secs. 56-58) showed explicitly, it follows directly from the expression for local time. In Ch. VI of his 1892 treatise, Lorentz had shown that Maxwell's equations can serve as the basis for a theory explaining why light travels at velocity $c / n$ through a transparent medium with refractive index $n$ at rest in the ether. The components of the electric and magnetic fields of a light wave traveling in the $x$-direction all depend in the same way on $\mathbf{x}_{0}$ and $t_{0}$ via the combination

$$
t_{0}-\frac{x_{0}}{c / n}
$$

Now consider the same transparent medium moving through the ether with velocity $v$ in the $x$-direction. Replacing the real fields, the coordinates $\mathbf{x}_{0}$, and the real time $t_{0}$ in the description of a light wave in the medium at rest in the ether by the auxiliary fields, the coordinates $\mathbf{x}$, and the local time $t^{\prime}$ of the moving frame, one arrives at a description of a light wave in the same medium in motion through the ether. For a wave in the $x$-direction, the components of the auxiliary fields all depend on $t^{\prime}$ and $x$ via

$$
t^{\prime}-\frac{x}{c / n}
$$

The same is true for the components of the real fields, which are just linear combinations of the components of the auxiliary fields. Using expression (4) for $t^{\prime}$, one finds that they all depend on $t$ and $x$ via

$$
t-\left(\frac{v}{c^{2}}+\frac{n}{c}\right) x
$$

Taking the reciprocal of the expression in parentheses, one finds that the light wave in the moving medium has velocity

$$
\left(\frac{v}{c^{2}}+\frac{n}{c}\right)^{-1}=\frac{c / n}{1+(v / c n)} \approx \frac{c}{n}-\frac{v}{n^{2}}
$$

in the $x$-direction with respect to the medium. The medium itself is moving through the ether with velocity $v$ in the $x$-direction. So, to order $v / c$, the light wave in the moving medium has velocity

$$
\frac{c}{n}+\left(1-\frac{1}{n^{2}}\right) v
$$

with respect to the ether, in accordance with Fresnel's formula.

Lorentz's simple derivation of the Fresnel drag coefficient of 1895 (see Eqs. (5)-(9)) rendered the lengthy calculations in Ch. VII of his 1892 treatise superfluous. The new derivation made it clear that it suffices to derive from Maxwell's equations that light has velocity $c / n$ in a medium at rest in the ether with refractive index $n$ (Lorentz, 1892a, Ch. VI) and to show that Maxwell's 
equations are invariant under Lorentz transformations, at least to first order in $v / c$ and for the kind of charge distributions involved (Lorentz, 1895, secs. 56-58). Although Lorentz himself saw it merely as a convenient shortcut for his derivation of 1892, he had thus achieved a good deal more with his new derivation of 1895. In the terms that I introduced in sec. 1.3, the 1895 derivation shows that the Fresnel drag coefficient is kinematical in the broad sense of being independent of the details of the dynamics.

\subsection{Laue's derivation of the Fresnel drag coefficient from the relativistic ad- dition theorem for velocities}

It was left to Laue in 1907 to show that the Fresnel drag coefficient is also kinematical in the narrow sense of having to do with standard spatio-temporal behavior in special relativity. Laue (1907) showed that the drag coefficient is a direct consequence of the relativistic addition theorem of velocities. Einstein (1905a) derived the theorem in his first paper on special relativity, but missed this important application of it. This is another omission that is hard to square with Norton's (2004) conjecture about the importance of Lorentz's derivation of the Fresnel drag coefficient from the expression for local time for Einstein's path to special relativity. As both Einstein and Poincaré recognized, the $x$ dependent term in Lorentz's expression for local time reflects the relativity of simultaneity. This is the only effect that matters in Laue's derivation of the Fresnel drag coefficient. To derive the addition theorem of velocities in full generality, one also needs to take into account the effects of time dilation and length contraction, but those are effects of second order in $v / c$ while the validity of the Fresnel drag coefficient is restricted to first order.

Consider light moving through a medium moving at velocity $v$, both in the (positive) $x$-direction. For an observer moving with this medium, the light has velocity $u^{\prime}=c / n$. Let $\Delta x^{\prime}$ be the distance covered by the light in the time $\Delta t^{\prime}$ from the point of view of the co-moving observer. In other words,

$$
u^{\prime} \equiv \frac{\Delta x^{\prime}}{\Delta t^{\prime}}=\frac{c}{n}
$$

What is the velocity $u$ of the light with respect to the medium for an observer with respect to whom the medium is moving at velocity $v$ in the $x$-direction? To order $v / c$, the two observers agree on the distance covered: $\Delta x=\Delta x^{\prime}$. However, they do not agree on the time it takes to cover this distance. This is because they disagree about the synchronization of the clocks at the end points of the interval $\Delta x=\Delta x^{\prime} . \Delta t^{\prime}$ in Eq. (10) is determined on the assumption that these two clocks are properly synchronized according to the co-moving observer. For the other observer, as follows from Eq. (4), the clock on the left is fast compared to the clock on the right by an amount of $\left(v / c^{2}\right) \Delta x$. This 
amount needs to added to the time $\Delta t^{\prime}$ reported by the co-moving observer. According to the observer with respect to whom the medium is moving, the velocity $u$ of the light with respect to the medium is thus given by:

$$
u \equiv \frac{\Delta x}{\Delta t} \approx \frac{\Delta x^{\prime}}{\Delta t^{\prime}+\left(v / c^{2}\right) \Delta x^{\prime}}=\frac{u^{\prime}}{1+\left(v / c^{2}\right) u^{\prime}} .
$$

If $c / n$ is substituted for $u^{\prime}$, this reduces to (cf. Eq. (8))

$$
u \approx \frac{c / n}{1+(v / n c)} \approx \frac{c}{n}-\frac{v}{n^{2}} .
$$

It follows that, to order $v / c$, the light has velocity (cf. Eq. (9))

$$
u+v=\frac{c}{n}+\left(1-\frac{1}{n^{2}}\right) v
$$

with respect to the observer for which the medium is moving at velocity $v$ in the $x$-direction. This concludes the proof that the Fresnel drag coefficient is a direct consequence of the relativity of simultaneity.

Laue's 1907 derivation of the Fresnel drag coefficient is mathematically equivalent to Lorentz's 1895 derivation (compare Eqs. (10)-(13) to Eqs. (5)-(9)). Laue's derivation, however, clearly brings out the meaning of the $x$-dependent term in Lorentz's local time in terms of the relativity of simultaneity. It also shows, in the unkind glare of hindsight, that it was a mistake to look for a dynamical explanation of the extra velocity component that Fresnel showed was needed for a satisfactory account of refraction in moving media (cf. Eqs. (1)-(3)). Ketteler, Veltmann, and Mascart were right in the 1870s to use Fresnel's formula but to ignore its dynamical explanation in terms of ether drag. And physicists in the 1890s were wrong to count Lorentz's dynamical explanation of 1892 as further evidence for his impressive electrodynamical theory of refraction and dispersion. The Fresnel drag coefficient did not call for a new dynamics but for a new space-time kinematics.

\subsection{Drawing the line between kinematics and dynamics in accounting for the Fresnel drag coefficient}

The Fresnel drag coefficient was introduced in 1818 to ensure that refraction in a medium moving through the ether obeys Snell's law of refraction from the point of view of a co-moving observer. Fresnel and, nearly thirty years later, Stokes tried to derive the drag coefficient from dynamical models in which a moving medium drags along at least some of the ether inside of it. The problem with these models was that the moving medium would have to drag along different amounts of ether for different colors because of the phenomenon of 
dispersion. The models for moving media inherited this problem from models for media at rest in ether in which the index of refraction was interpreted in terms of ether density. To account for dispersion, a medium at rest would have to contain different amounts of ether. This problem for media at rest was solved by the development of a new generation of dynamical models in which the index of refraction was interpreted in terms of oscillators inside the medium. Mechanical versions of this model emerged in the 1870s and Lorentz proposed an electromagnetic version of it in 1892. He also produced a model for moving media. This model involved no actual ether drag and avoided the absurdities of the models of Fresnel and Stokes. Lorentz, however, could have saved himself the trouble of producing a separate model for moving media. In modern terms, his model for a medium in motion is simply the Lorentz-boosted version of his model for a medium at rest. The features of the model for a medium in motion, including the Fresnel 'drag' effect, follow from the Lorentz invariance of the model for a medium at rest. This model was Lorentz invariant because it was based on Maxwell's equations. In fact, any Lorentz-invariant model for a medium at rest that reproduces Snell's law of refraction entails the Fresnel 'drag' effect in a medium in motion. The effect is thus kinematical in the broad sense of being independent of the details of the dynamics. Lorentz, in effect, showed this in 1895 when he derived the Fresnel drag coefficient from ( $i$ ) the assumption that it is possible to derive from a Lorentz-invariant theory like Maxwell's that light has velocity $c / n$ in a medium at rest (where $n$ is the index of refraction) and (ii) the expression $t-\left(v / c^{2}\right) x$ for local time in his theorem of corresponding states.

The kinematical nature of the Fresnel drag coefficient (both in the broad and in the the narrow sense) was emphasized by Einstein in his popular book on relativity. After presenting Laue's derivation of the drag coefficient from the relativistic addition theorem of velocities, he wrote:

a theory of this phenomenon was given by H. A. Lorentz [1892a] long before the statement of the theory of relativity. This theory was of a purely electrodynamical nature, and was obtained by the use of particular hypotheses as to the electromagnetic structure of matter (Einstein, 1917, p. 41).

In another passage in the book (to which I shall return in sec. 3.5), Einstein explicitly stated that special relativity gives the Fresnel drag coefficient "without the necessity of drawing on hypotheses as to the physical nature of the liquid" (ibid., p. 51). As Norton suggests, Einstein may have had an ulterior motive in mentioning Lorentz's derivation of 1892 on several occasions but not his derivation of 1895 (see sec. 2.2): "Einstein may have wanted to contrast Lorentz's dynamical derivation of 1892 with the kinematical derivation in special relativity, conveniently passing over Lorentz's 1895 result" (Norton, 2004, p. 91). Norton disparages Lorentz's 1892 derivation as "quite unilluminating, demonstrating only that a rather cumbersome and opaque application 
of Maxwell's equations to the propagation of electromagnetic waves in moving media yields the Fresnel drag," while praising the 1895 one as "a much simpler, essentially kinematical derivation" (ibid.). ${ }^{10}$

Laue's derivation of the Fresnel drag coefficient shows that the drag effect is also kinematical in the narrow sense of reflecting default spatio-temporal behavior. Contrary to what its origin in the analysis of refraction and aberration suggests, the Fresnel drag coefficient carries no information whatsoever about the physics of light in transparent media other than that it is in accordance with the general rules for the spatio-temporal behavior of systems inhabiting/carrying Minkowski space-time. More specifically, the Fresnel drag coefficient reflects that the velocities involved when light propagates through a moving medium add the way all velocities add in special relativity.

\section{The Kaufmann experiments and the velocity dependence of elec- tron mass}

\subsection{The velocity dependence of electron mass and the absence of ether drift}

By the late 1890s it appeared increasingly unlikely that any experiment, no matter how accurate, would ever detect ether drift. Lorentz (1899, sec. 9) thus explored how the treatment of negative results of first-order optical etherdrift experiments in the Versuch (Lorentz, 1895) could be extended to higher powers of $v / c$. The centerpiece of this extension was an exact version of the Versuch's theorem of corresponding states. In modern terms, this theorem is just the statement that Maxwell's equations are invariant under Lorentz transformations. In 1899, Lorentz only considered the source-free equations and the equations for a few special charge distributions. Moreover, the transformations he considered still contained an overall scale factor $\varepsilon$, which is an

\footnotetext{
${ }^{10}$ I essentially agree with Norton's assessment, but I do want to register a few reservations. First, I want to emphasize that Lorentz's (1892a) by Norton's lights equally "cumbersome and opaque" application of Maxwell's equations to the propagation of electromagnetic waves in media at rest in the ether in Ch. VI of his treatise was a milestone in the checkered history of dispersion theory. Moreover, even Ch. VII on moving media was of considerable value. In this chapter Lorentz showed for the first time in nearly three quarters of a century that a coherent account of the physics behind the Fresnel drag coefficient was possible. That he did not recognize right away that his was only one possible account hardly diminishes this achievement. Finally, I find it hard to believe that Einstein would pass over Lorentz's 1895 derivation in silence if that derivation really was as important as Norton conjectures it was for Einstein's path to special relativity.
} 
undetermined function of $v$. Following the systematic exposition of the theory in (Lorentz, 1904b), I shall use $l \equiv 1 / \varepsilon$ instead.

For the purposes of this paper, the theorem of corresponding states can be stated as follows: ${ }^{11}$ if an electromagnetic field configuration, described by the electric field $\mathbf{E}^{\prime}$ and the magnetic field $\mathbf{B}^{\prime}$ as functions of the space-time coordinates $\left(\mathbf{x}^{\prime}, t^{\prime}\right)$, is a solution of Maxwell's equations, then what up to the scale factor $l$ is just the Lorentz-boosted version of this configuration, described by $\mathbf{E}$ and $\mathbf{B}$ as functions of $(\mathbf{x}, t)$, is also a solution. In accordance with this formulation of the theorem in terms of active transformations, both primed and unprimed coordinates refer to a frame of reference at rest in the ether. Lorentz referred the boosted configurations to a co-moving frame related to the frame at rest in the ether via a Galilean transformation (cf. sec. 2.2). For a Lorentz boost with velocity $v$ in the $x$-direction, $(\mathbf{x}, t)$ and $\left(\mathbf{x}^{\prime}, t^{\prime}\right)$ are related via

$$
c t=(\gamma / l)\left(c t^{\prime}+\beta x^{\prime}\right), \quad x=(\gamma / l)\left(x^{\prime}+\beta c t^{\prime}\right), \quad y=y^{\prime} / l, \quad z=z^{\prime} / l,
$$

with $\beta \equiv v / c$ and $\gamma \equiv 1 / \sqrt{1-\beta^{2}}$. Lorentz (1899, p. 439) stipulated somewhat arbitrarily that $l$ can only differ from unity by a quantity of order $v^{2} / c^{2}$. This ensures that corresponding field configurations at rest and in motion with respect to the ether have the same spatial dimensions to first order in $v / c$. The fields at corresponding points in the two situations are related via:

$$
\mathbf{E}=l^{2} \operatorname{diag}(1, \gamma, \gamma)\left(\mathbf{E}^{\prime}-\mathbf{v} \times \mathbf{B}^{\prime}\right), \quad \mathbf{B}=l^{2} \operatorname{diag}(1, \gamma, \gamma)\left(\mathbf{B}^{\prime}+\left(1 / c^{2}\right) \mathbf{v} \times \mathbf{E}^{\prime}\right),
$$

where $\operatorname{diag}(a, b, c)$ is shorthand for a diagonal matrix with diagonal elements $a, b$, and $c$. One obtains the inverse transformations by interchanging primed and unprimed quantities and by replacing $v$ by $-v$ and $l$ by $l^{-1}$. For $l=1$ the transformation is completely symmetric. This observation provides a simple argument for setting $l=1$. Both Einstein (1905a) and Poincaré (1906) availed themselves of variants of this argument. Lorentz (1904b) decided on $l=1$ on the basis of a roundabout dynamical argument (see sec. 3.3).

Applied to the source-free Maxwell equations, the theorem of corresponding states by itself does not predict that optical experiments cannot detect uniform motion with respect to the ether. This is because the equations only cover the patterns of brightness and darkness associated with the electromagnetic field configurations, and not the optical components with which such patterns are created and observed (lamps, mirrors, lenses, gratings, slits, diaphragms, screens etc.). It follows from Eq. (14), that the dimensions of a pattern of brightness and darkness in a frame at rest in the ether must be divided by $\gamma l$ in the $x$-direction and by $l$ in the $y$ - and $z$-directions to obtain the dimensions

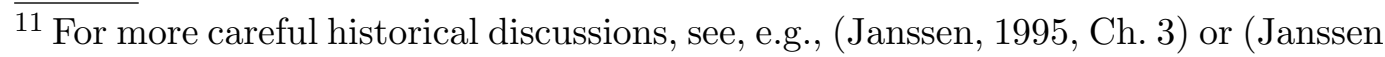
and Stachel, 2004). 
of the corresponding pattern of brightness and darkness in a frame in motion through ether with velocity $\mathbf{v}=(v, 0,0)$. Similarly, the periods of the light waves in the situation at rest must be multiplied by $\gamma / l$ to obtain the periods in the corresponding situation in motion. For $l=1$, these are the familiar length-contraction and time-dilation effects of special relativity.

A co-moving observer should be able to detect these differences unless the dimensions and periods of the material systems interacting with the electromagnetic field in these experiments depend on $v$ in the same way as the dimensions and periods of the patterns of brightness and darkness do. To predict in full generality and to all orders of $(v / c)$ that a moving observer cannot detect ether drift with an optical experiment that ultimately boils down to the observation of a pattern of brightness and darkness, Lorentz thus had to add a far-reaching physical assumption to his mathematical theorem about Maxwell's equations. He had to assume that a material system producing a particular field configuration at rest in the ether would automatically turn into the system producing the corresponding state of that field configuration in a frame moving through the ether if it were carefully and slowly accelerated to the velocity of that frame. ${ }^{12}$ In conjunction with the exact theorem of corresponding states, this new assumption does predict that a broad class of optical experiments will fail to detect ether drift. As Lorentz (1899, p. 440; 1904b, p. 22) recognized, the new assumption was a generalization of the contraction hypothesis he and FitzGerald had introduced several years earlier to explain the negative result of one such experiment, that of Michelson and Morley. Elsewhere I have therefore dubbed it the 'generalized contraction hypothesis' (Janssen, 1995, 2002b). As Brown (2005, pp. 49-50) emphasizes, it would be better to talk about "deformation" hypotheses. It is only for $l=1$ that the only deformation will be a contraction in the direction of motion. In relativistic terms, the generalized deformation hypothesis amounts to the assumption that the laws effectively governing material systems are Lorentz invariant (modulo $l$ ), just as the laws (i.e., Maxwell's equations) governing the electromagnetic fields with which they interact. Hence, by adopting the generalized deformation hypothesis, Lorentz implicitly decreed a number of exemptions to the Galilean-invariant laws of Newtonian mechanics that were supposed to have jurisdiction over matter in his theory.

Contrary to the strictures of Newtonian mechanics, lengths, periods, forces, and masses in Lorentz's theory all acquire the velocity dependence now famil-

\footnotetext{
${ }^{12}$ Lorentz tacitly made this same assumption in applications of his first-order theorem of corresponding states in 1895. The assumption was much more innocuous in that context because it did not require any deviations from the laws of Newtonian mechanics governing the material systems. Most deviations are of order $v^{2} / c^{2}$ and in the kind of situations Lorentz considered deviations of order $v / c$ (such as the term distinguishing local time from real time) played only a minor role.
} 
iar from special relativity. For lengths and periods this can be seen directly from Eq. (14). In the case of forces and masses it calls for additional arguments. Lorentz (1899, pp. 440-442) considered the electrons - or "ions" as he still called them-generating light waves. He compared their oscillations in a system at rest in the ether to those in a corresponding state in a system moving through the ether. As part of a plausibility argument for the Lorentz-FitzGerald contraction, Lorentz (1892b, 1895) had already made the assumption that all forces are affected by motion through the ether the same way Coulomb forces are (Janssen, 1995, sec. 3.2). In relativistic terms, he thus assumed that all forces transform as Coulomb forces under Lorentz transformations (modulo $l$ ). The force $\mathbf{F}$ on an electron moving through the ether with velocity $v$ in the $x$-direction is related to the force $\mathbf{F}^{\prime}$ on that same electron in the corresponding state at rest in the ether via (Lorentz, 1899, p. 441; 1904b, p. 18, Eq. (21)):

$$
\mathbf{F}=l^{2} \operatorname{diag}(1,1 / \gamma, 1 / \gamma) \mathbf{F}^{\prime} .
$$

Lorentz also found a simple relation between the corresponding accelerations. From a relativistic point of view, he only took into account the effects of length contraction and time dilation (modulo l). Lorentz (1899, p. 441; 1904b, p. 27, Eq. (33)) thus arrived at:

$$
\mathbf{a}=\frac{d^{2} \mathbf{x}}{d t^{2}}=\frac{(1 / l) \operatorname{diag}(1 / \gamma, 1,1)}{(\gamma / l)^{2}} \frac{d^{2} \mathbf{x}^{\prime}}{d t^{\prime 2}}=l \operatorname{diag}\left(\frac{1}{\gamma^{3}}, \frac{1}{\gamma^{2}}, \frac{1}{\gamma^{2}}\right) \mathbf{a}^{\prime} .
$$

In general, the relativity of simultaneity complicates matters, but Lorentz's simple relation (17) carries over intact to special relativity as long as the velocity with which the electron is oscillating is negligible compared to the velocity $v$ with which the system as a whole is moving through the ether. Planck (1906a) was the first to derive the general transformation law for acceleration in special relativity (Zahar, 1989, sec. 7.1). In the system at rest in the ether, the electron satisfies $\mathbf{F}^{\prime}=m^{\prime} \mathbf{a}^{\prime}$, where $m^{\prime}$ is the mass of the electron at rest in the ether (i.e., in modern terms, $m^{\prime}$ is the electron's rest mass). Using the inverse of the transformation laws (16) and (17) to write $\mathbf{F}^{\prime}=m^{\prime} \mathbf{a}^{\prime}$ in terms of $\mathbf{F}$ and $\mathbf{a}$, one finds:

$$
\frac{1}{l^{2}} \operatorname{diag}(1, \gamma, \gamma) \mathbf{F}=\frac{1}{l} \operatorname{diag}\left(\gamma^{3}, \gamma^{2}, \gamma^{2}\right) m^{\prime} \mathbf{a},
$$

which reduces to

$$
\mathbf{F}=l \operatorname{diag}\left(\gamma^{3}, \gamma, \gamma\right) m^{\prime} \mathbf{a} .
$$

It follows that the electron's oscillation in the moving frame only satisfies Newton's second law if its mass depends on its velocity and if its 'longitudinal mass' $\left(m_{/}\right)$, the inertia for acceleration in the direction of motion, differs from its 'tranverse mass' $\left(m_{\perp}\right)$, the inertia for acceleration perpendicular to the direction of motion. More specifically, $m_{/ /}$and $m_{\perp}$ should satisfy (Lorentz, 
1899, p. 442; 1904b, p. 27):

$$
m_{/ /}=l \gamma^{3} m^{\prime}, \quad m_{\perp}=l \gamma m^{\prime} .
$$

Both $m_{/ /}$and $m_{\perp}$ increase dramatically as the velocity of the electron approaches the velocity of light.

The generalized deformation hypothesis thus forced a serious departure from Newtonian mechanics. In Lorentz's theory, the one Newtonian mass, the constant ratio of force and acceleration, bifurcates into two velocity-dependent quantities. Lorentz (1899) admitted that this move "seems very startling at first sight" (p. 442). "Nevertheless," he added, "we need not wholly reject it. Indeed, as is well known, the effective mass of an ion depends on what goes on in the aether; it may therefore very well be altered by a translation and even to different degrees for vibrations of different directions" (ibid.). Lorentz did not provide any references to the literature at this point, but (J. J.) Thomson had suggested as early as 1881 that the inertia of a charged sphere increases as a result of the interaction with its self-field, a result elaborated and improved on by Heaviside in 1889 and Searle in 1897 (Miller, 1981, p. 43).

This idea, born in Victorian England, was about to be pursued vigorously by several prominent physicists on the continent. Inspired by the successes of Lorentz's electromagnetic theory and by experiments confirming that the mass of the electron does indeed increase with velocity, they set out to develop an electromagnetic account of this velocity dependence. Although they brought their own unanticipated rewards, these efforts turned out to be misguided. As Einstein (1905a, sec. 10) first suggested, Eqs. (20) for $l=1$ simply give the generic velocity dependence of mass in a new relativistic mechanics. ${ }^{13}$ They provide no insight whatsoever into the origin of the electron's mass.

\subsection{The velocity dependence of electron mass, the electromagnetic view of nature, and the Kaufmann experiments}

In a Festschrift for Lorentz, Wien (1900) proclaimed what would come to be known as the electromagnetic view of nature (McCormmach, 1970). To put Wien's proposal in terms of a pair of admittedly anachronistic slogans: everything is made of electromagnetic fields and Maxwell's equations are the theory of everything. Abraham, a Privatdozent in Göttingen at the time and a former student of Planck, emerged as the leader of this electromagnetic program

\footnotetext{
${ }^{13}$ Instead of using Eq. (16), Einstein set $\mathbf{F}=\mathbf{F}^{\prime}$ and found that $m_{\perp}=\gamma^{2} m^{\prime}$. Planck (1906a) adopted Eq. (16) and found $m_{\perp}=\gamma m^{\prime}$. When his 1905 paper was reprinted in 1911, Einstein added a footnote conceding that this definition of $\mathbf{F}$ is preferable to his own (Einstein, 1905a, p. 63).
} 
(Goldberg, 1970). The reduction of inertia to the interaction of charges with their self-fields became its central problem. (For the strict adherent to the program the charges themselves were nothing but singularities in the field.) The touchstone for success in this reduction program was whether the predictions for the velocity dependence of the electron's mass agreed with experiment.

Abraham showed that the electromagnetic mass of a given charge distribution can be derived either from the energy or from the momentum of its selffield (Janssen and Mecklenburg, 2007, sec. 2.2). Electromagnetic energy is the integral over all of space of the electromagnetic energy density $u_{\mathrm{EM}}(\mathbf{x}, t):{ }^{14}$

$$
U_{\mathrm{EM}}(t)=\int u_{\mathrm{EM}}(\mathbf{x}, t) d^{3} x=\int\left(\frac{1}{2} \varepsilon_{0} \mathbf{E}^{2}+\frac{1}{2} \mu_{0}^{-1} \mathbf{B}^{2}\right) d^{3} x,
$$

where $\varepsilon_{0}$ and $\mu_{0}$ are constants, which satisfy $c=1 / \sqrt{\varepsilon_{0} \mu_{0}}$. I use the letter $U$ for energy, reserving the letter $E$ for the electric field. Likewise, electromagnetic momentum, a concept introduced by Abraham (1902a, 1903), is the integral over all of space of the electromagnetic momentum density $\mathbf{p}_{\mathrm{EM}}(\mathbf{x}, t)$ :

$$
\mathbf{P}_{\mathrm{EM}}(t)=\int \mathbf{p}_{\mathrm{EM}}(\mathbf{x}, t) d^{3} x=\int \varepsilon_{0}(\mathbf{E} \times \mathbf{B}) d^{3} x .
$$

Like Lorentz in 1899, Abraham distinguished between longitudinal and transverse mass. In fact, this terminology is his (Abraham, 1903, pp. 150-152). Both can be computed from the electromagnetic momentum. The transverse mass is given by:

$$
m_{\perp}=\frac{P_{\mathrm{EM}}}{v}
$$

the longitudinal mass by:

$$
m_{/ /}=\frac{d P_{\mathrm{EM}}}{d v} .
$$

The latter can also be derived from the electromagnetic energy:

$$
m_{/ /}=\frac{1}{v} \frac{d U_{\mathrm{EM}}}{d v}
$$

Note that if the Newtonian expressions for momentum and kinetic energy, $p=m_{\mathrm{N}} v$ and $U_{\text {kin }}=\frac{1}{2} m_{\mathrm{N}} v^{2}$, are substituted for $P_{\mathrm{EM}}$ and $U_{\mathrm{EM}}$, respectively, Eqs. (23) $-(25)$ simply reproduce the Newtonian result $m_{\perp}=m_{/ /}=m_{\mathrm{N}}$.

Abraham (1902a,b) also proposed a concrete model of the electron. In this model, the electron is a rigid spherical surface charge distribution. It always retains its shape regardless of its state of motion with respect to the ether. So Abraham, despite his avowed ambition to reduce Newtonian mechanics to electrodynamics, tacitly accepted the Newtonian concepts of space and time. Calculating the longitudinal and transverse mass for this model with the help

\footnotetext{
${ }^{14}$ I use SI units throughout this paper.
} 
of Eqs. (23)-(25), he found expressions far more complicated than Lorentz's (see Eq. (20)) but qualitatively similar in that the two components of the electron's mass increase sharply with velocity.

In 1901, Kaufmann, one of Abraham's colleagues in Göttingen, had started a series of experiments in which he used combinations of electric and magnetic fields to deflect electrons produced by $\beta$-decay. ${ }^{15} \mathrm{He}$ found that the (transverse) mass of electrons in $\beta$-radiation, which reach velocities of over $90 \%$ the speed of light, was much greater than the mass of electrons in cathode rays, which never reached velocities greater than about $30 \%$ the speed of light. He interpreted this velocity dependence as a sign that at least part of the electron's mass is due to interaction with its self-field. By 1902, probably under the influence of Abraham, he was claiming that his data showed that the entire mass of the electron was of electromagnetic origin (Miller, 1981, p. 51). Kaufmann continued to find agreement with the predictions based on Abraham's electron model, even after his move to Bonn in 1903 ended the close proximity between theorist and experimentalist.

By 1905, measurements of the velocity dependence of the mass of the electron were supposed to help decide between two different programs in physics, Abraham's electromagnetic program on the one hand and Lorentz's electron theory and Einstein's relativity theory on the other. The theories of Lorentz and Einstein were conflated in this context, not only because they led to the same formulae for the velocity dependence of mass, but also because the main concern of both Lorentz and Einstein was that their theories do justice to the observed relativity of uniform motion, whereas Abraham's main concern was to stay true to his electromagnetic vision. As no single theory met both objectives, a choice had to be made between them. Planck reflected on this predicament in the discussion following a talk he gave on Kaufmann's latest results at the Naturforscherversammlung of 1906. He contrasted Abraham's electrodynamical postulate with the relativity postulate of what was then commonly called the Lorentz-Einstein theory, and stated his preference for the latter. Most of the physicists assembled in Stuttgart considered this the conservative option, as evidenced by the fact, recorded in the published transcript of the discussion, that Sommerfeld's quick retort to Planck's musings brought the house down: "I suspect that the gentlemen under forty will prefer the electrodynamical postulate, while those over forty will prefer the mechanical-relativistic postulate" (Planck, 1906b, p. 761). At 38, Sommerfeld was ten years Planck's junior. Einstein was not in attendance in Stuttgart and it appears that Sommerfeld had not yet read (Einstein, 1905a) when he made this quip (Janssen and Mecklenburg, 2007, p. 113).

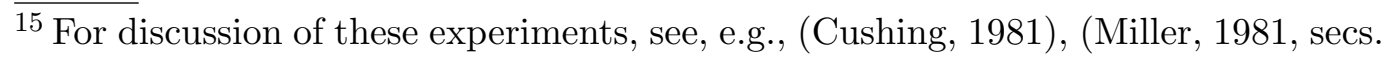
1.11-1.12, 7.4, and 12.4), and (Hon, 1995). 


\subsection{The velocity dependence of electron mass and the electron model of Lorentz and Poincaré}

In his last major paper on the electrodynamics of moving bodies before Einstein's (1905a) celebrated paper on the topic, Lorentz (1904b) systematically elaborated the theory he had outlined in the final section of (Lorentz, 1899). Following Abraham's lead, Lorentz (1904b, sec. 9) proposed a purely electromagnetic electron model of his own. In this model, the electron is a small spherical surface charge distribution, which undergoes a microscopic version of the Lorentz-FitzGerald contraction when it is set in motion with respect to the ether. If the undetermined factor $l$ in Eqs. (14)-(15) is set equal to 1, the electromagnetic momentum of this contractile electron gives formulae for transverse and longitudinal mass of exactly the same form as the ones Lorentz had found in 1899. Lorentz thus killed two birds with one stone. He produced a concrete model of the electron with the velocity dependence needed to help render ether drift invisible and he fixed the value of $l$ (Janssen and Mecklenburg, 2007, sec. 4).

Using Lorentz's theorem of corresponding states, one finds that the energy of the self-field of Lorentz's contractile electron moving through the ether with velocity $\mathbf{v}$ is given by:

$$
U_{\mathrm{EM}}=l\left(\frac{4 \gamma}{3}-\frac{1}{3 \gamma}\right) U_{\mathrm{EM}}^{\prime},
$$

where $U_{\mathrm{EM}}^{\prime}$ is the energy of the self-field of the electron at rest in the ether. Its momentum is similarly given by:

$$
\mathbf{P}_{\mathrm{EM}}=\frac{4}{3} \gamma l\left(\frac{U_{\mathrm{EM}}^{\prime}}{c^{2}}\right) \mathbf{v} .
$$

Inserting this expression for $\mathbf{P}_{\mathrm{EM}}$ into Eqs. (23) and (24) for transverse and longitudinal mass, one arrives at:

$$
m_{\perp}=\frac{P_{\mathrm{EM}}}{v}=l \gamma m^{\prime}, \quad m_{/ /}=\frac{d P_{\mathrm{EM}}}{d v}=\left(l \gamma^{3}+\gamma v \frac{d l}{d v}\right) m^{\prime},
$$

with

$$
m^{\prime}=\frac{4}{3} \frac{U_{\mathrm{EM}}^{\prime}}{c^{2}} .
$$

For $l=1$ these relations have the exact same form as those in Eq. (20): $m_{\perp}=\gamma m^{\prime}$ and $m_{/ /}=\gamma^{3} m^{\prime}$. As soon as $d l / d v \neq 0$, the formula for $m_{/ /}$in Eq. (28) gets more complicated. This then provided Lorentz (1904b, p. 27) with the dynamical argument alluded to in sec. 3.1 for setting $l=1$.

To modern eyes, the factor $4 / 3$ in the mass-energy relation (29) immediately 
makes this model look suspicious, but Lorentz proposed it the year before Einstein (1905b) introduced $E=m c^{2}$. Abraham (1905, p. 204), however, soon spotted this ' $4 / 3$ '-problem, albeit in a different guise: in Lorentz's model energy and momentum give different expressions for the moving electron's longitudinal mass: $(1 / v)\left(d U_{\mathrm{EM}} / d v\right) \neq d P_{\mathrm{EM}} / d v$. This problem is related to that of the stability of Lorentz's contractile electron. What prevents the Coulomb forces from blowing up the surface charge distribution constituting Lorentz's electron?

In 1906, Poincaré solved the stability problem and the ambiguity problem in one fell swoop, albeit at the price of compromising the electromagnetic purity of Lorentz's model. What stabilizes Lorentz's electron, Poincaré (1906) suggested, is a kind of suction by the ether inside of it. This ether suction came to be known as Poincaré pressure or Poincaré stress(es). This stabilizing mechanism also removes the ambiguity in the electron's longitudinal mass. Poincaré assumed that the ether exerts a negative pressure of $-\frac{1}{3}\left(U_{\mathrm{EM}}^{\prime} / V^{\prime}\right)$ on the electron, where $V^{\prime}$ is the volume of the electron at rest (Janssen and Mecklenburg, 2007, sec. 5). The energy associated with this is minus the product of pressure and volume. At rest, $U_{\text {pres }}^{\prime}=\frac{1}{3} U_{\mathrm{EM}}^{\prime}$ and the total energy is:

$$
U_{\mathrm{tot}}^{\prime}=U_{\mathrm{EM}}^{\prime}+U_{\mathrm{pres}}^{\prime}=\frac{4}{3} U_{\mathrm{EM}}^{\prime} .
$$

When the electron is moving with velocity $v$, its volume is $V=V^{\prime} / \gamma$ and $U_{\text {pres }}=U_{\mathrm{EM}}^{\prime} / 3 \gamma$. This is just the opposite of the second term in the expression for $U_{\mathrm{EM}}$ in Eq. (26) for $l=1$. So the total energy of a moving electron is just the first term in this expression:

$$
U_{\mathrm{tot}}=U_{\mathrm{EM}}+U_{\mathrm{pres}}=\frac{4 \gamma}{3} U_{\mathrm{EM}}^{\prime}=\gamma U_{\mathrm{tot}}^{\prime} .
$$

The Poincaré pressure does not affect the electron's momentum. So $\mathbf{P}_{\text {tot }}$ is equal to $\mathbf{P}_{\mathrm{EM}}$ in Eq. (27) for $l=1$ and can be rewritten as:

$$
\mathbf{P}_{\mathrm{tot}}=\gamma\left(\frac{U_{\mathrm{tot}}^{\prime}}{c^{2}}\right) \mathbf{v}=\gamma m^{\prime} \mathbf{v}
$$

with $m^{\prime} \equiv U_{\text {tot }}^{\prime} / c^{2}$. The puzzling factor $4 / 3$ in the mass-energy relation (29) has disappeared.

When Poincaré pressure is taken into account, the expression for the longitudinal mass derived from the electron's electromagnetic energy agrees with the expression derived from its electromagnetic momentum. Substituting the expressions for $U_{\text {tot }}$ and $P_{\text {tot }}$ in Eqs. (31)-(32) for $U_{\mathrm{EM}}$ and $P_{\mathrm{EM}}$ in Eqs. (23)-(25), one finds:

$$
m_{/ /}=\frac{d P_{\mathrm{tot}}}{d v}=\frac{1}{v} \frac{d U_{\mathrm{tot}}}{d v}=\gamma^{3} m^{\prime}, \quad m_{\perp}=\frac{P_{\mathrm{tot}}}{v}=\gamma m^{\prime}
$$


Lorentz and Poincaré had thus produced a model of the electron with exactly the velocity dependence of mass necessary to account for the absence of signs of ether drift. I suppose they can be forgiven for interpreting this as strong evidence that electrons really are contractile spherical surface charge distributions held together by ether suction.

Unfortunately, Kaufmann's data still seemed to favor the formula for the velocity dependence of the electron's transverse mass based on Abraham's model of a purely electromagnetic rigid electron. At the annual Naturforscherversammlung in Cologne in 1908, however, Bucherer reported results of similar experiments favoring what by then was being referred to as the LorentzEinstein formula. Even though special relativity is completely agnostic about nature and structure of the electron, it gives the same formula for the transverse mass of a moving electron as Lorentz's model. With Bucherer's results the experimental tide began to turn in favor of the Lorentz-Einstein formula. By 1915, additional experiments by Schäfer, Neumann, Guye, Lavanchy, Hupka, and others had produced a broad consensus that the data agreed with the Lorentz-Einstein formula and not with Abraham's.

Reanalysis of the data decades later showed that none of these experiments were accurate enough to distinguish between the different predictions for the velocity dependence of the electron mass. Abraham, Lorentz, and Einstein all paid lip service to the importance of these experiments, especially when the data seemed to favor their own theories (see, e.g., Einstein, 1917, p. 51), but it is not clear how seriously they really took them. I suspect that it was not so much the experimental data but theoretical arguments that eventually led to the acceptance of the Lorentz-Einstein formula and eliminated Abraham's model from serious contention. In the discussion following Bucherer's presentation of his data in 1908, for instance, Minkowski ridiculed his young Göttingen colleague Abraham for assuming space-time to be Newtonian in pursuing his electromagnetic program. "Approaching Maxwell's equation with the concept of a rigid electron," he sneered, "seems to me the same thing as going to a concert with cotton in your ears" (quoted from Miller, 1981, p. 330). It was at this same congress in Cologne that Minkowski (1909) gave his now famous lecture introducing the relativistic space-time named after him. By 1915, even proponents of the electromagnetic program, such as Mie, accepted that spacetime is Minkowskian rather than Newtonian and is no place for Abraham's rigid electron. 


\subsection{The velocity dependence of mass as a generic feature of relativistic sys- tems}

The main point of measuring the velocity dependence of electron mass in the first two decades of the 20th century was that it seemed to provide information about the nature and structure of the electron. It was only gradually realized that this was an illusion. The illusion becomes particularly transparent if the electron model of Lorentz and Poincaré is analyzed in terms of relativistic continuum mechanics.

This new framework for a physics of fields rather than particles grew out of the electromagnetic program's new "electromagnetic mechanics," to use Abraham's (1903, p. 109) own phrase. Although Abraham no doubt would have disagreed, one can argue that the development of this framework compensated for the ultimate failure of the program. Minkowski (1908) put Abraham's approach on a solid relativistic basis. In sec. 3.5, I shall argue that Minkowski (1909) also deserves to share the credit with Planck (1908) for freeing the approach from its roots in electrodynamics and recognizing its broader applicability. The new mechanics outlined by Planck was completed by his former student and assistant Laue (1911a,b). Laue, a Privatdozent in Munich at the time, could also build on the elaboration of Minkowski's four-dimensional formalism by Sommerfeld (1910a,b), Laue's senior colleague in Munich. In Laue's relativistic continuum mechanics, the (stress-)energy-momentum tensor for spatially extended systems takes center stage and the vanishing of the four-divergence of the sum of the energy-momentum tensors for all components of a closed system replaces Newton's second law as the fundamental law of dynamics. The famous review article on relativity by Sommerfeld's young student Pauli (1921) contains what to this day remains one of the best expositions of relativistic continuum mechanics. By the time Pauli wrote his article, however, this joint effort of Abraham, Lorentz, Einstein, Minkowski, Planck, Sommerfeld, Laue, and others had already been overshadowed by the development of general relativity and, more importantly, the old quantum theory, which, through Bohr's correspondence principle, gave the old Newtonian particle mechanics a new lease on life.

Special relativity turns the energy and the three components of momentum into the four components of four-momentum:

$$
P^{\mu}=\left(\frac{U}{c}, \mathbf{P}\right) .
$$

For a spatially extended system, such as an electromagnetic field, the fourmomentum is constructed out of the energy-momentum tensor $T^{\mu \nu}$ for the system. This tensor combines the energy density $\left(T^{00}\right)$, the energy flow density $\left(c T^{0 i}\right)$, the momentum density $\left(T^{i 0} / c\right)$, and the stresses or momentum flow 
densities $\left(T^{i j}\right) .{ }^{16}$ Under the standard definition, the components of the fourmomentum of a system described by $T^{\mu \nu}$ are the space integrals:

$$
P^{\mu} \equiv \frac{1}{c} \int T^{\mu 0} d^{3} x
$$

Under this definition, as we shall see below, only the four-momentum of a closed system, i.e., a system for which $\partial_{\nu} T_{\text {tot }}^{\mu \nu}=0,{ }^{17}$ transforms as a four-vector under Lorentz transformations. The Lorentz-Poincaré electron-its electromagnetic field and its Poincaré pressure - is an example of a closed system.

Suppose the four-momentum of some closed system at rest is:

$$
P^{\prime \mu}=\left(U^{\prime} / c, 0,0,0\right)
$$

For a Lorentz boost in the $x$-direction with velocity $v$, the transformation matrix $\Lambda_{\nu}^{\mu}$ is given by (cf. Eq. (14) for $l=1$ ):

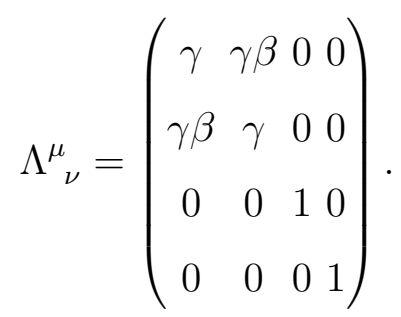

The four-momentum of the closed system moving at velocity $\mathbf{v}=(v, 0,0)$ is:

$$
P^{\mu}=\Lambda_{\nu}^{\mu} P^{\prime \nu}=\left(\frac{\gamma U^{\prime}}{c}, \gamma \frac{U^{\prime}}{c^{2}} \mathbf{v}\right)=\left(\gamma m^{\prime} c, \gamma m^{\prime} \mathbf{v}\right),
$$

with $m^{\prime}=U^{\prime} / c^{2}$. Substituting $U=\gamma m^{\prime} c^{2}$ and $P=\gamma m^{\prime} v$ for $U_{\mathrm{EM}}$ and $P_{\mathrm{EM}}$ in Abraham's Eqs. (23)-(25), one recovers the relations which were first found by Lorentz (1899) (for $l=1$ ) and satisfied by the concrete electron model proposed by Lorentz (1904b) and fixed up by Poincaré (1906):

$$
m_{/ /}=\frac{d P}{d v}=\frac{1}{v} \frac{d U}{d v}=\gamma^{3} m^{\prime}, \quad m_{\perp}=\frac{P}{v}=\gamma m^{\prime} .
$$

This derivation of these relations shows how generic they are. They give the velocity dependence of the mass of any closed system described by Lorentzinvariant laws. Other than that, they reveal nothing about the system. It is because the electron model of Lorentz and Poincaré is closed and Lorentz

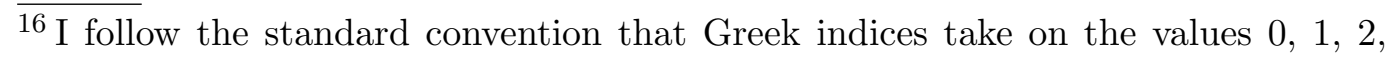
and 3 , corresponding to $c t, x, y$, and $z$, while Latin indices only take on the values 1,2 , and 3 .

${ }^{17}$ As usual, identical upper and lower indices are summed over; $\partial_{\nu}$ is shorthand for $\partial / \partial x^{\nu}$ 
invariant, that their contractile electron obeys these relations; it is because Abraham's model is not Lorentz invariant, that his rigid electron does not.

That the mass of a system obeys the relations in Eq. (39) does not even guarantee that the system is closed. There is an alternative definition of the four-momentum of spatially extended systems, which yields a four-vector regardless of whether the system is open or closed. And, as should be clear from Eqs. (36)-(39), any four-momentum that transforms as a four-vector under Lorentz transformations leads to the same velocity dependence of mass. The freedom one has in defining the four-momentum of spatially extended systems in special relativity will play a central role in the analysis of the Trouton-Noble experiment in the next section, which makes it necessary to look into this issue in some detail.

Both the standard and the alternative definition can be seen as special cases of the following general definition:

$$
P_{\Sigma\left(n^{\rho}, \tau\right)}^{\mu} \equiv \frac{1}{c} \int \delta\left(\eta_{\alpha \beta} x^{\alpha} n^{\beta}-\tau\right) T^{\mu \nu} n_{\nu} d^{4} x
$$

In this equation, $\eta_{\mu \nu}$ is the standard Minkowski metric $\operatorname{diag}(1,-1,-1,-1)$; $\delta(x-a)$ is the Dirac delta function, defined through $\int \delta(x-a) f(x) d x=f(a)$; and $\Sigma\left(n^{\rho}, \tau\right)$ is a spacelike hyperplane fixed by $(i)$ the timelike unit vector $n^{\rho}$ orthogonal to it (in the sense of the standard Minkowski inner product) and (ii) its orthogonal distance $\tau$ to the origin $O$ of the $x^{\mu}$-coordinate system ( $\tau$ is the proper length of a timelike line segment in the direction of $n^{\rho}$ that begins in $O$ and ends on the hyperplane). The delta function picks out all points and only those points that lie on this hyperplane and thus turns the four-dimensional integral into a three-dimensional one.

One arrives at different definitions of the four-momentum of spatially extended systems by adopting different conventions for choosing a family of hyperplanes $\left\{\Sigma\left(n^{\rho}, \tau\right)\right\}$ in Eq. (40). Fig. 2 illustrates two of the three basic options. The third is not to privilege any hyperplanes and to accept that the four-momentum of a spatially extended system is a hyperplane-dependent quantity (Fleming, 2000). Fig. 2 shows a two-dimensional Minkowski spacetime with two sets of space and time axes, one for the frame of some observer, one for the rest frame of some spatially extended object, such as the LorentzPoincaré electron. The shaded region represents the world sheet of this object. The standard convention is to pick hyperplanes for which $n^{\rho}$ is in the time direction in the frame of the observer. This vector, $n_{\mathrm{obs}}^{\rho}$, is different in each frame but it has the same components $(1,0,0,0)$ in all of them. Integrals of $T^{\mu \nu} n_{\nu}$ over $\Sigma\left(n_{\text {obs }}^{\rho}, \tau_{\text {obs }}\right)$ will consequently be ordinary space integrals of $T^{\mu 0}$ in all frames. The general definition (40) thus reduces to the standard non-covariant 


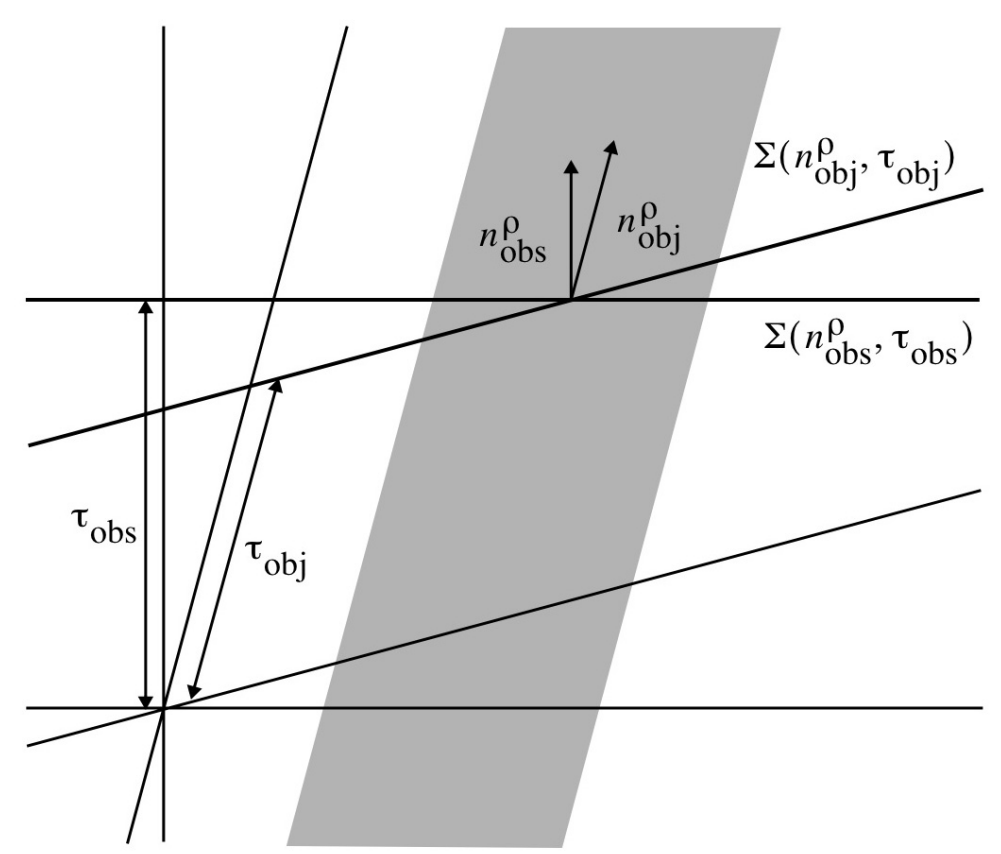

Fig. 2. Different conventions for picking hyperplanes in defining the four-momentum of spatially extended systems

definition (35):

$$
P_{\mathrm{stan}}^{\mu} \equiv P_{\Sigma\left(n_{\mathrm{obs}}^{\rho}, \tau_{\mathrm{obs}}\right)}^{\mu}=\frac{1}{c} \int T^{\mu 0} d^{3} x
$$

The alternative convention is to pick hyperplanes for which $n^{\rho}$ is in the time direction in the rest frame of the object under consideration. This vector, $n_{\mathrm{obj}}^{\rho}$, is the same in every frame even though it will have different components in each of them. It is not always possible to define the rest frame uniquely, but this is not a problem for systems that are static except for a constant velocity of the system as a whole, such as the Lorentz-Poincaré electron or the capacitor in the Trouton-Noble experiment examined in the next section. With this alternative convention, the general definition (40) turns into a covariant definition:

$$
P_{\mathrm{cov}}^{\mu} \equiv P_{\Sigma\left(n_{\mathrm{obj}}^{\rho}, \tau_{\mathrm{obj}}\right)}^{\mu} .
$$

This alternative definition was first proposed by Fermi (1921, 1922), forgotten, rediscovered several times, and finally broadly disseminated by Rohrlich (1960, 1965). Some of the discussion in the literature over which of these two definitions is preferable may suggest that one is right and the other is wrong. ${ }^{18}$ Since the difference between the two is a convention about choosing a family of hyperplanes, this cannot be the issue. At most, one might want to argue that one definition is more convenient, more elegant, or more natural than the other.

\footnotetext{
${ }^{18}$ See, in particular, the 'Boyer-Rohrlich controversy' discussed in (Campos and Jiménez, 1986). I am grateful to Nico Giulini for drawing my attention to this paper.
} 
The two definitions coincide in the rest frame, but, in general, give different results in frames in which the system under consideration is moving. Under the covariant definition (42), the four-momentum of a moving system is always the Lorentz transform of the four-momentum in its rest frame: $P_{\mathrm{cov}}^{\mu}=\Lambda^{\mu}{ }_{\nu} P_{\mathrm{cov}}^{\prime \nu}$. Under the standard definition (41), this will, in general, not be case: $P_{\text {stan }}^{\mu} \neq$ $\Lambda_{\nu}^{\mu}{ }_{\nu} P_{\text {stan }}^{\prime \nu}$. It is easy to see why. $P_{\text {stan }}^{\prime \mu}\left(=P_{\text {cov }}^{\prime \mu}\right)$ is an integral over a hyperplane of simultaneity in the system's rest frame. Its Lorentz transform, $\Lambda_{\nu}^{\mu} P_{\text {stan }}^{\prime \nu}(=$ $P_{\text {cov }}^{\mu}$, remains an integral over that same hyperplane, which is not a hyperplane of simultaneity in the new frame. However, if the system is closed, i.e., if $\partial_{\nu} T^{\mu \nu}=0$, the results of integrating over different hyperplanes are always the same (barring pathological behavior as the spatial coordinates go to infinity). This follows from a straightforward application of the obvious generalization of Gauss's theorem from three to four dimensions. ${ }^{19}$ For closed systems, fourmomentum therefore does transform as a four-vector, even under the standard definition. In summary: for closed systems, definitions (41) and (42) coincide in all frames; for open systems, they only coincide in the rest frame.

It will be instructive to relate the four-momentum, under the standard definition, of the electromagnetic field and the Poincare pressure of a moving Lorentz-Poincaré electron to the four-momentum of field and pressure of the same electron at rest. Unprimed quantities refer to the former situation, primed ones to the latter. In pre-relativistic terms, motion and rest are to be understood here with respect to a frame at rest in the ether. In special relativity, it can be with respect to any inertial frame. The transition from primed to unprimed quantities can be interpreted either as an active or as a passive transformation. So far, the discussion has been in terms of active transformations: the comparison has been between two different situations, that of an electron at rest (primed) and that of an electron in motion (unprimed) in the same frame (a frame at rest in the ether). But a passive interpretation is also possible: in that case the comparison is between two different perspectives on the same situation, an electron viewed from its rest frame (primed) and from a frame in which it is moving (unprimed). For continuity with the ether-theoretic discussion so far, it is best to proceed in terms of active transformations.

The energy-momentum tensor for the field of a static charge distribution at rest is:

$$
T_{\mathrm{EM}}^{\prime \mu \nu}=\left(\begin{array}{cc}
u_{\mathrm{EM}}^{\prime} & 0 \\
0 & -T_{\mathrm{Max}}^{\prime i j}
\end{array}\right),
$$

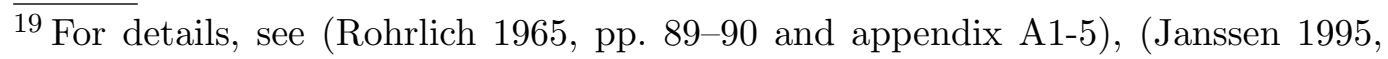
sec. 2.1.3), or (Teukolsky 1996, p. 1105). 
where $T_{\text {Max }}^{\prime i j}$ is the Maxwell stress tensor, which is this case is given by:

$$
T_{\mathrm{Max}}^{\prime i j}=\varepsilon_{0} E^{\prime i} E^{\prime j}-\delta^{i j} u_{\mathrm{EM}}^{\prime}
$$

(with $\delta^{i j} \equiv \operatorname{diag}(1,1,1)$ the Kronecker delta). The energy-momentum tensor for the Poincaré pressure on the electron at rest is (Janssen and Mecklenburg, 2007, sec. 6):

$$
T_{\text {pres }}^{\prime \mu \nu}=\frac{1}{3}\left(\frac{U_{\mathrm{EM}}^{\prime}}{V^{\prime}}\right) \eta^{\mu \nu} \vartheta\left(R-r^{\prime}\right),
$$

where $R$ is the radius of the electron at rest, $r^{\prime} \equiv \sqrt{x^{\prime 2}+y^{\prime 2}+z^{\prime 2}}$, and $\vartheta(x)$ is a step-function that ensures that the pressure is constant inside the electron and vanishes outside $(\vartheta(x)=0$ for $x<0$ and $\vartheta(x)=1$ for $x \geq 0)$. Field and pressure together form a closed system:

$$
\partial_{\nu}^{\prime}\left(T_{\mathrm{EM}}^{\mu \nu}+T_{\mathrm{pres}}^{\prime \mu \nu}\right)=0
$$

This can readily be verified for an electron at rest. Since Eq. (46) is a tensor equation, it is also true for an electron in uniform motion. From the point of view of relativistic continuum mechanics, Poincaré pressure is nothing but the piece that needs to be added to Lorentz's purely electromagnetic electron to turn it into a closed system. This is physically much more transparent than the way in which Poincaré originally introduced this quantity. Since the system is static, the $(\nu=0)$-component of Eq. (46) vanishes no matter what function of $\mathbf{x}^{\prime}$ is chosen for $T_{\mathrm{pres}}^{\prime 00}$. So Poincaré's solution to the ambiguity problem and the stability problem of Lorentz's purely electromagnetic electron is not unique (Janssen and Mecklenburg, 2007, p. 120, note 52).

Using the standard definition (41) of four-momentum along with expression (43) for $T_{\mathrm{EM}}^{\prime \mu \nu}$ and the standard rules for the transformation of this tensor under the Lorentz boost (37), one finds that the four-momentum of the electromagnetic field of the moving electron is given by:

$$
P_{\mathrm{EM}}^{\mu}=\frac{1}{c} \int \Lambda_{\rho}^{\mu} \Lambda_{\sigma}^{0} T_{\mathrm{EM}}^{\prime \rho \sigma} d^{3} x=\left(\left(\frac{4 \gamma}{3}-\frac{1}{3 \gamma}\right) \frac{U_{\mathrm{EM}}^{\prime}}{c}, \frac{4}{3} \gamma\left(\frac{U_{\mathrm{EM}}^{\prime}}{c^{2}}\right) \mathbf{v}\right) .
$$

Because the system is static, the integral over a hyperplane of simultaneity in the unprimed frame can be replaced by an integral over a hyperplane of simultaneity in the primed frame. One can thus replace the volume element $d^{3} x$ by $d^{3} x^{\prime} / \gamma$ and use that $\int \frac{1}{2} \varepsilon_{0} E_{i}^{\prime 2} d^{3} x^{\prime}=\frac{1}{3} \int u_{\mathrm{EM}}^{\prime} d^{3} x^{\prime}=\frac{1}{3} U_{\mathrm{EM}}^{\prime}$. This was done in the last step in Eq. (47). This equation combines Eqs. (26) and (27) (both for $l=1$ ) for $U_{\mathrm{EM}}$ and $\mathbf{P}_{\mathrm{EM}}$. For the four-momentum of the Poincaré pressure in the moving electron, one similarly finds:

$$
P_{\text {pres }}^{\mu}=\frac{1}{c} \int \Lambda_{\rho}^{\mu} \Lambda_{\sigma}^{0} T_{\text {pres }}^{\prime \rho \sigma} d^{3} x=\left(\frac{1}{3 \gamma} \frac{U_{\mathrm{EM}}^{\prime}}{c}, 0,0,0\right) .
$$


Adding these two four-momenta and setting $U_{\text {tot }}^{\prime}=\frac{4}{3} U_{\mathrm{EM}}^{\prime}$, one recovers Eqs. (31) and (32) for the total energy and momentum of the moving electron:

$$
P_{\mathrm{tot}}^{\mu}=P_{\mathrm{EM}}^{\mu}+P_{\mathrm{pres}}^{\mu}=\left(\gamma \frac{U_{\mathrm{tot}}^{\prime}}{c}, \gamma \frac{U_{\mathrm{tot}}^{\prime}}{c^{2}} \mathbf{v}\right)
$$

So, under the standard definition (41), the total four-momentum of the electron, a closed system, transforms as a four-vector under Lorentz transformations, but the four-momenta of its open subsystems (electromagnetic field and Poincaré pressure) do not. Under the alternative definition (42), the fourmomentum of the system as a whole and the four-momenta of its two open subsystems considered separately all transform as four-vectors. The deviations from $\Lambda^{\mu}{ }_{\nu} P_{\mathrm{EM}}^{\prime \nu}$ and $\Lambda^{\mu}{ }_{\nu} P_{\mathrm{pres}}^{\prime \nu}$ in Eqs. (47) and (48) for $P_{\mathrm{EM}}^{\mu}$ and $P_{\text {pres }}^{\mu}$ - the puzzling factors $4 / 3$ and $1 / 3$ in particular-are artifacts of the non-covariant standard definition of four-momentum.

\subsection{Drawing the line between kinematics and dynamics in accounting for the velocity dependence of mass}

Lorentz (1899) showed that the mass of particles interacting with electromagnetic fields in his theory has to depend on velocity in a particular way to help account for the absence of signs of ether drift. If the undetermined scale factor $l$ is set equal to 1, this is the same velocity dependence that Einstein (1905a) and Planck (1906a) showed is required by the postulates of special relativity. The relativistic derivation is mathematically equivalent to Lorentz's derivation for $l=1$. Both derivations show how mass, defined as the ratio of force and acceleration, transforms in a world governed by Lorentz-invariant laws in which Newton's second law holds in a particle's rest frame. Lorentz, understandably, was not impressed with the relativistic reincarnation of his 1899 derivation. For him, this derivation was only a first step. It showed that his theory required a non-Newtonian concept of inertia. The next step was to explain how such inertia could arise. To this end, Lorentz (1904b), following Abraham (1902a,b) and with help from Poincaré (1906), developed a concrete electromagnetic model of the electron with exactly the required velocity dependence of mass. Referring at least in part to the transformation law for mass, he wrote in the final section of a book based on lectures at Columbia University in 1906: "Einstein simply postulates what we have deduced, with some difficulty and not altogether satisfactorily, from the fundamental equations of the electromagnetic field" (Lorentz, 1915, pp. 229-230). ${ }^{20}$

20 The element that Lorentz explicitly identifies in this passage as something Einstein "deduces ... from the relativity principle itself" is the relativistic transformation law for charge density needed to complete the proof of the Lorentz invariance of Maxwell's equations in for arbitrary charge distributions, which had eluded Lorentz 
This remark is indicative of Lorentz's general attitude toward special relativity. In an article in the London Times, Einstein (1919) later declared special relativity to be a "principle theory," like thermodynamics, rather than a "constructive theory," like the kinetic theory of gases. This distinction can be used to capture the way in which Lorentz saw the difference between Einstein's theory and his own. I use the principle-constructive distinction in the following sense (I shall have more to say below about Einstein's own use of it). ${ }^{21}$ To set up a principle theory for a certain class of phenomena, one raises some observed regularities about these phenomena to the level of postulates. The only thing a principle theory says about the underlying reality is that all phenomena it gives rise to are in accordance with the postulates. To set up a constructive theory covering the same class of phenomena, one posits a specific model for the reality behind them. A principle theory for these phenomena will constrain such modeling but offers no grounds for preferring one model satisfying the constraints over another. Lorentz looked upon his own theory as providing a concrete model of a world in accordance with the postulates of special relativity. In this sense, Lorentz's constructive theory thus complements rather than contradicts Einstein's principle theory.

Special relativity, however, is not a principle theory in this very strict sense and Einstein never intended it to be one. For one thing, he clearly wanted to reject any constructive theory that posits an ether, even one as bare bones as Lorentz's, or that singles out the inertial frames at rest in it, even if it is impossible to pick out such preferred frames empirically. Even if it were stripped of these two objectionable elements, however, Lorentz's theory would still be at odds with Einstein's theory. The principle-constructive distinction is a red herring in the end. By focusing on it, Lorentz missed a much more important difference between his own theory and Einstein's, namely the difference between kinematical and dynamical accounts of a whole class of phenomena. It was for a reason that Einstein called the first half of his 1905 paper "The kinematical part."

According to special relativity, as shown in this section, the velocity dependence of mass has nothing to do with the specifics of the dynamics of electromagnetism, ether pressure, or what have you, but is a generic property of closed systems in a world in accordance with the postulates of the theory. The velocity dependence of mass is thus kinematical in the broad sense of being independent of the details of the dynamics. I shall argue below that this is because it is also kinematical in the narrow sense of exemplifying default spatio-temporal behavior. For now I focus on the claim that it is kinematical in

(Janssen, 1995, secs. 3.5.6 and 4.3.2).

${ }^{21}$ See (Balashov and Janssen, 2003, sec. 4). Brown and Pooley (2006, p. 73) praise our characterization of the distinction but prefer a less rigid one themselves (see also Brown, 2005, pp. 89-90). 
the broad sense. Detailed dynamical considerations, i.e., considerations specific to the system at hand, do come into play if one wants to account for the origin of the rest mass $m^{\prime}$ of a particular particle. Given $E=m c^{2}$, this boils down to the calculation of any and all contributions to the particle's rest energy with the help of theories that, as required by the postulates of special relativity, are all Lorentz invariant. Another option is simply to accept the empirical value of a particle's rest mass as a brute fact. Either way, assuming that a particle is a prototypical closed system, ${ }^{22}$ one ends up with the four-momentum $P^{\prime \mu}=\left(m^{\prime} c, 0,0,0\right)$ in the particle's rest frame. The four-momentum of the same particle moving with velocity $\mathbf{v}$ will be $P^{\mu}=\Lambda_{\nu}^{\mu} P^{\prime \nu}=\left(\gamma m^{\prime} c, \gamma m^{\prime} \mathbf{v}\right)$. The relations $m_{/ /}=\gamma^{3} m^{\prime}$ and $m_{\perp}=\gamma m^{\prime}$ are direct consequences of this transformation law (see Eq. (39)). These relations are therefore kinematical in the broad sense.

Pais (1982) also used the term 'kinematical' in this broad sense when he wrote in his Einstein biography: "Special relativity killed the classical dream of using the energy-momentum-velocity relations as a means of probing the dynamical origins of [the electron mass]. The relations are purely kinematical" (p. 159). By 1920, it had become clear to many physicists that this 'classical dream' had led them on a wild goose chase. In his popular book on relativity, Einstein (1917) drew a parallel between the velocity dependence of mass of the electron - or the "law of motion" (p. 50) as he referred to it (see Eq. (19) for $l=1$ ) - and the Fresnel drag coefficient discussed in sec. 2:

The theory of relativity leads to the same law of motion, without requiring any special hypothesis whatsoever as to the structure and the behavior of the electron. We arrived at a similar conclusion ... with the experiment of Fizeau, the result of which is foretold by the theory of relativity without the necessity of drawing on hypotheses as to the physical nature of the liquid (Einstein, 1917, p. 51).

Lorentz conceded the point in lectures at Caltech in 1922. Compare the following passage to the one quoted above from his lectures at Columbia in 1906:

The formula for momentum was found by a theory in which it was supposed that in the case of the electron the momentum is determined wholly by that of the electromagnetic field ... This meant that the whole mass of an electron was supposed to be of electromagnetic nature. Then, when the formula for momentum was verified by experiment, it was thought at first that it was thereby proved that electrons have no "material mass." Now we can no longer say this. Indeed, the formula for momentum is a general consequence of the principle of relativity, and a verification of that formula

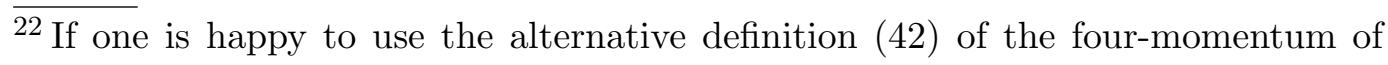
spatially extended systems, one does not even have to assume that much. 
is a verification of the principle and tells us nothing about the nature of mass or of the structure of the electron (Lorentz, 1927, p. 125).

In 1906, Lorentz, hinting at his own dynamical explanation of the velocity dependence of mass, suggested that Einstein had come up short by not providing one. In 1922, Lorentz recognized that he himself had overshot the mark. Pauli addressed the same issue in his review article on special relativity:

It constituted a definite progress that Lorentz's law of the variability of mass could be derived from the theory of relativity without making any specific assumptions on the electron shape or charge distribution. Also nothing need be assumed about the nature of the mass (Pauli, 1921, pp. 82-83).

Given that he clearly realized that the velocity dependence of mass is kinematical (in the broad sense), it may come as a surprise that Pauli, earlier in his review article, called for a dynamical explanation of length contraction. In a passage quoted approvingly by Brown (2005, p. 4, p. 118), he wrote:

Should one then completely abandon any attempt to explain the Lorentz contraction atomistically? We think that the answer to this question should be No. The contraction of a measuring rod is not an elementary but a very complicated process. It would not take place except for the covariance with respect to the Lorentz group of the basic equations of electron theory, as well as of those laws, as yet unknown to us, which determine the cohesion of the electron itself (Pauli, 1921, p. 15).

Pauli here conflates solving a difficult problem and appealing to an automatic corollary of its solution. The problem is how to construct a Lorentz-invariant model of a physical system at rest that can serve as a measuring rod. The model would have to be quantum-mechanical as classical theories cannot account for the stability of matter. What calls for a dynamical or "atomistic" explanation, however, is precisely this stability and not length contraction. Once a stable model of a system at rest is in place, Lorentz invariance guarantees that a contracted version of the same system in uniform motion will also be stable. This is true for any Lorentz-invariant model. Length contraction, like the velocity dependence of mass, is thus kinematical, at least in the broad sense.

Why did Pauli recognize this in one case but not in the other? Part of the answer, I suspect, is that Pauli was more careful in the case of mass because it had tripped up several of his elders. The search for a dynamical explanation of the velocity dependence of mass had seduced them into making completely unwarranted inferences about the nature of the electron and the nature of mass in general. As I mentioned in the introduction, it is exactly because explanation is routinely used as a guide to inference that it matters - or should matter - to scientists and not just to philosophers to get their explanatory 
stories straight. Pauli would presumably have been more careful in the case of length contraction as well, had his contemporaries been in the habit of making bold leaps from length contraction to shaky yet Lorentz-invariant theories of the stability of matter.

My diagnosis of the error in Pauli's comments on length contraction also serves as a rebuttal of Brown's (2005) provocative claim that Einstein himself "never fully made the transition from the old dynamics to the new kinematics" (p. vii, p. 114). Brown borrows this formulation from Pais (1982, p. 167), who used it to pass judgement on Lorentz's attitude toward special relativity. To support his claim, Brown (2005, pp. 113-114) quotes several passages from Einstein's writings in which he concedes the need for dynamical models of systems that can be used as rods and clocks. What originally prompted these remarks by Einstein was Weyl's (1918) attempt to unify gravity and electromagnetism. ${ }^{23}$ Unlike the young Pauli, however, Einstein never mistook this for a dynamical explanation of length contraction or time dilation. Those phenomena, to reiterate, are direct consequences of the Lorentz invariance assumed from the get-go in the construction of any acceptable dynamical model of rods or clocks. They are thus purely kinematical, at least in the broad sense.

Length contraction, time dilation, and the velocity dependence of mass are also kinematical in the narrow sense of exemplifying standard spatio-temporal behavior in special relativity. The Lorentz invariance that can be derived from the postulates of special relativity (in conjunction with the assumption that space and time are homogeneous and isotropic) finds its natural interpretation in terms of the geometry of Minkowski space-time. This interpretation amounts to the constructive-theory version of special relativity. It says that the space-time component of any acceptable model of a world in accordance with the postulates is Minkowski space-time. Lorentz's theory was a comprehensive constructive theory of matter and fields in a Newtonian space-time. Special relativity did not replace that theory with one of comparable scope, nor was it ever intended to. Quantum mechanics eventually would. Special relativity only eliminated certain parts of Lorentz's theory, notably the ether and remnants of Newtonian theory. It did this by providing a new interpretation of Lorentz invariance, which had emerged as an important new property of physical laws in Lorentz's theory. In this new interpretation, the property of Lorentz invariance is no longer accidentally shared by all dynamical laws governing systems in Newtonian space and time but reflects the structure of a new relativistic space-time (Janssen, 2002a). There is no need to reify this space-time structure to the point that Lorentz invariance becomes a property of a substantival space-time arena, although Norton (2007) may be right that some reification is unavoidable (cf. my comments in sec. 1.2). Presumably, the

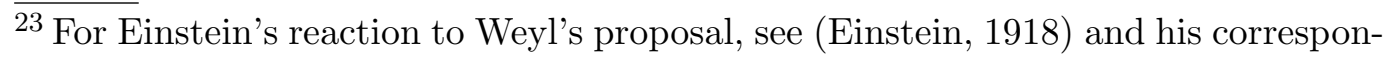
dence with Weyl in 1918 (Einstein, 1987-2006, Vol. 8B). 
laws can still be the bearers of the property of Lorentz invariance. The key point is that this property has nothing to do with electrodynamics per se, nor with any other Lorentz-invariant dynamical theory one might contemplate.

The interpretation of Lorentz invariance defended here is similar to the one offered by Minkowski in the conclusion of "Space and time:"

The validity without exception of the world-postulate, I like to think, is the true nucleus of an electromagnetic image of the world, which ${ }^{24}$ discovered by Lorentz, and further revealed by Einstein, now lies open in the full light of day (Minkowski, 1908, p. 91).

Earlier in his talk, Minkowski had defined the 'world-postulate' (or 'postulate of the absolute world') as: "only the four-dimensional world in space and time is given by phenomena, but ... the projection in space and in time may still be undertaken with a certain degree of freedom" (ibid., p. 83). Following Jon Dorling (private communication), I used to read Minkowski's text as endorsing the view that the Lorentz invariance of Maxwell's equations explains why space-time is Minkowskian (Janssen, 1995, sec. 3.5.7). If Maxwell's equations are replaced by (perhaps yet to be found) quantum-mechanical dynamical equations, this is Brown's view, although he interprets both Minkowski's text and Dorling's exegesis of it differently (Brown, 2005, p. 130). I have come to prefer the exact opposite reading of Minkowski. On Dorling's reading, the "true nucleus" of the electromagnetic program is that Maxwell's equations form the theory of everything including, through their Lorentz invariance, the structure of space and time. On the alternative reading, which was first suggested to me by Leo Corry, ${ }^{25}$ the "true nucleus" is the kernel of truth in a program that otherwise may or may not pan out. Minkowski's point, on this reading, is that the space-time structure reflected in the Lorentz invariance of Maxwell's equations is here to stay, whether or not all of physics can ultimately be reduced to those equations.

On this view - leave aside for the moment whether this was indeed Minkowski's or, for that matter, Einstein's - it is clear that length contraction is purely kinematical in the narrow as well as in the broad sense. That a system can serve as a measuring rod in its rest frame will depend on the details of the dynamics for that system. That this system, when set in motion, undergoes length contraction is guaranteed by the fact that the dynamics is Lorentz invariant, which, in turn, reflects nothing but the space-time structure posited by special relativity understood as a constructive theory. More concretely, a contracting rod simply exhibits the default spatio-temporal behavior encoded in Minkowski space-time. For the velocity dependence of mass, defined as the ratio of force and acceleration, the situation is a little more complicated.

\footnotetext{
${ }^{24}$ In the German original it is unambiguous that "which" refers back to "nucleus." ${ }^{25}$ Private communication. See also (Corry, 1997).
} 
These relations depend not just on the transformation laws for length and time, which suffice to derive the transformation law for acceleration (Eq. (17) for $l=1$ ), but also on the transformation law for force (Eq. (16) for $l=1$ ). In relativistic continuum mechanics, force is a derived quantity, which inherits its transformation properties from the energy-momentum tensor. ${ }^{26}$ Relativistic continuum mechanics is tailored to the structure of Minkowski space-time. The behavior of its general concepts, such as the energy-momentum tensor or the four-momentum, under Lorentz transformations reflect this space-time structure. So even though the transformation law for mass cannot be established without reference to concepts over and above spatio-temporal ones, the velocity dependence of mass is still purely kinematical, even in the narrow sense. $^{27}$

To conclude this section, I want to argue that the principle-constructive distinction not only tripped up Lorentz but Einstein as well. In the process, I shall highlight the important positive heuristic role that special relativity has to play - even if conceived of as a constructive theory - in the ongoing search for acceptable successors to the dynamical theories of Lorentz and Abraham. I already identified its negative heuristic role, which is to prevent the folly of developing dynamical accounts for purely kinematical phenomena.

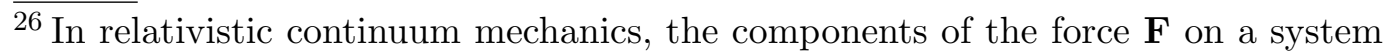
that is static except for the overall velocity $v$ in the $x$-direction is defined as:

$$
F^{i} \equiv-\int \partial_{j} T^{i j} d^{3} x
$$

where $T^{\mu \nu}$ is the energy-momentum tensor for the system. The integrand can be rewritten as $\Lambda^{i}{ }_{k} \partial_{j}^{\prime} T^{\prime k j}$ (where Eq. (37) gives $\operatorname{diag}(\gamma, 1,1)$ for $\Lambda^{i}{ }_{j}$ ); the volume element as $d^{3} x^{\prime} / \gamma$ (cf. the discussion following Eq. (47)). It follows that $\mathbf{F}=\operatorname{diag}(1,1 / \gamma, 1 / \gamma) \mathbf{F}^{\prime}$

${ }^{27}$ Brown (2005, p. 86) states matter-of-factly that this is not a kinematical effect, probably because the velocity dependence of mass involves Lorentz transformation equations over and above those for the space-time coordinates. Here is another example to show that one cannot jump to that conclusion. Consider the electric field of a charge at rest (or the magnetic field of a magnet at rest). In a frame in which the charge (or the magnet) is moving, this same field is a combination of an electric and a magnetic field. This follows directly from the transformation law, $F^{\mu \nu}=\Lambda_{\rho}^{\mu} \Lambda_{\sigma}^{\nu} F^{\prime \rho \sigma}$, for the electromagnetic field strength tensor, which combines the components of $\mathbf{E}$ and $\mathbf{B}$. An active reading of this same transformation law leads to the conclusion that a moving charge (or a moving magnet) produces a combination of an electric and a magnetic field. This is a purely kinematical effect, in the narrow and a fortiori in the broad sense. It has the same status as the "effect" that an electric field in the $x$-direction has components in both the $x$ - and the $y$ direction if the spatial coordinate system and the system under consideration are is rotated with respect to one another. 
As Einstein (1949, p. 53) made clear in a well-known passage in his autobiographical notes, he only resorted to a principle theory in the work that led to special relativity because he could not find a constructive theory to replace Lorentz's comprehensive theory of matter and fields. The principle theory he eventually came up with certainly provided some useful constraints on such a constructive theory, but ultimately it was that constructive theory itself that he was after. In the 1919 Times article in which he introduced the principle-constructive distinction, he expressed a strong preference for constructive theories: "When we say that we have succeeded in understanding a group of natural processes, we invariably mean that a constructive theory has been found which covers the processes in question" (Einstein, 1919, p. 228; quoted in Brown, 2005, p. 113). In a letter of early 1908, he had already expressed this preference in the specific case of special relativity: "The theory of relativity is ultimately as little satisfactory as ... thermodynamics was before Boltzmann had interpreted the entropy as probability." 28

I argued above that Minkowski's lecture "Space and time," delivered later in 1908, did for special relativity, understood strictly as a principle theory, what Boltzmann's interpretation of entropy had done for the second law of thermodynamics. It turned special relativity into a constructive theory by providing the concrete model for the reality behind the phenomena covered by principle theory. Einstein, however, is reported to have denounced Minkowski's work, at least initially, as "superfluous learnedness" (Pais, 1982, p. 152; see also Janssen, 1995, sec. 3.5.7, note 122). To the extent that Minkowski merely formalized what Einstein himself had already recognized in 1905, i.e., the need for a new kinematics characterized by Lorentz invariance rather than Galilean invariance, this is an understandable assessment. Einstein changed his mind about Minkowski's contribution once he recognized its value for the development of general relativity. Yet, in the Times article, flush with the success of general relativity in the wake of the British eclipse expeditions, he still insinuated that a constructive version of special relativity had yet to be found. I claim that Minkowski had already found it more than a decade earlier. If Einstein ever came to appreciate this, he certainly never gave Minkowski credit for it (Brown, 2005, p. 138).

I nonetheless want to maintain, pace Brown, that Einstein's position was no different from the one I am defending in this paper. My textual evidence comes from an oft-quoted letter written the year of his death, but I see no reason to believe that Einstein's stance on this particular issue changed much between 1905 and 1955. Let me emphasize that this is not meant as an argument from authority for my position. That position should be judged on its own merits, regardless of how close it is to Einstein's or anyone else's at one time or another.

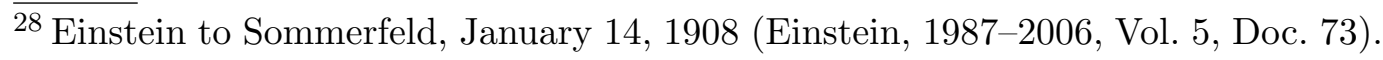


The key observation that reconciles my interpretation of Einstein's position with the well-known passages discussed above is that Einstein's continued demand for a constructive theory was for a new constructive theory of everything, for a worthy successor to Lorentz's discredited comprehensive theory, and not for the constructive theory that would get at the reality behind the phenomena covered by special relativity understood strictly as a principle theory. That theory had already been found. It had identified the reality underlying the relevant phenomena as Minkowski space-time. ${ }^{29}$ This space-time can be thought of in substantival or in relational terms. Special relativity does not decide which systems get to inhabit or, as the relationist might prefer, carry Minkowski space-time. All special relativity has to say about such systems is that their spatio-temporal behavior must obey the rules encoded in Minkowski space-time. This requirement is automatically met if the system is governed by Lorentz-invariant laws. Special relativity thus imposes a kinematical constraint on all dynamical laws (cf. my remarks in sec. 1.2). So, even as a constructive theory, special relativity plays the heuristic role of providing constraints on further theorizing. This heuristic role was the important feature of principle theories for Einstein. He did not introduce the principleconstructive distinction to put labels on already established theories. He was essentially characterizing different strategies for finding new ones - develop a concrete model (the constructive strategy) or find constraints on such models (the principle strategy). This makes it perfectly understandable that Einstein would continue to characterize special relativity as a principle theory long after the corresponding constructive theory had been found.

A letter to his biographer Seelig of February 19, 1955, yields clear evidence of Einstein's views both on what special relativity has to say about the reality behind the various phenomena Lorentz invariance gives rise to (such as length contraction and the velocity dependence of mass) and on the heuristic value of Lorentz invariance in the search for the reality behind all sorts of other phenomena. First, he provided as lucid a formulation of the position defended in this paper as one could hope for. He explicitly identified the "new feature" of special relativity in 1905 as "the realization that the Lorentz transformation transcends its connection with Maxwell's equations and has to do with the nature of space and time in general." He identified its heuristic role as a "further new result:" "Lorentz invariance is a general condition for any physical theory. This was for me of particular importance because I had already found that Maxwell's theory ... could not ... have general validity." 30

\footnotetext{
${ }^{29}$ A similar observation applies to general relativity, which Einstein also characterized as a principle theory in his 1919 article. Presumably, to use his famous metaphor (Einstein, 1954, p. 311), he was calling for a better understanding of the 'wood' of the matter on the right-hand side of his gravitational field equations, not of the 'marble' of the geometry on the left-hand side.

${ }^{30}$ Both passages are quoted and discussed in (Brown, 2005, p. 73), where the recip-
} 


\section{The Trouton-Noble experiment and its delicately balanced torques}

\subsection{Detecting ether drift with capacitors: from looking for impulses (Trouton) to looking for torques (Trouton-Noble)}

The Trouton-Noble experiment grew out of the now largely forgotten Trouton experiment. The object of both experiments was to detect ether drift with capacitors hanging from the ceiling of the laboratory. Because of this family resemblance, the two experiments tend to get conflated, ${ }^{31}$ but they were designed to detect different effects and their negative results likewise call for different explanations. The Trouton experiment was supposed to detect an impulse upon charging a capacitor moving through the ether; its better-known sequel a torque on an already charged moving capacitor.

The story of these experiments begins in Dublin in 1900, when FitzGerald thought of an electrical as opposed to an optical method to detect the earth's presumed motion through the ether. Trouton, his assistant, designed and carried out the actual experiment. When the plates of a capacitor at rest in the ether are connected to the poles of a battery, the battery supplies the energy for the electric field between the plates. What happens when the capacitor is charged in a laboratory moving with the earth through the ether? The charges on the moving plates now constitute two currents, so there will typically be a magnetic as well as an electric field. Where does the energy for this magnetic field come from? FitzGerald, for reasons best known to himself, thought that it could not come from the battery but would have to come instead from the kinetic energy of the capacitor. In that case, a capacitor freely suspended on a wire from the ceiling of the laboratory should, upon being charged, experience a sudden mechanical jolt in the direction of the ether wind. However, as Lorentz (1904b) put it, "Trouton was unable to observe these jerks" (p. 830).

In 1901, before the final results of the experiment were in, FitzGerald unexpectedly died at the early age of 49. Trouton's (1902) report on the experiment he had suggested was included in a collection of his scientific papers edited by Larmor (FitzGerald, 1902). In an editorial note on Trouton's paper, Larmor (1902) argued that the negative result of the experiment was only to be expected. From a relativistic point of view, Larmor's conclusion is obviously correct but the argument that led him to it is not. By modern lights, he failed

ient of the letter is unfortunately misidentified as Born. Miller (1981, Ch. 6, p. 195) fittingly uses the same passage as the motto for the chapter on "[t]he relativistic transformations." On Einstein's path to special relativity and the other results of his annus mirabilis, see (Renn and Rynasiewicz, 2007).

${ }^{31}$ See, e.g., (Warwick, 1995, p. 318; Teukolsky, 1996, p. 1104; Brown, 2007, p. 55, note 48). 
to take into account two insights arrived at in the following years, namely that electromagnetic fields can carry momentum (Abraham, 1902a, 1903) and that energy has mass (Einstein, 1905b). When a moving capacitor is charged, energy is transferred from the battery to the capacitor. According to special relativity, this is accompanied by a transfer of mass and momentum. The capacitor's momentum changes because its mass changes; its velocity stays the same. So the capacitor does not experience a mechanical jolt upon being charged. ${ }^{32}$

Most of Larmor's editorial note on the Trouton experiment is devoted to a new experiment that Trouton had started to work on, possibly at Larmor's own instigation. In 1903, Trouton moved to London, where he performed the experiment in collaboration with Noble, one of his students. This Trouton-Noble experiment involves a charged and carefully insulated plate capacitor hanging from the ceiling on a torsion wire. The following qualitative argumentsanctioned by the general formula (21) for the energy of an electromagnetic field - convinced Trouton that the electromagnetic energy stored in a moving charged capacitor will depend on the orientation of its plates with respect to its velocity. The charges on the moving plates correspond to currents generating magnetic fields. If the plates happen to be parallel to the earth's motion through the ether, the magnetic field of the charges on the top plate and the magnetic field of the charges on the bottom plate reinforce one another in the space between the plates and nearly cancel one another almost everywhere else. For this orientation of the plates, there will thus be a magnetic field, confined (to a good approximation) to the space between the plates. If the plates happen to be perpendicular to the earth's motion, the currents corresponding to the charged plates cancel one another and there will be no magnetic field.

Suppose the plates are connected to a battery while they are perpendicular to the ether wind. This only produces an electric field. Then the battery is disconnected, precautions are taken to prevent charge from leaking off the plates, and the capacitor is rotated over $90^{\circ}$ to put its plates parallel to the ether wind. This produces an additional magnetic field. Like FitzGerald before him, Trouton asked where the energy for this magnetic field would come from. His answer was that it could only come from mechanical energy put into the system during the rotation. When the plates go from perpendicular to parallel to the ether drift, he argued, work must be done against a torque coming from the electromagnetic forces acting on them. When unopposed, Trouton reasoned, this torque would try to put the plates perpendicular to their direction of motion, the orientation for which the electromagnetic energy is at a minimum. The capacitor in the Trouton-Noble experiment should thus act as a weather vane for ether wind. The effect would only be of order $v^{2} / c^{2}$,

${ }^{32}$ For a more detailed discussion of the Trouton experiment and its relation to $E=m c^{2}$, see (Janssen, 2003). 
but Trouton and Noble achieved remarkable accuracy. The famous MichelsonMorley experiment of 1887 put an upper limit of about $5 \mathrm{~km} / \mathrm{s}$ on the earth's velocity through the ether (the earth's velocity in its orbit around the sun is about $30 \mathrm{~km} / \mathrm{s}$ ). Trouton and Noble claimed that they would have detected any ether drift greater than $1.5 \mathrm{~km} / \mathrm{s}$. Of course, as in the Trouton experiment, they did not find any (Trouton and Noble, 1903).
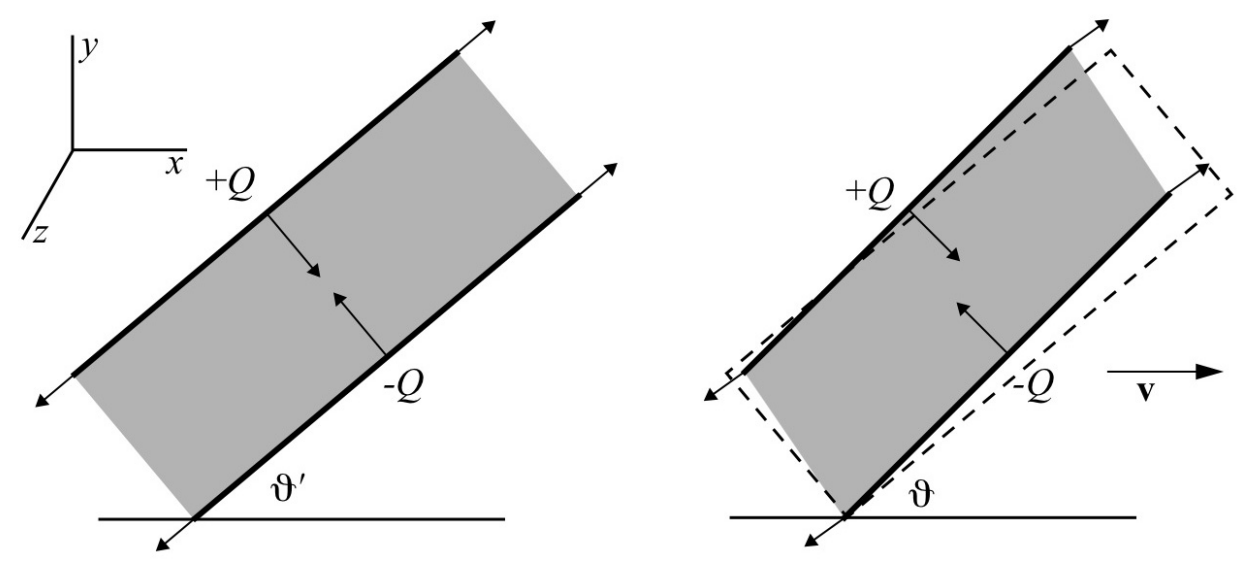

Fig. 3. Capacitor at rest (left) and same capacitor in motion having undergone the Lorentz-FitzGerald contraction (right)

The diagrams in Fig. 3 show the electromagnetic forces on a charged capacitor, the one on the left for a capacitor at rest in the ether with its plates at an angle $\vartheta^{\prime}$ with respect to the $x$-axis, the one on the right for the same capacitor with its plates at that same angle after it has been set in motion with a velocity $v$ in the $x$-direction. The moving capacitor is subject to the Lorentz-FitzGerald contraction, so the angle $\vartheta$ between its plates and its velocity is slightly greater than $\vartheta^{\prime}$. First consider the capacitor at rest in the ether. The diagram shows three pairs of Coulomb forces. First, there are the attractive forces between the opposite charges on the top and the bottom plate. These forces can be represented by two resultant forces at the centers of the plates. Then there are the repulsive forces between the positive charges on the top plate and the repulsive forces between the negative charges on the bottom plate. These forces can be represented by pairs of forces at the edges of the plates (Laue, 1912b, p. 175; Pauli, 1921, p. 129). Fig. 3 shows two such pairs in the $x y$-plane. There will be similar forces in the $z$-direction but those need not be taken into account. As long as the capacitor is at rest in the ether, none of these forces give rise to a torque.

The electromagnetic forces $\mathbf{F}$ on the contracted capacitor moving with $\mathbf{v}=$ $(v, 0,0)$ through the ether are related to the Coulomb forces $\mathbf{F}^{\prime}$ on the uncontracted capacitor at rest via (cf. Eq. (16) for $l=1$ and note 26)

$$
\mathbf{F}=\operatorname{diag}(1,1 / \gamma, 1 / \gamma) \mathbf{F}^{\prime}
$$


As Lorentz (1899, p. 432) had already noticed, this transformation law is such that forces perpendicular to a given surface in a system at rest will be perpendicular to the corresponding surface in the contracted system in motion. The three pairs of forces shown on the right of Fig. 3 thus exert torques all trying to align the plates with the direction of motion. Since the Lorentz-FitzGerald contraction is a second order effect, these torques will only be of order $v^{2} / c^{2}$. The direction of these torques is the opposite of what Trouton expected on the basis of his energy considerations. They try to maximize the system's electromagnetic energy! This immediately tells us that the electromagnetic energy cannot be the only energy in the moving capacitor that depends on the orientation of the plates. More specifically, it tells us that the energy associated with the stabilizing forces that prevent the charges from flying off the plates and the plates from collapsing onto one another must similarly depend on the orientation of the plates.

This observation is key to Lorentz's explanation of the negative result of the Trouton-Noble experiment. ${ }^{33}$ For the charged capacitor at rest in the ether to be a stable system, the Coulomb repulsions and attractions shown in the diagram on the left in Fig. 3 must be fully compensated. On the assumption that all forces transform like Coulomb forces, the moving capacitor will then also be a stable a system and these counteracting forces will exert torques equal and opposite to the ones coming from the electromagnetic forces. There will be no net torque on the system. This explanation readily carries over to special relativity.

In special relativity, these delicately balanced torques are ultimately artifacts of the standard non-Lorentz-invariant definition (41) of the four-momentum of spatially extended systems in Minkowski space-time, just as the factor $4 / 3$ in the mass-energy relation (29) for Lorentz's purely electromagnetic electron. In the absence of relativistic continuum mechanics, the theoretical analysis of the Trouton-Noble experiment was particularly challenging. Two of the leading theorists of the period, Larmor and Lorentz, found conflicting results. It is a non-trivial task to figure out what lies behind these discrepancies. Sec. 4.2 will give the analysis in terms of energy; sec. 4.3 in terms of momentum. Under the standard definitions of energy and momentum, there will be equal and opposite torques coming from the electromagnetic field and the material part of the moving capacitor, as well as exchanges of energy and momentum between these two parts whenever the system is rotated. Under the alternative Lorentz-invariant definitions (see Eq. (42)) there are no such torques and there is no such flow of energy or momentum. As I shall argue in more detail in sec. 4.4 , this is a clear indication that these are all kinematical effects.

$\overline{33}$ This explanation is implicit in (Lorentz, 1904b, p. 29) and is made explicit in (Laue, 1911a) (Janssen, 1995, sec. 1.4). 


\subsection{Torques or no torques on a moving capacitor when calculated in terms of energy}

Using Lorentz's theorem of corresponding states, Larmor (1902) argued that there is a simple relation between the electromagnetic energy $U_{\mathrm{EM}}$ in a capacitor moving through the ether at velocity $\mathbf{v}$ and the electric energy $U_{\mathrm{EM}}^{\prime}$ in a corresponding capacitor at rest in the ether carrying the same charges as the moving capacitor but stretched by a factor $\gamma$ in the direction of motion. This relation,

$$
U_{\mathrm{EM}}=U_{\mathrm{EM}}^{\prime} / \gamma,
$$

forms the basis for a geometrical argument showing that the electromagnetic forces on a moving capacitor produce a torque if the system is not subject to the Lorentz-FitzGerald contraction, but that there is no torque if it does contract. Larmor thus saw the Trouton-Noble experiment as a test of the contraction hypothesis and its negative result as a confirmation of the hypothesis.

Consider the two-dimensional representation of the situation in Fig. 3. With the Lorentz-FitzGerald contraction, the moving capacitor is a parallelogram the shape of which depends on $\vartheta$, while the corresponding capacitor at rest is always a rectangle of the same shape - at different angles $\vartheta^{\prime}$, to be sure, but orientation makes no difference at rest in the ether. So $U_{\mathrm{EM}}^{\prime}$ is independent of $\vartheta^{\prime}$. This implies, through Eq. (51), that $U_{\mathrm{EM}}$ is independent of $\vartheta$. The energy of a contracted moving capacitor will not depend on the angle between its plates and its velocity. Such a capacitor can be rotated without energy entering or leaving the system. With the Lorentz-FitzGerald contraction, in other words, there is no torque.

The situation is different if systems set in motion through the ether were to retain their shape. Without the Lorentz-FitzGerald contraction, the moving capacitor is a rectangle of the same shape for all values of $\vartheta$, while the corresponding capacitor at rest is a parallelogram the shape of which changes with $\vartheta$. These capacitors of different shapes at rest will have different energies $U_{\mathrm{EM}}^{\prime}$. According to Eq. (51), the energy $U_{\mathrm{EM}}$ of an uncontracted moving capacitor will then depend on the orientation of its plates. Without the Lorentz-FitzGerald contraction, in short, there should be a torque.

What is the direction of this torque? When the plates are perpendicular to the velocity $\left(\vartheta=90^{\circ}\right)$, stretching the uncontracted moving capacitor increases the distance between the plates, putting the opposite charges on them further apart. In this case, the stretching increases the potential energy. When the plates are parallel to the velocity $\left(\vartheta=0^{\circ}\right)$, the stretching does not affect the distance between the plates but increases the size of both plates, putting the like charges on them further apart. In this case, the stretching decreases the potential energy. According to Larmor, the orientation without a magnetic 
field $\left(\vartheta=90^{\circ}\right)$ would thus be more energetic than the orientation with a magnetic field $\left(\vartheta=0^{\circ}\right)$. Trouton, understandably, expected just the opposite. In fact, the experimentalists did not accept the energy considerations of their theoretical advisor and stuck to their guns in (Trouton and Noble, 1903, p. 165 , note). At least, Larmor's analysis leads to a torque in the same direction as the analysis directly in terms of forces (see Fig. 3). According to Larmor, however, there will only be a torque if there is no Lorentz-FitzGerald contraction. The analysis in terms of forces leads to the same torque with and without contraction. ${ }^{34}$

The standard definition (21) of the energy of an electromagnetic field gives a different result for the energy of the field of a moving contracted capacitor than Larmor's Eq. (51): ${ }^{35}$

$$
U_{\mathrm{EM}}=U_{\mathrm{EM}}^{\prime} / \gamma+2 U_{\mathrm{EM}}^{\prime} \gamma \beta^{2} \cos ^{2} \vartheta^{\prime} .
$$

As mentioned above, this expression sanctions Trouton's conclusion that the electromagnetic energy in a moving capacitor has a minimum when the plates are perpendicular to their velocity $\left(\vartheta^{\prime}=\vartheta=90^{\circ}\right)$.

Both Eq. (51) and Eq. (52) look odd from a relativistic point of view. If the energy were to transform as the first component of a four-vector (see Eq. (38)), the relation between $U_{\mathrm{EM}}$ and $U_{\mathrm{EM}}^{\prime}$ for a Lorentz boost setting the system in motion in the $x$-direction with velocity $v$ would be:

$$
U_{\mathrm{EM}}=\gamma U_{\mathrm{EM}}^{\prime} .
$$

Where do the discrepancies between Eqs. (51), (52), and (53) come from? To answer this question, it will be helpful to reconstruct Larmor's derivation of Eq. (51). ${ }^{36}$ Larmor set the energy $U_{\mathrm{EM}}$ equal to the work needed to charge the moving capacitor. This is the integral from $q=0$ to $q=Q$ of the work $d W(q)$ needed to add the infinitesimal charge $d q$ to the plates when there already is

\footnotetext{
${ }^{34}$ In the stretched capacitor at rest corresponding to an uncontracted moving capacitor, the resultant forces at the centers and at the edges of the plates will not always be perpendicular to the plates and parallel to the plates, respectively (Janssen, 1995, sec. 1.2.2).

${ }^{35}$ The electric field in the capacitor at rest is perpendicular to the plates (cf. Fig. $3): \mathbf{E}^{\prime}=E^{\prime}\left(\sin \vartheta^{\prime},-\cos \vartheta^{\prime}, 0\right)$. There is no magnetic field: $\mathbf{B}^{\prime}=0$. The energy of this electromagnetic field is: $U_{\mathrm{EM}}^{\prime}=\int \frac{1}{2} \varepsilon_{0} E^{\prime 2} d^{3} x^{\prime}$. The field in the moving capacitor is the Lorentz transform of this field (see Eq. (15) for $l=1)$ : $\mathbf{E}=E^{\prime}\left(\sin \vartheta^{\prime},-\gamma \cos \vartheta^{\prime}, 0\right)$ and $\mathbf{B}=E^{\prime}\left(0,0,-\gamma\left(v / c^{2}\right) \cos \vartheta^{\prime}\right)$. Inserting these expressions into Eq. (21) and using that the volume element $d^{3} x$ can be replaced by $d^{3} x^{\prime} / \gamma$ (cf. the discussion following Eq. (47)), one arrives at Eq. (52).

${ }^{36}$ For discussion of the relevant rather cryptic passage in (Larmor, 1902) and a more detailed version of the derivation, see (Janssen, 1995, sec. 1.3; sec. 1.4; and sec. 2.4, note 75$)$.
} 
a charge $q$ on them $(+q$ on one, $-q$ on the other $)$ :

$$
U_{\mathrm{EM}}=\int_{0}^{Q} d W(q)
$$

The integrand can be written as

$$
d W(q)=\int_{\text {bottom }}^{\text {top }} \mathbf{F}(q) \cdot d \mathbf{s}
$$

where $\mathbf{F}(q)$ is the force exerted on $d q$ by the electromagnetic field produced by the charge $q$ already on the plates as $d q$ is transferred from one plate to the other along some arbitrary path $\mathbf{s}$ (with infinitesimal segments $d \mathbf{s}$ ). Larmor related the work $d W(q)$ done in the moving capacitor to the work $d W^{\prime}(q)$ done in a stretched capacitor at rest in the ether when $d q$ is transferred along the corresponding path $\mathbf{s}^{\prime}$. The segments of these two paths will be related to one another via $d \mathbf{s}=\operatorname{diag}(1 / \gamma, 1,1) d \mathbf{s}^{\prime}$. Using Eq. (50) to relate the forces in these two situations, one finds a simple relation between $d W(q)$ and $d W^{\prime}(q)$ :

$$
d W(q)=\int_{\text {bottom }}^{\text {top }} \operatorname{diag}(1,1 / \gamma, 1 / \gamma) \mathbf{F}^{\prime}(q) \cdot \operatorname{diag}(1 / \gamma, 1,1) d \mathbf{s}^{\prime}=d W^{\prime}(q) / \gamma .
$$

Inserting this result into Eq. (54), one recovers Larmor's Eq. (51):

$$
U_{\mathrm{EM}}=\int_{0}^{Q} d W^{\prime}(q) / \gamma=U_{\mathrm{EM}}^{\prime} / \gamma
$$

From a relativistic point of view, Larmor failed to take into account that the energy of the electromagnetic field of a moving capacitor has mass and momentum, just as he failed to take this into account in his analysis of the Trouton experiment. To put it differently: he neglected the kinetic energy of the extra mass in motion. This kinetic energy is equal to the work $W_{P_{\mathrm{EM}}}$ needed to build up the electromagnetic momentum of the moving capacitor:

$$
W_{P_{\mathrm{EM}}}=\int_{0}^{Q} \frac{d \mathbf{P}_{\mathrm{EM}}(q)}{d t} \cdot d \mathbf{s}=\int_{0}^{Q} \frac{d}{d q}\left(\mathbf{P}_{\mathrm{EM}}(q) \cdot \frac{d \mathbf{s}}{d t}\right) d q=\mathbf{P}_{\mathrm{EM}}(Q) \cdot \mathbf{v} .
$$

This energy needs to be added to the right-hand side of Eq. (57). If the energy and momentum of the electromagnetic field of the capacitor are defined in such a way that they transform as the components of a four-vector under Lorentz transformations, the momentum of the electromagnetic field of a moving charged capacitor is given by:

$$
\mathbf{P}_{\mathrm{EM}}=\gamma\left(U_{\mathrm{EM}}^{\prime} / c^{2}\right) \mathbf{v}
$$

In that case,

$$
W_{P_{\mathrm{EM}}}=\gamma \beta^{2} U_{\mathrm{EM}}^{\prime}
$$

which, when added to the right-hand side of Eq. (57), leads to the relativistic 
relation (53):

$$
U_{\mathrm{EM}}=U_{\mathrm{EM}}^{\prime} / \gamma+\gamma \beta^{2} U_{\mathrm{EM}}^{\prime}=\gamma U_{\mathrm{EM}}^{\prime}
$$

This new expression is still independent of $\vartheta$, so the correction to Larmor's formula (51) does not affect the argument on the basis of which he concluded that the electromagnetic forces on a moving charged capacitor do not give a torque as long as the capacitor is subject to the Lorentz-FitzGerald contraction.

If $\left(U_{\mathrm{EM}} / c, \mathbf{P}_{\mathrm{EM}}\right)$ form a four-vector, one can actually pinpoint and correct the error in Larmor's Eq. (51) making use only of the transformation properties of four-vectors. Once again, consider the Lorentz boost setting the system moving with velocity $v$ in the $x$-direction (cf. Eqs. (14) and (37)). As Lorentz routinely did with his theorem of corresponding states (cf. sec. 2.2), one can do this in two steps, first going to a system of Galilean-transformed quantities $\left(c t^{\prime}, \mathbf{x}^{\prime} \rightarrow c \hat{t}, \hat{\mathbf{x}}\right)$ and then to the system of Lorentz-transformed quantities $(c \hat{t}, \hat{\mathbf{x}} \rightarrow c t, \mathbf{x})$. The non-trivial parts of these transformations are given by

$$
c \hat{t}=c t^{\prime}, \quad \hat{x}=x^{\prime}+\beta c t^{\prime},
$$

and

$$
c t=\frac{c \hat{t}}{\gamma}+\gamma \beta \hat{x}, \quad x=\gamma \hat{x},
$$

respectively. Composition of the two reproduces the familiar equations for the Lorentz boost $\left(c t^{\prime}, \mathbf{x}^{\prime} \rightarrow c t, \mathbf{x}\right)$ (Eq. (14) for $\left.l=1\right)$. If energy and momentum $\left(U_{\mathrm{EM}} / c, \mathbf{P}_{\mathrm{EM}}\right)$ transform the same way as the time and space coordinates $(c t, \mathbf{x})$, one has, in analogy with the first relation in Eq. (63):

$$
\frac{U_{\mathrm{EM}}}{c}=\frac{1}{\gamma} \frac{\hat{U}_{\mathrm{EM}}}{c}+\gamma \beta \hat{P}_{\mathrm{EM}_{x}} .
$$

Larmor's Eq. (51) is obtained when $\hat{P}_{\mathrm{EM}_{x}}$ is set equal to zero. Pre-relativistically, before it was realized that moving energy has momentum, this was a natural thing to do. In analogy with the second relation in Eq. (62), however, one has

$$
\hat{P}_{\mathrm{EM}_{x}}=P_{\mathrm{EM}_{x}}^{\prime}+\beta \frac{U_{\mathrm{EM}}^{\prime}}{c}=\beta \frac{U_{\mathrm{EM}}^{\prime}}{c} .
$$

Since the primed system is at rest in the ether, $P_{\mathrm{EM}_{x}}^{\prime}=0$, which was used in the second step in Eq. (65). Note that the momentum $\hat{\mathbf{P}}_{\mathrm{EM}}$ in the 'Galileanboosted' system is just the product of the rest mass $\left(U_{\mathrm{EM}}^{\prime} / c^{2}\right)$ and the velocity v. Substituting $\beta\left(U_{\mathrm{EM}}^{\prime} / c\right)$ for $\hat{P}_{\mathrm{EM}_{x}}$ into Eq. (64) along with $\hat{U}_{\mathrm{EM}}=U_{\mathrm{EM}}^{\prime}$, the analogue of the first relation in Eq. (62), one recovers the standard relativistic Eq. (53) (cf. Eqs. (60)-(61)).

This standard relation, however, only obtains if the energy and momentum of the electromagnetic field of the capacitor transform as a four-vector. Under 
the standard definitions (21)-(22) they do not. For the electromagnetic field of a moving charged capacitor (see note 35), Eq. (22) gives:

$$
\mathbf{P}_{\mathrm{EM}}=2\left(U_{\mathrm{EM}}^{\prime} / c\right) \beta \cos \vartheta^{\prime}\left(\gamma \cos \vartheta^{\prime}, \sin \vartheta^{\prime}, 0\right)
$$

While the momentum $\mathbf{P}_{\mathrm{EM}}$ in Eq. (59) is in the direction of motion, regardless of the orientation of the plates of the capacitor with respect to its velocity, the momentum $\mathbf{P}_{\mathrm{EM}}$ in Eq. (66) is roughly in the direction of the plates (to order $v / c, \gamma=1$ and $\vartheta^{\prime}=\vartheta$ ). According to Eq. (58), the amount of work needed to build up the momentum in Eq. (66) is:

$$
W_{P_{\mathrm{EM}}}=2 U_{\mathrm{EM}}^{\prime} \gamma \beta^{2} \cos ^{2} \vartheta^{\prime}
$$

Adding this to the right-hand side of Larmor's Eq. (51), one recovers Eq. (52) based on the standard definition of the energy of an electromagnetic field.

Eq. (52) can be rewritten as:

$$
U_{\mathrm{EM}}=U_{\mathrm{EM}}^{\prime}\left(\gamma-\gamma \beta^{2} \sin ^{2} \vartheta^{\prime}+\gamma \beta^{2} \cos ^{2} \vartheta^{\prime}\right)
$$

Following the general treatment of moving stressed bodies in (Einstein, 1907b, pp. 373-377), one can connect the $\vartheta^{\prime}$-dependent terms that distinguish this expression from expression (53) directly to the relativity of simultaneity (Janssen, 1995, sec. 2.4). Consider Fig. 4, which has two pairs of electromagnetic forces

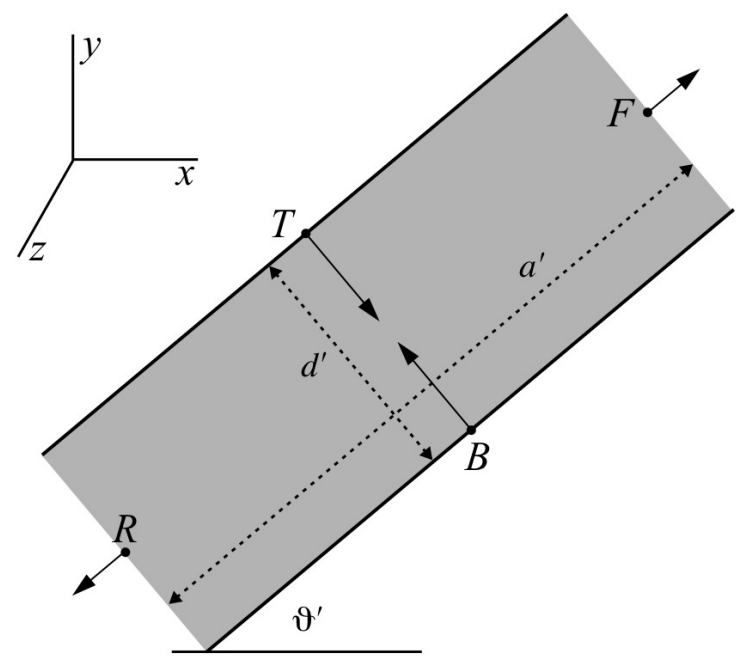

Fig. 4. Electromagnetic forces on a capacitor in its rest frame.

on a capacitor in its rest frame, consolidating the two pairs in Fig. 3 at the edges of both plates into one pair in between. Assume that the plates of the capacitor in its rest frame are squares with sides $a^{\prime}$ that are much larger than the distance $d^{\prime}$ between the plates. The larger the ratio $a^{\prime} / d^{\prime}$, the better the approximation that the field is constant and confined to the space between the plates. In that approximation the size of the resultant forces at the centers 
of the plates is $U_{\mathrm{EM}}^{\prime} / d^{\prime}$ and the size of the forces at the sides of the capacitor is $U_{\mathrm{EM}}^{\prime} / a^{\prime}$. The forces at the centers are perpendicular to the plates, those at the sides are parallel to the plates. So the forces at the rear $(R)$ and the top $(T)$ are given by: ${ }^{37}$

$$
\mathbf{F}^{\prime R}=\frac{U_{\mathrm{EM}}^{\prime}}{a^{\prime}}\left(-\cos \vartheta^{\prime},-\sin \vartheta^{\prime}, 0\right), \quad \mathbf{F}^{\prime T}=\frac{U_{\mathrm{EM}}^{\prime}}{d^{\prime}}\left(\sin \vartheta^{\prime},-\cos \vartheta^{\prime}, 0\right) .
$$

The forces at the front $(F)$ and the bottom $(B)$ are the opposite of those at $R$ and $T$, respectively. On the assumption that the capacitor is charged in its rest frame, these four forces are switched on simultaneously in that frame. This means, as Einstein (1907b) pointed out, that they are not switched on simultaneously in other frames, such as the one in which the capacitor is moving at velocity $v$ in the $x$-direction. In this frame there will be a delay of $\Delta t_{R F}=\gamma\left(v / c^{2}\right) \Delta x_{R F}^{\prime}$ between the moment $\mathbf{F}^{R}$ is switched on and the moment $\mathbf{F}^{F}$ is switched on, where $\Delta x_{R F}^{\prime}=a^{\prime} \cos \vartheta^{\prime}$ is the distance between $R$ and $F$ in the rest frame (cf. Eq. (4)). There will likewise be a delay of $\Delta t_{T B}$ between the forces at $T$ and $B$ being switched on:

$$
\Delta t_{R F}=\gamma\left(v / c^{2}\right) a^{\prime} \cos \vartheta^{\prime}, \quad \Delta t_{T B}=\gamma\left(v / c^{2}\right) d^{\prime} \sin \vartheta^{\prime}
$$

During the short period of time that the forces at $R$ and $T$ are not compensated by those at $F$ and $B$, an amount of work

$$
\Delta W=F_{x}^{R} v \Delta t_{R F}+F_{x}^{T} v \Delta t_{T B}
$$

is done at the expense of the energy of the system. According to the transformation law (50) for forces, $F_{x}=F_{x}^{\prime}$. Inserting Eqs. (69) and (70) into Eq. (71), one finds that the energy $\Delta U_{\mathrm{EM}}^{\prime}=-\Delta W$ associated with this effect is exactly equal to the $\vartheta^{\prime}$-dependent terms in Eq. (68). The forces compensating the electromagnetic forces are subject to this same effect and contribute an amount of energy equal and opposite to $\Delta U_{\mathrm{EM}}^{\prime}$. The total energy of the system will thus be independent of $\vartheta^{\prime}$.

These considerations provide an exhaustive answer to the question where the discrepancies between Eqs. (51), (52), and (53) for $U_{\mathrm{EM}}$ come from. To recap: the problem with Larmor's Eq. (51) is that it fails to take into account the energy needed to build up the electromagnetic momentum in the moving capacitor. This problem can be solved either with the help of the standard definition (41) for the momentum of spatially extended systems or with the help of the alternative covariant definition (42). Adopting the standard definition, one finds Eq. (52). Adopting the alternative definition, one finds Eq. (53). The

$\overline{37}$ These values result if the expression (78) below for the Maxwell stress tensor for the electromagnetic field of the capacitor in its rest frame is inserted into the general expression $F^{\prime i}=-\int \partial_{j}^{\prime} T^{\prime i j} d^{3} x^{\prime}$ for the components of the force on a static system in its rest frame (cf. note 26 ). 
difference between Eqs. (52) and (53) can be interpreted directly in terms of the relativity of simultaneity (see Eqs. (68)-(71)), as one would expect since the only difference between the standard definition and the alternative definition is that one involves hyperplanes of simultaneity in the observer's rest frame while the other involves hyperplanes of simultaneity in the capacitor's rest frame.

\subsection{Torques or no torques on a moving capacitor when calculated in terms of momentum}

Lorentz (1904b) analyzed both the Trouton and the Trouton-Noble experiment in terms of electromagnetic momentum. In both cases, his conclusions differed from Larmor's. I focus on the Trouton-Noble experiment. ${ }^{38}$ According to Lorentz, the electromagnetic forces on a charged moving capacitor form a torque, whether or not the moving capacitor contracts. However, only if it does contract, Lorentz argued, will the torque coming from the electromagnetic forces be compensated by a torque coming from the stabilizing forces that keep the charges on the plates and prevent the plates from collapsing onto one another. This compensating torque arises because the contraction hypothesis requires that these stabilizing forces transform as Coulomb forces under Lorentz transformations. So Lorentz and Larmor agreed to the extent that the explanation of the Trouton-Noble experiment calls for the Lorentz-FitzGerald contraction hypothesis. They disagreed about the role of the hypothesis in the explanation.

Lorentz's derivation for the formula of the torque of the electromagnetic forces on a moving capacitor makes use of results presented in detail in (Lorentz, 1904a). In analogy with the momentum of the electromagnetic field of a capacitor, one can define its angular momentum:

$$
\mathbf{L}_{\mathrm{EM}}=\int \mathbf{x} \times \mathbf{p}_{\mathrm{EM}} d^{3} x .
$$

The integral is evaluated in a frame at rest in the ether. The torque exerted on the capacitor is minus the time derivative of this quantity:

$$
\mathbf{T}_{\mathrm{EM}}=-\frac{d}{d t} \int \mathbf{x} \times \mathbf{p}_{\mathrm{EM}} d^{3} x
$$

For a system such as a capacitor and its electromagnetic field that is static except for an overall constant velocity $\mathbf{v}$, this reduces to:

$$
\mathbf{T}_{\mathrm{EM}}=\mathbf{P}_{\mathrm{EM}} \times \mathbf{v},
$$

\footnotetext{
${ }^{38}$ See (Janssen, 2002a, appendix; 2003) for discussion of the case of the Troutonexperiment.
} 
where $\mathbf{P}_{\mathrm{EM}}$ is the electromagnetic momentum in the standard definition (22). As can be seen from Eq. (66), this momentum is roughly in the direction of the plates of the capacitor. The torque (74) tries to pull the plates into the direction of motion. In special relativity, Eq. (74) holds for the spatial part of the four-momentum of any system that is static but for an overall constant velocity $\mathbf{v}$. In particular, it holds for the momentum $\mathbf{P}_{\text {stab }}$ of the stabilizing mechanism, the analogue of the Poincaré stresses, which in this case is provided by the material part of the capacitor rather than by some mysterious ether suction. It can even be shown (Janssen, 1995, sec. 2.2.5) that Eq. (74) continues to hold for all such systems if the standard definition (41) of the four-momentum of spatially extended systems is replaced by definition $(42)$.

The two definitions coincide for closed systems, such as the capacitor and its electromagnetic field. Since the total four-momentum, $P_{\text {tot }}^{\mu}$, transforms as a four-vector under Lorentz transformations, its spatial part, $\mathbf{P}_{\text {tot }}$, is in the direction of motion and there will be no net torque:

$$
\mathbf{T}_{\text {tot }}=\mathbf{P}_{\text {tot }} \times \mathbf{v}=0
$$

Under the covariant definition (42), the momenta $\mathbf{P}_{\mathrm{EM}}^{\mathrm{cov}}$ and $\mathbf{P}_{\mathrm{stab}}^{\mathrm{cov}}$ of the open subsystems are also in the direction of motion and there will be no torques whatsoever:

$$
\mathbf{T}_{\mathrm{tot}}^{\mathrm{cov}}=\mathbf{T}_{\mathrm{EM}}^{\mathrm{cov}}=\mathbf{T}_{\mathrm{stab}}^{\mathrm{cov}}=0
$$

Under the standard definition, neither $\mathbf{P}_{\mathrm{EM}}$ nor $\mathbf{P}_{\text {stab }}$ are in the direction of motion and there will be two equal and opposite torques:

$$
\mathbf{T}_{\text {tot }}^{\text {stan }}=0, \quad \mathbf{T}_{\mathrm{EM}}^{\mathrm{stan}}=-\mathbf{T}_{\mathrm{stab}}^{\mathrm{stan}} \neq 0 \text {. }
$$

The standard relativistic treatment of the Trouton-Noble experiment, which is due to Laue $(1911 \mathrm{a}, \mathrm{b})$ and which is the one presented by Pauli (1921, sec. 44) and most subsequent authors, is based on the standard definition of fourmomentum and thus involves these delicately balanced torques. On the face of it, this relativistic explanation is not much different from Lorentz's ethertheoretic explanation.

Unlike Lorentz, however, Laue clearly recognized that these opposing torques are a generic feature of closed systems in motion that can somehow be divided into open subsystems. ${ }^{39}$ Laue (1912a) pointed out, for instance, that

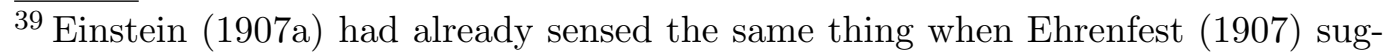
gested that a moving electron would experience a torque if an electron at rest were an ellipsoid rather than a sphere. Einstein's position was that the electron would behave exactly the same regardless of its shape. This position was vindicated by Laue's work a few years later. For discussion of the exchange between Einstein and
} 
the physics of a thought experiment proposed by Lewis and Tolman (1909) involving a torque on a moving bent lever is essentially the same as the physics of the Trouton-Noble experiment. ${ }^{40}$ It was only after Rohrlich's work in the 1960s, that it was recognized that one can eliminate the delicately balanced torques in Laue's accounts of the Trouton-Noble capacitor and the LewisTolman bent lever altogether by adopting the alternative definition (42) of the four-momentum of spatially extended systems in Minkowski space-time (Butler, 1968; Janssen, 1995; Teukolsky, 1996). There has been some debate in the literature on the Trouton-Noble experiment - as there has been in the far more extensive literature on the classical electron model (see sec. 3.4) over what the correct definition is. ${ }^{41}$ Since the difference between the two is merely a convention about the choice of spatial hyperplanes in the general definition (40), this cannot be a matter of right or wrong but only of whether one definition is perhaps more convenient, more elegant, or more natural than the other. The analysis in sec. 4.2 of different expressions for $U_{\mathrm{EM}}$ makes it doubtful that one can even claim that much on behalf of one or the other. After all, expressions (52) and (53) for $U_{\mathrm{EM}}$, based on definitions (41) and (42) of $P^{\mu}$, respectively, could both be given perfectly sensible physical interpretations in terms of the work done in charging a moving capacitor.

It will be instructive to derive the expressions for the four-momenta of the electromagnetic field and the stabilizing mechanism under the standard definition (41). As in the case of the Lorentz-Poincaré electron (see sec. 3.4), the starting point will be the energy-momentum tensor for the electromagnetic field in the system at rest. The electron at rest is spherically-symmetric. The capacitor is not, which complicates matters. It will be convenient to start with a capacitor at rest with its plates parallel to the $x z$-plane $\left(\vartheta^{\prime}=0\right.$ in Fig. 3$)$. As before (cf. Fig. 4), it will be assumed that the area of the plates $\left(l^{2}\right.$ if they are squares with sides $l^{\prime}$ ) is very large compared to the distance $d^{\prime}$ between them $\left(l^{\prime} \gg d^{\prime}\right)$. If the top plate carries the positive charge, there will be an electric field pointing in the negative $y$-direction, which, to a very good approximation, will be homogeneous and confined to the space between the plates: $\mathbf{E}^{\prime}=\left(0,-E^{\prime}, 0\right)$. The energy density will similarly have the constant value $u_{\mathrm{EM}}^{\prime}=\frac{1}{2} \varepsilon_{0} E^{\prime 2}$ inside the capacitor and vanish outside. The Maxwell stress tensor (44) for this field is given by:

$$
T_{\text {Max }}^{\prime i j}=\operatorname{diag}(-1,1,-1) u_{\mathrm{EM}}^{\prime} \Theta\left(x^{\prime}, y^{\prime}, z^{\prime}\right),
$$

where $\Theta\left(x^{\prime}, y^{\prime}, z^{\prime}\right)$ is shorthand for some combination of step-functions such that $\Theta=1$ inside and $\Theta=0$ outside the capacitor. The energy-momentum

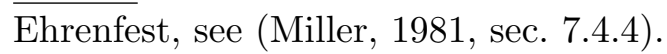

40 This connection with the Lewis-Tolman bent lever is mentioned in (Pauli, 1921, p. 128). For discussion of the example of the bent lever, see, e.g., (Norton, 1992, sec. 9) or (Janssen, 1995, sec. 2.5.1).

${ }^{41}$ See, especially, the rebuttal by Singal (1993) of (Butler, 1968). 
tensor for this field is (cf. Eq. (43)):

$$
T_{\mathrm{EM}}^{\prime \mu \nu}=\left(\begin{array}{cc}
u_{\mathrm{EM}}^{\prime} & 0 \\
0 & -T_{\mathrm{Max}}^{\prime i j}
\end{array}\right)=\operatorname{diag}(1,1,-1,1) u_{\mathrm{EM}}^{\prime} \Theta\left(x^{\prime}, y^{\prime}, z^{\prime}\right) .
$$

The corresponding four-momentum is

$$
P_{\mathrm{EM}}^{\prime \mu}=\frac{1}{c} \int T_{\mathrm{EM}}^{\prime \mu 0} d^{3} x^{\prime}=\left(U_{\mathrm{EM}}^{\prime} / c, 0,0,0\right)
$$

If the appropriate energy-momentum tensor for the stabilizing mechanism is added to the energy-momentum tensor for the field, the four-divergence of their sum will vanish, indicating that the combined system is closed:

$$
\partial_{\nu}^{\prime}\left(T_{\mathrm{EM}}^{\prime \mu \nu}+T_{\mathrm{stab}}^{\prime \mu \nu}\right)=0
$$

This equation (and the assumption that $T_{\text {stab }}^{\prime \mu \nu} \rightarrow 0$ for $|\mathbf{x}| \rightarrow \infty$ ) uniquely determines the $i j$-components of $T_{\text {stab }}^{\prime \mu \nu}$ whatever the exact nature of the stabilizing mechanism may turn out to be. Since the system is static, the $i 0$ and $0 i$-components must be zero. The 00-component can be any function of $\left(x^{\prime}, y^{\prime}, z^{\prime}\right)$ as long as the total energy density $T_{\text {tot }}^{\prime 00}$ remains positive definite. It will be convenient to set this component to zero as well. ${ }^{42}$ One thus arrives at:

$$
T_{\mathrm{stab}}^{\prime \mu \nu}=\operatorname{diag}(0,-1,1,-1) u_{\mathrm{EM}}^{\prime} \Theta\left(x^{\prime}, y^{\prime}, z^{\prime}\right)
$$

Since $T_{\text {stab }}^{\prime \mu 0}=0$, it follows that $P_{\text {stab }}^{\prime \mu}=0$ as well.

Now rotate the plates of the capacitor around the $z$-axis over an angle $\vartheta^{\prime}$ and set the whole system in motion with velocity $v$ in the $x$-direction. The transformation matrix for this rotation-followed-by-a-boost is:

$$
\Lambda_{\nu}^{\mu}=\left(\begin{array}{cccc}
\gamma & \gamma \beta & 0 & 0 \\
\gamma \beta & \gamma & 0 & 0 \\
0 & 0 & 1 & 0 \\
0 & 0 & 0 & 1
\end{array}\right)\left(\begin{array}{cccc}
1 & 0 & 0 & 0 \\
0 & \cos \vartheta^{\prime} & -\sin \vartheta^{\prime} & 0 \\
0 & \sin \vartheta^{\prime} & \cos \vartheta^{\prime} & 0 \\
0 & 0 & 0 & 1
\end{array}\right)
$$

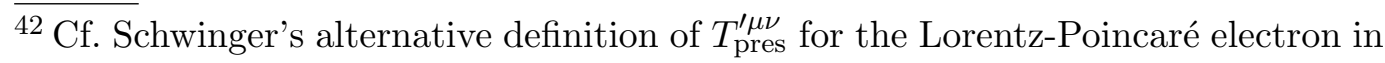
note ??. 
Proceeding as in Eqs. (47)-(48), using Eqs. (79) and (83), one finds

$$
P_{\mathrm{EM}}^{\mu}=\frac{1}{c} \int \Lambda_{\rho}^{\mu} \Lambda_{\sigma}^{0} T_{\mathrm{EM}}^{\prime \rho \sigma} d^{3} x=\frac{U_{\mathrm{EM}}^{\prime}}{c}\left(\begin{array}{c}
\gamma+\gamma \beta^{2} \cos ^{2} \vartheta^{\prime}-\gamma \beta^{2} \sin ^{2} \vartheta^{\prime} \\
2 \beta \gamma \cos ^{2} \vartheta^{\prime} \\
2 \beta \cos \vartheta^{\prime} \sin \vartheta^{\prime} \\
0
\end{array}\right)
$$

for the four-momentum of the capacitor's electromagnetic field. Eq. (84) combines Eq. (68) for the energy $U_{\mathrm{EM}}$ of the field of the capacitor and Eq. (66) for its momentum $\mathbf{P}_{\mathrm{EM}}$. Using Eqs. (82) and (83), one similarly finds

$$
P_{\mathrm{stab}}^{\mu}=\frac{1}{c} \int \Lambda_{\rho}^{\mu} \Lambda_{\sigma}^{0} T_{\mathrm{stab}}^{\prime \rho \sigma} d^{3} x=\frac{U_{\mathrm{EM}}^{\prime}}{c}\left(\begin{array}{c}
\gamma \beta^{2} \sin ^{2} \vartheta^{\prime}-\gamma \beta^{2} \cos ^{2} \vartheta^{\prime} \\
\gamma \beta \sin ^{2} \vartheta^{\prime}-\gamma \beta \cos ^{2} \vartheta^{\prime} \\
-2 \beta \cos \vartheta^{\prime} \sin \vartheta^{\prime} \\
0
\end{array}\right)
$$

for the four-momentum of the stabilizing mechanism. These four-momenta are clearly not the Lorentz transforms of $P_{\mathrm{EM}}^{\prime \mu}$ and $P_{\text {stab }}^{\prime \mu}$. For every angle $\vartheta^{\prime}$, however, their sum, $P_{\text {tot }}^{\mu}=P_{\mathrm{EM}}^{\mu}+P_{\mathrm{stab}}^{\mu}$, is the Lorentz transform of $P_{\text {tot }}^{\prime \mu}$ :

$$
P_{\mathrm{tot}}^{\mu}=\frac{1}{c} \int \Lambda_{\rho}^{\mu} \Lambda_{\sigma}^{0}\left(T_{\mathrm{EM}}^{\prime \rho \sigma}+T_{\mathrm{stab}}^{\prime \rho \sigma}\right) d^{3} x=\left(\gamma \frac{U_{\mathrm{EM}}^{\prime}}{c}, \gamma\left(\frac{U_{\mathrm{EM}}^{\prime}}{c^{2}}\right) \mathbf{v}\right) .
$$

This is not surprising given that the field and the stabilizing mechanism together form a closed system.

The only contribution to the integral in Eq. (86) comes from $T_{\mathrm{EM}}^{\prime 00}$. From Eqs. (79) and (82) one sees that $T_{\mathrm{EM}}^{\prime i j}+T_{\mathrm{stab}}^{\prime i j}=0$. A weaker result that nonetheless has the exact same effect on $P_{\text {tot }}^{\mu}$ holds for arbitrary closed static systems. For any such system, the integral over all of space of the sum of all stresses in the rest frame will vanish. ${ }^{43}$ If $T^{\mu \nu}=T_{1}^{\mu \nu}+T_{2}^{\mu \nu}$ with $\partial_{\nu} T^{\mu \nu}=0$, then

$$
\int\left(T_{1}^{\prime i j}+T_{2}^{\prime i j}\right) d^{3} x^{\prime}=0
$$

This was first shown by Laue (1911a, p. 152) and the result is sometimes referred to as 'Laue's theorem' (Miller, 1981, p. 352). It guarantees that the four-momentum of a closed static system (under the standard definition (41)) transforms as a four-vector. It also shows that deviations from $\left(\gamma U_{\mathrm{EM}}^{\prime} / c, \gamma\left(U_{\mathrm{EM}}^{\prime} / c^{2}\right) \mathbf{v}\right)$ in Eq. (84) for $P_{\mathrm{EM}}^{\mu}$ and all of $P_{\text {stab }}^{\mu}$ in Eq. (85), the

${ }^{43} \mathrm{~A}$ non-trivial example is provided by $T_{\mathrm{EM}}^{\prime i j} \neq-T_{\mathrm{pres}}^{\prime i j}$ in Eqs. (44) and (45) for the Lorentz-Poincaré electron. 
components of which are equal and opposite to those deviations, can be traced to stresses in the rest frame. Under the standard definition (41) of four-momentum, it is a general rule that stresses in the rest frame give rise to momentum in a frame in which the system is moving. Such momentum is typically not in the direction of motion. This is about as bizarre as a pin in a bowling alley falling over sideways after being hit head-on by a bowling ball. It is also what gives rise to torques (see Eq. (74)).

Laue (1911b, p. VI; 1912b) wanted to put this odd feature of relativistic mechanics, encoded in the transformation properties of four-momentum (under the standard definition), on a par with the way in which the inertia of energy is encoded in the energy-momentum tensor. As Planck (1908) had pointed out, if $T^{\mu \nu}$ is symmetric, the momentum density $T^{i 0} / c$ is equal to the energy flow $c T^{0 i}$ divided by $c^{2}$. In hindsight, it is clear that these two features do not have the same status. The peculiar relation between stresses and momentum found by Laue is an artifact of the standard definition of four-momentum. Laue's relation is on a par, not with $E=m c^{2}$, but with length contraction and time dilation. Still, Laue deserves credit for recognizing that the effect has nothing to do with the particulars of the Trouton-Noble experiment or the Lewis-Tolman bent lever but is a generic feature of open subsystems of closed static systems in Minkowski space-time.

The $\vartheta^{\prime}$-dependence of the four-momenta (84) and (85) is an element that is new compared to the analogous case of the Lorentz-Poincaré electron. Because of the spherical symmetry of the electron in its rest frame, all momentum in the moving frame is in the direction of motion, both the momentum associated with the Maxwell stresses in the rest frame and the momentum associated with the Poincaré stresses in the rest frame (see Eq. (47) and note ??). To order $v / c$ (in which case $\vartheta^{\prime}=\vartheta$ and $\gamma=1$ ), the momentum of the moving capacitor associated with the Maxwell stresses is:

$$
\mathbf{P}_{\mathrm{EM}} \approx 2 m_{\mathrm{EM}}^{\prime} v \cos \vartheta(\cos \vartheta, \sin \vartheta, 0)
$$

where $m_{\mathrm{EM}}^{\prime} \equiv U_{\mathrm{EM}}^{\prime} / c^{2}$. This deviates sharply from the simple expression $\mathbf{P}_{\mathrm{EM}} \approx m_{\mathrm{EM}}^{\prime} \mathbf{v}$ that would obtain if $P_{\mathrm{EM}}^{\mu}$ were to transform as a four-vector. In the case of of the Lorentz-Poincaré electron, the only discrepancy was the factor $4 / 3$ (cf. Eq. (47)). In the case of the capacitor, the discrepancy is much more pronounced. As shown in Fig. 5 for $\vartheta=30^{\circ}$ and $\vartheta=60^{\circ}, \mathbf{P}_{\mathrm{EM}}$ is a vector parallel to the plates, which has a maximum length of $2 m_{\mathrm{EM}}^{\prime} v$ for $\vartheta=0^{\circ}$ and $\vartheta=180^{\circ}$ and vanishes for $\vartheta=90^{\circ}$ and $\vartheta=270^{\circ}$. In the same first-order approximation, the spatial part of Eq. (85), the momentum associated with the stresses of the stabilizing mechanism, is

$$
\mathbf{P}_{\mathrm{stab}} \approx-m_{\mathrm{EM}}^{\prime} v(\cos 2 \vartheta, \sin 2 \vartheta, 0)
$$

This is a vector of constant length $m_{\mathrm{EM}}^{\prime} v$ in the direction $180^{\circ}+2 \vartheta$, as shown 
in Fig. 5 for $\vartheta=30^{\circ}$ and $\vartheta=60^{\circ} .{ }^{44}$
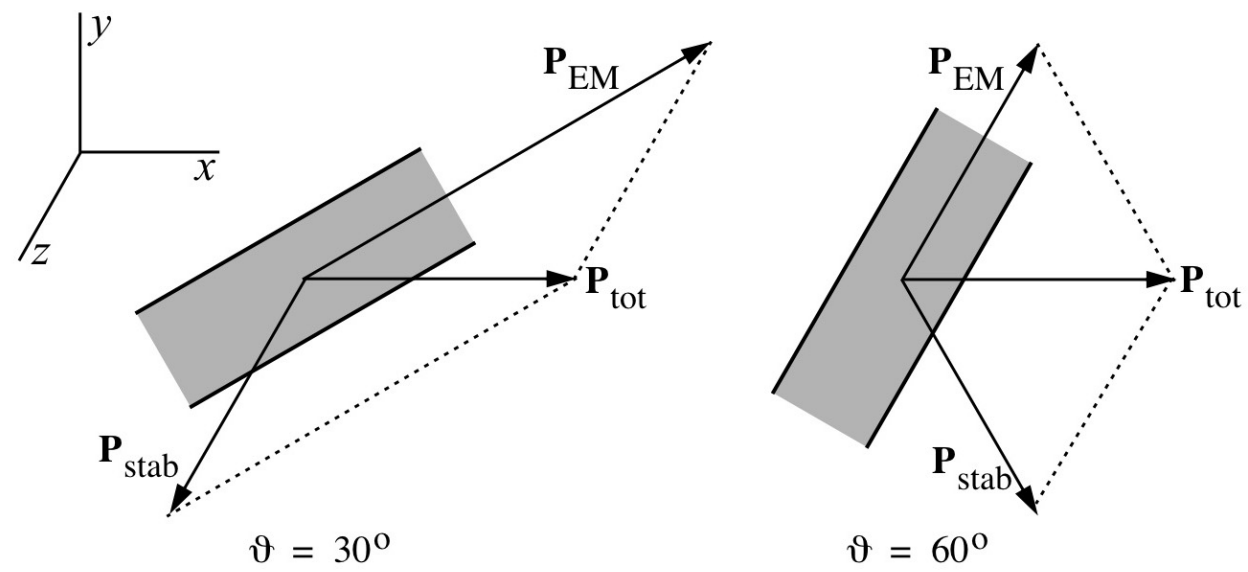

Fig. 5. Momentum of electromagnetic field and stabilizing mechanism in moving capacitor for two different values of $\vartheta$.

To bring out the peculiar features of these $\vartheta$-dependent expressions for energy and momentum more vividly, imagine that the moving capacitor is set rotating around the $z$-axis (i.e., the angle $\vartheta$ varies). Since there is no net torque, the capacitor will continue to rotate indefinitely without any further input of energy. An observer with respect to whom the capacitor is moving at velocity $v$ in the $x$-direction will conclude from Eqs. (84)-(85) and Eqs. (88)-(89) that, as the capacitor is adiabatically spinning around, energy and momentum flow back and forth between the electromagnetic field and the stabilizing mechanism. These exchanges of energy and momentum are puzzling until one realizes that, like the delicately balanced torques on the moving capacitor, they are simply artifacts of the convention for choosing spacelike hyperplanes that leads to the standard definition (41) of the four-momentum of spatially extended systems in Minkowski space-time. Unlike the flow of energy and momentum from the battery to the capacitor in the Trouton experiment, the flow of energy and momentum in this variation on the Trouton-Noble experiment can simply be defined out of existence.

\subsection{Drawing the line between kinematics and dynamics in accounting for the Trouton-Noble experiment}

The detailed account of what happens in the moving capacitor strongly depends on how one defines the four-momentum of spatially extended systems. On the standard definition (41), only the four-momentum of closed systems

\footnotetext{
${ }^{44}$ The diagrams for $\vartheta=120^{\circ}$ and $\vartheta=150^{\circ}$ are obtained by taking the mirror images in the $x z$-plane in Fig. 5 of the diagrams for $\vartheta=60^{\circ}$ and $\vartheta=30^{\circ}$, respectively.
} 
transforms as a four-vector under Lorentz transformations. On the alternative definition (42), championed by Rohrlich and others, this is true for all systems, closed or open. The difference between the two definitions lies in a convention for the selection of spacelike hyperplanes (cf. Fig. 2). There does not seem to be any compelling reason to prefer one convention over the other.

If one opts for the standard convention-picking hyperplanes of simultaneity in the rest frame of the observer - the account of what happens in the moving capacitor in the Trouton-Noble experiment involves a number of peculiar effects. The lack of covariance of the four-momentum of the open subsystems of the closed static system studied in the experiment manifests itself in various ways. The energy of the electromagnetic field and the energy of the stabilizing mechanism depend on the orientation of the capacitor with respect to its velocity but their sum remains constant. The equal and opposite stresses in the capacitor's rest frame result in equal and opposite momenta in a frame in which it is moving. These momenta are typically not in the direction of motion and give rise to two delicately balanced torques. None of these effects are specific to the Trouton-Noble experiment. They have the same kind of universality as length contraction, time dilation, and the increase of mass with velocity. One would find all three effects in any closed static system that can be divided in one way or another into static open subsystems. So the effects are kinematical in the broad sense. This is nicely illustrated by the observation, first made by Laue (1912a), that the capacitor in the Trouton-Noble experiment can be seen as a physical instantiation of a thought experiment of Lewis and Tolman (1909) involving a bent lever.

The three effects are also kinematical in the narrow sense of exemplifying the standard spatio-temporal behavior of special relativity. This follows from the observation that none of these effects occur if one opts for the alternative definition of four-momentum-picking hyperplanes of simultaneity in the rest frame of the capacitor. This suggests a more general sufficient condition for identifying relativistic effects as kinematical in the narrow sense: if an effect can be defined away by a mere change of convention about how to slice Minkowski space-time, then that effect is purely kinematical. The torques in the standard account of the Trouton-Noble experiment are kinematical by this criterion, as are length contraction and time dilation. In the case of length contraction, this can be seen directly in Fig. 2. Define the length of the arbitrary object shown in this figure as the length of some cross-section of its world sheet. The standard convention is to consider the intersection of the world sheet with hyperplanes of simultaneity in the observer's rest frame. In that case, the object moving at velocity $v$ is a factor $\gamma$ shorter in the direction of motion than the same object at rest. If, however, one adopts the alternative convention and considers the intersection of the world sheet with hyperplanes of simultaneity in the object's rest frame, there is no length contraction. This would seem to be a particularly compelling way to argue that length contrac- 
tion is a kinematical effect. If one wants to accord it the status of a dynamical effect, one has to privilege the standard convention for slicing world sheets. This is as true for the peculiar effects one encounters in the standard account of the Trouton-Noble experiment as it is for length contraction.

Ether theorists like Larmor and Lorentz, in effect, did privilege the standard convention. They adhered to Newtonian absolute simultaneity, which in relativistic terms amounts to privileging the hyperplanes of simultaneity of an observer at rest in their immobile universal ether. Faced with the contraction of the moving interferometer in the Michelson-Morley experiment or the delicately balanced torques on the moving capacitor in the Trouton-Noble experiment, they did not have the option of defining these effects out of existence by slicing space-time differently and understandably treated them as dynamical effects. Once the ether has been jettisoned along with Newtonian space and time, there no longer is a preferred convention. Applying the general criterion formulated above, one is then driven to the conclusion that the contractions, torques, and other contortions in the standard relativistic accounts of the Michelson-Morley experiment and the Trouton-Noble experiment are all purely kinematical effects.

\section{Kinematical explanations and Minkowski space-time}

The analyses in secs. 2-3 follow a simple pattern. Dynamical considerations specific to the physical system at hand are brought in to account for some feature(s) of the system at rest - be it with respect to the ether, as in Lorentz's theory, or with respect to some arbitrary inertial frame, as in Einstein's special theory of relativity. Once this task has been accomplished for the relevant feature(s) and to the desired level of specificity, an appeal to the Lorentz invariance of the laws and models used in these accounts suffices to account for any associated features of the same system in uniform motion. In Lorentz's theory, Lorentz invariance is a symmetry of all dynamical laws but not of the space-time structure posited by the theory. ${ }^{45}$ In special relativity it is both. Switching from an active to a passive reading of the Lorentz transformation for a moment, the relativist can thus look upon the boosted version of the situation at rest simply as a redescription of the same situation from the vantage point of an observer with respect to whom the system is moving. This observation makes it clear that the relevant features of the moving system do not call for a dynamical explanation in special relativity. They are purely kinematical in the sense that the moving system is just exhibiting default spatio-temporal behavior. In Lorentz's theory, they are kinematical only in

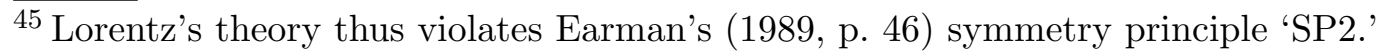


the broad sense of being independent of the details of the dynamics (even though Lorentz did not recognize this right away in all cases).

In sec. 2, the system at rest is some transparent medium and what calls for a dynamical account is the value of $n$, its refractive index. The Lorentz invariance of that account guarantees that, if the medium is moving with velocity $v$, light propagating through it in the direction of motion will have velocity $(c / n)+f v$, where $f \equiv 1-1 / n^{2}$ is the Fresnel drag coefficient. This Fresnel "drag" is thus a purely kinematical effect. The Lorentz-invariant classical account of refraction and dispersion in (Lorentz, 1892a) was eventually superseded by a quantum-mechanical account, but, since that account is Lorentz invariant too, it likewise yields the Fresnel drag coefficient. As Laue (1907) showed, the drag coefficient provides an example of how velocities add in special relativity. It tells us nothing about the way in which light propagates through transparent media. Physicists in the 1890s were therefore mistaken in thinking that it is further evidence for Lorentz's theory of refraction and dispersion that it yields the Fresnel drag coefficient.

In sec. 3, the 'system' at rest is the classical electron of Lorentz and Poincaré and the task is to account for its inertia (the scare quotes serve as a reminder that electrons cannot be modeled the way Lorentz and Poincaré envisioned). This task amounts to finding all contributions to its rest energy. In modern physics, one simply takes the rest mass $m^{\prime}$ of the electron as a primitive parameter. Either way, one arrives at the expression $P^{\prime \mu}=\left(m^{\prime} c, 0,0,0\right)$ for the four-momentum of the electron at rest. An electron moving with velocity $\mathbf{v}$ then has four-momentum $P^{\mu}=\left(\gamma m^{\prime} c, \gamma m^{\prime} \mathbf{v}\right)$, the Lorentz-boosted version of $P^{\prime \mu}$. If one defines mass as the ratio of momentum and velocity (as is customary nowadays), the mass of a moving electron is $\gamma \mathrm{m}^{\prime}$. If one defines it as the ratio of force and acceleration (as was customary around 1905), the electron has two different masses depending on whether the acceleration is in the direction of the velocity or perpendicular to it. These two masses are the longitudinal mass, $m_{/ /}=\gamma^{3} m^{\prime}$, and the transverse mass, $m_{\perp}=\gamma m^{\prime}$, respectively. Either way, the increase of mass with velocity given by these expressions is a purely kinematical effect. Contrary to what physicists thought around 1905, the velocity dependence of the electron's mass tells us nothing about the nature or structure of the electron, but only about the behavior of the four-momentum of a closed system - any closed system - under Lorentz transformations (secs. 3.4-3.5). In other words, it is no evidence for the Lorentz-Poincaré model of the electron that this model exhibits the generic velocity dependence of mass in special relativity.

In sec. 4, the system at rest is a charged capacitor and the task is to account for its stability. It would not be easy to produce a realistic quantum-mechanical model of the stabilizing mechanism. Fortunately, it suffices to derive the form of its energy-momentum tensor from the requirement that the capacitor and 
its electromagnetic field taken together form a closed static system. This gives a Lorentz-invariant model of a charged capacitor at rest, even though the model does not specify the details of the physics behind the stabilizing mechanism. Performing a Lorentz transformation, using the standard definition of the four-momentum of spatially extended systems, one finds that the delicate balance between electromagnetic field and stabilizing mechanism in the capacitor at rest translates into a number of peculiar effects in the capacitor in motion. The way in which the total energy, momentum, and angular momentum is divided between field and stabilizing mechanism in the moving capacitor depends on its orientation with respect to its velocity. This results in two equal and opposite torques acting on the system. It also results in a flow of energy, momentum, and angular momentum between the two components of the system every time the system's orientation is changed. One does not find any of these effects if one adopt a different convention for choosing space-like hyperplanes $\Sigma$ in the definition of the four-momentum $P_{\Sigma}^{\mu}$ of spatially extended systems (cf. Fig. 2). This is a particularly vivid illustration of the kinematical character of these effects. The kinematical character of length contraction and time dilation can be argued for in the exact same way (sec. 4.4).

Lorentz invariance manifests itself in a number of generic features of moving systems: length contraction, time dilation, a new addition rule for velocities, the velocity dependence of mass, and delicately balanced torques coming from open subsystems of closed systems. In special relativity - at least in the standard version - Lorentz invariance in turn is interpreted as reflecting a symmetry of Minkowski space-time, the space-time structure posited by the theory. In several places (Janssen, 1995, 2002a,b; Balashov and Janssen, 2003), I have presented what I have come to call a common origin inference (COI) from universal Lorentz invariance to Minkowski space-time. Both in Lorentz's theory and in Brown's alternative interpretation of special relativity (Norton, 2007), Lorentz invariance is posited separately for different dynamical laws. The systems studied in secs. 2-4 illustrate this point. They all involve the interaction between electric charges and electromagnetic fields on the one hand and matter keeping these charges in place on the other. In these three examples, the role of matter is played by the transparent medium, the Poincaré ether pressure, and the capacitor, respectively. Lorentz introduced the Lorentz invariance of the laws governing the electromagnetic fields and the electric charges through his theorem of corresponding states. In modern terms, he proved that Maxwell's equations, the known laws of electromagnetism, are Lorentz invariant. He then added what Brown and I call the generalized deformation hypothesis (sec. 3.1), which is the assumption that the laws governing everything else are Lorentz invariant as well. In Lorentz's theory and mutatis mutandis in Brown's proposal, it is simply a brute fact that these different laws are Lorentz invariant. In the orthodox version of special relativity this cosmic coincidence is traced to a common origin, Minkowski space-time. 
The account of the Michelson-Morley experiment in Lorentz's theory provides another illustration of how this COI works. It is a direct consequence of the Lorentz invariance of the source-free Maxwell equations that the Lorentzboosted version of any pattern of brightness and darkness is contracted by a factor $\gamma$ in the direction of motion compared to the original pattern at rest. For this contraction to be undetectable for a co-moving observer, it has to be the case that moving Michelson interferometers and other arrangements of optical components used to produce and observe patterns of brightness and darkness experience the same contraction (sec. 3.1). This will be the case if the laws governing these systems are Lorentz invariant. In the Michelson-Morley experiment of 1887, the optical components were all mounted on a slab of sandstone. To account for the negative result of the experiment it thus had to be assumed that sandstone moving through the ether is subject to the LorentzFitzGerald contraction. In Michelson's original experiment, the interferometer was made of brass, so it had to be assumed that brass experiences the same contraction as sandstone (Janssen, 2002b, p. 498).

Astonishingly, in the years 1900-1905, Morley and a new collaborator, Miller, performed a series of experiments to test this assumption for other materials such as pine wood and steel (Swenson, 1972, Ch. 7). These experiments provide a textbook example of the waste of time and resources that can occur if experimenters rely on faulty explanations to guide them in their work (sec. 1.1). Morley and Miller were badly out of touch with theoretical developments when they did these experiments. Even though nobody before Einstein and Minkowski appreciated that length contraction is kinematical in the narrow sense of being an example of default spatio-temporal behavior, any competent theorist in the late 1890s and early 1900s realized that length contraction is kinematical in the broad sense of being independent of the details of the dynamics. By my book, the Morley-Miller experiments are therefore almost as embarrassing as Miller's attempts in the early 1920s to repeat the MichelsonMorley experiment on Mount Wilson, on the argument that the rooms in which the earlier experiments had been done might have trapped the ether (Swenson, 1972, Ch. 10). Brown, however, evaluates these Morley-Miller experiments much more positively:

We are now so used to this miracle [that moving systems contract by a factor $\gamma$ in the direction of motion regardless of what they are made of] that it seems mundane, but it is worth recalling that in the early twentieth century the Michelson-Morley experiment was repeated on several occasions with different substances making up the rigid support of the interferometer mirrors precisely to test for the universality of the FitzGerald-Lorentz deformation effect (Brown, 2005, p. 30).

Special relativity traces the "miracle" that brass, stone, steel, wood, and electromagnetic field configurations all experience the same contraction to the 
structure of Minkowski space-time. As Dorling used to tell me, the universality of the Lorentz-FitzGerald contraction in special relativity is no more miraculous than that right-angled triangles cut out of flat sheets of paper, plastic, and card board all satisfy the Pythagorean theorem of Euclidean geometry. To avoid misunderstandings, let me emphasize that Brown is quite right in the sense that it is a highly non-trivial fact about nature that spacetime can be taken to be Minkowskian in a great many situations or, for that matter, that space can usually be taken to be Euclidean.

Brown and Pooley resist the COI from universal Lorentz invariance to Minkowski space-time. COI is a subspecies of IBE. So it is my view that Minkowski space-time explains Lorentz invariance. For Brown and Pooley, however, Minkowski space-time is a "glorious non-entity" that can do no explanatory work. I already disavowed the notion that Minkowski space-time be a substance with causal efficacy, so the sense in which Minkowski space-time explains Lorentz invariance is certainly not causal. I adopted the view, similar to Brown's, that Minkowski space-time encodes the default spatio-temporal behavior of all physical systems in a world in accordance with the laws of special relativity (sec. 1.2). The challenge of (Brown, 2005) and (Brown and Pooley, 2006) for my position then is twofold. First, how can Minkowski space-time do any explanatory work if it is not a substance? As Brown writes commenting on the statement of the COI to Minkowski space-time in (Balashov and Janssen, 2003): "Here we are at the heart of the matter. It is wholly unclear how this geometrical explanation is supposed to work" (Brown, 2005, p. 134). Elaborating on this complaint a little later in the book, he calls this issue the "mystery of mysteries" (ibid., p. 143). Second, on my own non-substantival conception of Minkowski space-time, does the statement that space-time is Minkowskian say anything over and above the statement that all laws are Lorentz invariant? The two questions can be rolled into one: where exactly does the explanatory gain come from that I claim special relativity in its orthodox form has both over Lorentz's theory and over Brown's etherless, preferred-frame-less version of same?

Let me first dispose of one possible answer to the question of explanatory gain. Special relativity certainly does not explain why space-time is Minkowskian. That is a brute fact in the theory. It follows that the orthodox relativist also does not have an answer to the question, which Brown (2005, e.g., p. 14-15, pp. 23-24, pp. 140-142) considers particularly pressing, of why free particles move on geodesics of Minkowski space-time other than to point out that it is part and parcel of the claim that space-time is Minkowskian that they do. As many have argued, Minkowski space-time is a very natural space-time structure, but such arguments invariably assume other brute facts that would seem to stand in just as much need of explanation. Dorling (1976), for instance, shows how one might have arrived at Minkowski's space-time geometry in antiquity through a simple modification of one axiom of Euclidean geometry that would 
have to be modified in "any geometry which was intended to embrace time as well as space" (quoted from the abstract of Dorling's still unpublished paper). Minkowski (1909, p. 79) famously remarked that a mathematician might have arrived at a geometry characterized by the Lorentz group well in advance of the developments in electrodynamics that historically led to the recognition of this group's importance by noting that classical mechanics is invariant under transformations both of the Euclidean and of the Galilean group.

So what is the explanatory advantage of special relativity over the alternatives of Lorentz and Brown and how does it come about? Theories like special relativity that are about the spatio-temporal behavior of physical systems assign all phenomena to one of two classes, the class of kinematical and the class of dynamical phenomena. The former contains all phenomena that are simply examples of the default or generic spatio-temporal behavior posited by the theory. In special relativity, the set of norms defining this default behavior is encoded in the geometry of Minkowski space-time. Phenomena assigned to this class do not call for any further explanation. The only phenomena calling for further explanation are those assigned to the class of dynamical phenomena. These phenomena involve systems exhibiting spatio-temporal behavior that is not generic, either because it is system-specific (e.g., why is the speed of light in this transparent medium $c / n$ ?) or because it deviates from the norm (e.g., why is the trajectory of this particle not a geodesic?). As the examples discussed in this paper illustrate, many phenomena that were classified as dynamical in Lorentz's theory and in Brown's proposal (the Fresnel drag coefficient in the Fizeau experiment, the velocity dependence of the electron mass in the experiments of Kaufmann and others, and the torques on the moving capacitor in the Trouton-Noble experiment) are reclassified as kinematical in special relativity.

Using this way of capturing the kinematics-dynamics distinction, I can now give an answer to the question how Minkowski space-time (understood as encoding the default spatio-temporal behavior of special relativity) explains such phenomena as length contraction and the velocity dependence of mass. It explains them by showing they need no explanation. Or, to put it less paradoxically, the statement that space-time is Minkowskian explains all of them in one fell swoop. This then is where that statement goes beyond the statement that all laws are Lorentz invariant. It commits one to assigning all manifestations of Lorentz invariance to the class of kinematical phenomena. Special relativity thus achieves its explanatory gain over Lorentz's theory by redrawing the line between kinematics and dynamics. Since explanation provides a guide to inference (sec. 1.1), this makes a difference in scientific practice. It yields a negative and a positive heuristic (sec. 3.5). It tells scientists that there is nothing more to be learned from the study of specific elements in the class of kinematical phenomena. The criteria defining the class of kinematical phenomena, however, provide useful constraints on further theorizing about 
elements in the class of dynamical phenomena.

It does not follow from any of this that it is fruitless to ask why space-time is Minkowskian and to search for a deeper explanation of Lorentz invariance and other features it encodes. The COI from universal Lorentz invariance to Minkowski space-time (again: understood as the geometrical encoding of a new kinematics) does tell us, however, that it would be an unprecedented reversal of fortunes in the history of science if the ultimate explanation of Lorentz invariance would be that all dynamical laws just happen to be Lorentz invariant. And this is the Lorentz 1899/1904 position that Brown suggests we return to (albeit sans ether or absolute simultaneity).

Proponents of the electromagnetic view of nature discussed in sec. 3 actually pursued a more respectable variant of this explanation (Janssen, 2002b, pp. 498-499). Minkowski space-time is not the only possible common origin for the Lorentz invariance of laws governing different interactions. Another option is that it is a property of some unified theory for these interactions. This is what proponents of the electromagnetic view of nature suggested. At first, the unifying theory was taken to be classical electrodynamics itself. In the 1910s, Mie replaced it by some non-linear generalization of Maxwell's theory. By 1920, if not earlier, it was clear that this electromagnetic program was not panning out. With the program's demise the alternative common origin for universal Lorentz invariance was eliminated.

In the two theories combined in today's standard model, the Weinberg-Salam theory and quantum chromodynamics (QCD), Lorentz invariance is assumed from the start as reflecting the common space-time background of these theories. This illustrates how special relativity imposes a kinematical constraint on dynamical theories, a meta-law in the language of (Lange, 2007). For a relationist, the laws of these two theories are the bearers of the property of Lorentz invariance, but this property has nothing to do with the specifics of the electroweak or the strong interaction. Explaining Lorentz invariance in terms of such specifics would thus be to misidentify its origin, potentially sending us off on a wild goose chase. General relativity tells us that Minkowski space-time, or rather its metric field, is a particular field configuration of the inertio-gravitational field. This is unlikely to be the final word on the matter. We can imagine that Minkowski space-time will emerge in the low-energy limit of some future theory of quantum gravity that does not include any spatiotemporal notions among its basic concepts. Such a theory would provide an answer to the question 'Why Minkowski space-time?'. There is no reason to think, however, that this deeper theory would require us to move any of the phenomena in which Lorentz invariance manifests itself from the column of kinematics to the column of dynamics as established by special relativity. To the extent that Brown is urging that we keep looking for a deeper explanation of Lorentz invariance than Minkowski space-time, I have no beef with him at 
all. I hope to have shown in this paper, however, that such a deeper explanation is not to be had by a return to the borders between kinematics and dynamics as they were before 1905 .

This illustrates a general feature of COIs. COIs are often to new taxonomies of what Laura Snyder has proposed to call "phenomena kinds" (in analogy with "natural kinds"). Taxonomies arrived at or backed up by a strong COI tend to be robust enough to survive radical theory change (Janssen 2002b, p. 492; Snyder, 2006, p. 184). The COI to Minkowski space-time is a case in point. The inference is not to some substance with causal efficacy (i.e., it is not a common cause inference) but to a new classification of phenomena as kinematical and dynamical. This new constraint on how nature can and cannot be "carved at its joints" is unlikely to be lifted by further theoretical developments.

To conclude this paper, I want to draw attention to another episode in which the kinematics-dynamics distinction (in the broad sense) played a crucial role. Prior to 1905, it had looked as if Maxwell's dynamical equations were incompatible with the relativity principle. Einstein (1905a) showed how a new space-time kinematics took care of that problem. At the time, it also looked as if Maxwell's equations were incompatible with Planck's black-body radiation law and the wave-particle duality suggested by its implications for energy and momentum fluctuations in black-body radiation. Jordan showed in 1925 how the new kinematics that Heisenberg (1925) introduced in his Umdeutung paper (kinematics in the sense of a new general framework for physics) made that problem go away (Duncan and Janssen, 2007b). As it says in the final section of the so-called Dreimännerarbeit, in which both terms of Einstein's 1909 fluctuation formula, the wave and the particle term, are derived for a simple model of a cavity filled with black-body radiation: "The basic difference between the theory proposed here and that used hitherto ... lies in the characteristic kinematics and not in a disparity of the mechanical laws" (Born, Heisenberg, and Jordan, 1926, p. 385). As Jordan reiterated triumphantly in a paper two years later: "We explicitly stuck to the wave theory of light and only changed the kinematics of cavity waves quantum-mechanically. From this, however, the characteristic light-quantum effects emerged automatically as a consequence" (Jordan, 1928, p. 195).

\section{Acknowledgments}

This paper grew out of a talk for the symposium 'Time and Relativity' devoted to issues raised by Harvey Brown's Physical Relativity and held at the Institute for Advanced Study (IAS) of the University of Minnesota, October 26-27, 2007. I want to thank Angie Hoffmann-Walter, Karen Kinoshita, Su- 
sannah Smith, and Ann Waltner of the IAS for organizing this symposium. Antigone Nounou and I put together the scientific program and led a reading group in preparation of the symposium. This paper owes much to her wise counsel. It also benefited greatly from helpful feedback from participants in both the reading group and the symposium as well as from the audience at a colloquium on the same material at the University of Maryland, November 5, 2007. My discussions with Harvey Brown and Oliver Pooley at the IAS symposium were particularly helpful. I am indebted to Yuri Balashov, Jeff Bub, Jed Buchwald, Dennis Dieks, Jon Dorling, Tony Duncan, Gordon Fleming, Gerd Graßhoff, Amit Hagar, Don Howard, Anne Kox, John Norton, Jürgen Renn, Serge Rudaz, Rob Rynasiewicz, Merrilee Salmon, John Stachel, and the late great Wes Salmon for useful advice over the years on various components of this paper. Dea mea invita opus conficere non poteram.

\section{References}

Abraham, M. (1902a). Dynamik des Elektrons. Königliche Gesellschaft der Wissenschaften zu Göttingen. Mathematisch-physikalische Klasse. Nachrichten: 20-41.

Abraham, M. (1902b). Dynamik des Elektrons. Königliche Gesellschaft der Wissenschaften zu Göttingen. Mathematisch-physikalische Klasse, Nachrichten, pp. 20-41.

Abraham, M. (1903). Prinzipien der Dynamik des Elektron. Annalen der Physik 10: 105-179.

Abraham, M. (1905). Theorie der Elektrizität. Vol. 2. Elektromagnetische Theorie der Strahlung, Teubner: Leipzig.

Balashov, Y. and Janssen, M. (2003). Presentism and relativity. British Journal for the Philosophy of Science 54: 327-346.

Bellon, R. D. (2006). Joseph Hooker takes a "fixed post": Transmutation and the "present unsatisfactory state of systematic botany", 1844-1860. Journal of the History of Biology 39: 1-39

Born, M., Heisenberg, W., and Jordan, P. (1926). Zur Quantenmechanik II. Zeitschrift für Physik 35: 557-615. Page references to English translation in (Van der Waerden, 1968, pp. 321-385).

Brown, H. R. (2005). Physical relativity. Space-time structure from a dynamical perspective, Oxford: Oxford University Press.

Brown, H. R. and Pooley, O. (2001). The origins of the spacetime metric: Bell's Lorentzian pedagogy and its significance in general relativity. Pp. 256-272 in: C. Callender and N. Huggett (eds.), Physics meets philosophy at the Planck scale. Cambridge: Cambridge University Press.

Brown, H. R. and Pooley, O. (2006). Minkowski space-time: A glorious nonentity. Pp. 67-92 in: D. Dieks (ed.), The ontology of spacetime. Amsterdam: Elsevier. 
Bub, J. and Pitowsky, I. (2007). Two dogmas about quantum mechanics. To appear in a volume based on the proceedings of the conference Everett@50 at Oxford University, July 19-21, 2007.

Buchwald, J. Z. (ed.) (1995). Scientific practice. Theories and stories of doing physics. Chicago: University of Chicago Press.

Butler, J. W. (1968). On the Trouton-Noble experiment. American Journal of Physics 36: 936-941.

Campos, I. and Jiménez, J. L. (1986). Comment on the $4 / 3$ problem in the electromagnetic mass and the Boyer-Rohrlich controversy. Physical Review D 33: 607-610.

Corry, L. (1997). Hermann Minkowski and the postulate of relativity. Archive for History of Exact Sciences 51: 273-314.

Cushing, J. T. (1981). Electromagnetic mass, relativity, and the Kaufmann experiments. American Journal of Physics 49: 1133-1149.

Dorling, J. (1976]. Special relativity out of Euclidean geometry. Unpublished manuscript.

Duncan, A. and Janssen, M. (2007a). On the verge of Umdeutung in Minnesota: Van Vleck and the correspondence principle. 2 Parts, Archive for History of Exact Sciences 61: 553-624, 625-671.

Duncan, A. and Janssen, M. (2007b). Pascual Jordan's resolution of the conundrum of the wave-particle duality of light. Submitted to Studies in History and Philosophy of Modern Physics.

Earman, J. (1989). World enough and space-time. Absolute versus relational theories of space and time, Cambridge, MA: MIT press.

Ehrenfest, P. (1907). Die Translation deformierbarer Elektronen und der Flächensatz. Annalen der Physik 23: 204-205.

Einstein, A. (1905a). Zur Elektrodynamik bewegter Körper. Annalen der Physik 17: 891-921. Reprinted as Doc. 23 in (Einstein, 1987-2006, Vol. 2). Page references to English translation in (Einstein et al., 1952, pp. 37-65).

Einstein, A. (1905b). Ist die Trägheit eines Körpers von seinem Energieinhalt abhänging? Annalen der Physik 18: 639-641. Reprinted as Doc. 24 in (Einstein, 1987-2006, Vol. 2). English translation in (Einstein et al., 1952, pp. 69-71).

Einstein, A. (1907a). Bemerkungen zu der Notiz von Hrn. Paul Ehrenfest: "Die Translation deformierbarer Elektronen und der Flächensatz." Annalen der Physik 23: 206-208. Reprinted as Doc. 44 in (Einstein, 1987-2006, Vol. 2).

Einstein, A. (1907b). Über die vom Relativitätsprinzip geforderte Trägheit der Energie. Annalen der Physik 23: 371-384. Reprinted as Doc. 45 in (Einstein, 1987-2006, Vol. 2).

Einstein, A. (1917). Über die spezielle und allgemeine Relativitätstheorie (Gemeinverständlich). Braunschweig: Vieweg. Reprinted as Doc. 42 in (Einstein, 1987-2006, Vol. 6). Page references to English translation: Relativity. New York: Crown Publishers, 1961.

Einstein, A. (1918). Nachtrag. [supplement to (Weyl, 1918).] Preußische 
Akademie der Wissenschaften (Berlin). Sitzungsberichte: 478. Reprinted as Doc. 8 in (Einstein, 1987-2006, Vol. 7).

Einstein, A. (1919). Time, space, and gravitation. The London Times, November 28, 1919. Reprinted as Doc. 26 in (Einstein, 1987-2006, Vol. 7). Page references to reprint (under the title: What is the theory of relativity?) in (Einstein, 1954, pp. 227-232)

Einstein, A. (1949). Autobiographical Notes. Pp. 1-95 in: P. A. Schilpp (ed.), Albert Einstein: Philosopher-scientist. Evanston, IL: Library of Living Philosophers.

Einstein, A. (1954). Ideas and opinions. New York: Crown Publishers.

Einstein, A. (1987-2006). The collected papers of Albert Einstein. 10 Vols. Edited by J. Stachel et al. Princeton: Princeton University Press.

Einstein, A. et al. (1952). The principle of relativity, New York: Dover.

Fermi, E. (1921). Sulla dinamica di un sistema rigido di cariche elettriche in moto translatorio. Nuovo Cimento 22: 199-207.

Fermi, E. (1922). Über einen Widerspruch zwischen der elektrodynamischen und der relativistischen Theorie der elektromagnetischen Masse. Physikalische Zeitschrift 23: 340-344.

FitzGerald, G. F. (1902). The scientific writings of the late George Francis FitzGerald. Edited by J. Larmor. Dublin: Hodges, Figgis \& Co; London: Longmans, Green \& Co.

Fleming, G. N. (2000). Reeh-Schlieder meets Newton-Wigner. Philosophy of Science 67 (Proceedings): S495-S515.

Goldberg, S. (1970). Abraham, Max. Pp. 23-25 in: C. C. Gillespie (ed.), Dictionary of scientific biography. Vol. I. New York: Charles Scribner's Sons.

Friedman, M. (1983). Foundations of space-time theories. Relativistic physics and philosophy of science, Princeton: Princeton University Press.

Heisenberg, W. (1925). Über die quantentheoretische Umdeutung kinematischer und mechanischer Beziehungen. Zeitschrift für Physik 33: 879-893. English translation in (Van der Waerden, 1968, pp. 261-276).

Hon, G. (1995). Is the identification of experimental error contextually dependent? The case of Kaufmann's experiment and its varied reception. in (Buchwald, 1995, pp. 170-223).

Janssen, M. (1995). A comparison between Lorentz's ether theory and Einstein's special theory of relativity in the light of the experiments of Trouton and Noble. PhD thesis. University of Pittsburgh. Available on-line at www.mpiwg-berlin.mpg.de/en/sources/index.html.

Janssen, M. (2002a). Reconsidering a scientific revolution: the case of Lorentz versus Einstein. Physics in Perspective 4: 421-446.

Janssen, M. (2002b). COI stories: Explanation and evidence in the history of science. Perspectives on Science 10: 457-522.

Janssen, M. (2003). The Trouton experiment, $E=m c^{2}$, and a slice of Minkowski space-time. Pp. 27-54 in: J. Renn et al. (eds.), Revisiting the foundations of relativistic physics: Festschrift in honor of John Stachel. Dordrecht: Kluwer. 
Janssen, M. and Mecklenburg, M. (2007). From Classical to Relativistic Mechanics: Electromagnetic Models of the Electron. Pp. 65-134 in V. F. Hendricks et al. (eds.), Interactions: mathematics, physics and philosophy, 18601930. Berlin: Springer.

Janssen, M. and Stachel, J. (2004). L'Ottica e l'elettrodinamica dei corpi in movimento. Pp. 363-379 in: Sandro Petruccioli et al. (eds.), Storia della scienza. Vol. 8. Rome: Istituto della Enciclopedia Italiana. English original, Optics and electrodynamics in moving bodies, to appear in: J. Stachel, Going critical, Dordrecht: Springer.

Jordan, P. (1928). Die Lichtquantenhypothese. Entwicklung und gegenwärtiger Stand. Ergebnisse der exakten Naturwissenschaften 7: $158-208$.

Lange, M. (2007). Laws and meta-laws of nature: Conservation laws and symmetries. Studies in History and Philosophy of Modern Physics 38: 457-481.

Larmor, J. (1902). Can convection through the aether be detected electrically? In (FitzGerald, 1902, pp. 566-569)

Laue, M. (1907). Die Mitführung des Lichtes durch bewegte Körper nach dem Relativitätsprinzip. Annalen der Physik 23: 989-990. Reprinted in (Laue, 1961, Vol. 1, pp. 113-114).

Laue, M. (1911a). Zur Dynamik der Relativitätstheorie. Annalen der Physik 35: 524-542. Page references to reprint in (Laue, 1961, Vol. 1, pp. 135-153).

Laue, M. (1911b). Das Relativitätsprinzip. Braunschweig: Vieweg.

Laue, M. (1912a). Bemerkungen zum Hebelgesetz in der Relativitätstheorie. Physikalische Zeitschrift 12: 1008-1010. Reprinted in (Laue, 1961, Vol. 1, pp. 162-164).

Laue, M. (1912b). Zur Theorie des Versuches von Trouton und Noble. Annalen der Physik 38: 370-384. Page references to reprint in (Laue, 1961, Vol. 1, pp. 168-182). .

Laue, M. von (1961). Gesammelte Schriften und Vorträge. 3 Vols. Braunschweig: Vieweg.

Lewis, G. N. and Tolman, R. C. (1909). The principle of relativity, and nonNewtonian mechanics. Philosophical Magazine 18: 510-523.

Lipton, P. (2004). Inference to the best explanation. 2nd ed. London and New York: Routledge.

Lorentz, H. A. (1892a). La théorie électromagnétique de Maxwell et son application aux corps mouvants. Archives Néerlandaises des Sciences Exactes et Naturelles 25: 363-552. Reprinted in (Lorentz, 1934-39, Vol. 2, pp. 164$343)$.

Lorentz, H. A. (1892b). De relatieve beweging van de aarde en den aether. Koninklijke Akademie van Wetenschappen te Amsterdam. Verslagen van de gewone vergaderingen der wis en natuurkundige afdeeling 1 (1892-1893): 74-79. English translation in (Lorentz, 1934-39, Vol. 4, pp. 219-223).

Lorentz, H. A. (1895). Versuch einer Theorie der electrischen und optischen Erscheinungen in bewegten Körpern, Leiden: Brill. Reprinted in (Lorentz, 1934-39, Vol. 5, pp. 1-138). 
Lorentz, H. A. (1899). Simplified theory of electrical and optical phenomena in moving bodies. Koninklijke Akademie van Wetenschappen te Amsterdam, Section of Sciences, Proceedings 1 (1898-99): 427-442. Reprinted, in a slightly revised French translation, in (Lorentz, 1934-39, Vol. 5, pp. 139$155)$.

Lorentz, H. A. (1904a). Weiterbildung der Maxwellschen Theorie. Elektronentheorie. In (Sommerfeld, 1904-1922, pp. 145-288).

Lorentz, H. A. (1904b). Electromagnetic phenomena in a system moving with any velocity smaller than that of light. Koninklijke Akademie van Wetenschappen te Amsterdam, Section of Sciences, Proceedings 6 (1903-04): 809831. Page references to reprint in (Einstein et al., 1952, pp. 11-34).

Lorentz, H. A. (1915). The theory of electrons and its applications to the phenomena of light and radiant heat. $2 \mathrm{~d}$ ed. Leipzig: Teubner.

Lorentz, H. A. (1927). Problems of modern physics. A course of lectures delivered in the California Institute of Technology. Boston: Ginn.

Lorentz, H. A. (1934-39). Collected papers. 9 Vols. Edited by P. Zeeman and A. D. Fokker. The Hague: Nijhoff.

Martínez, A. A. (2007). There's no pain in the Fitzgerald contraction is there? Studies in History and Philosophy of Modern Physics 38: 209-215.

McCormmach, R. (1970). H. A. Lorentz and the Electromagnetic View of Nature. Isis 61: 459-497.

Miller, A. I. (1981). Albert Einstein's special theory of relativity. Emergence (1905) and early interpretation (1905-1911). Reading, MA: Addison-Wesley. Page references to reprint: New York: Springer, 1998.

Minkowski, H. (1908). Die Grundgleichungen für die elektromagnetischen Vorgänge in bewegten Körpern. Königliche Gesellschaft der Wissenschaften zu Göttingen, Mathematisch-physikalische Klasse. Nachrichten pp. 53-111. Reprinted in (Minkowski, 1967, Vol. 2, 352-404).

Minkowski, H. (1909). Raum und Zeit. Physikalische Zeitschrift 20: 104-111. Page references to English translation in (Einstein et al., 1952, pp. 75-91).

Minkowski, H. (1967). Gesammelte Abhandlungen. 2 Vols. in one. Edited by D. Hilbert. New York: Chelsea.

Norton, J. D. (1992). Einstein, Nordström and the early demise of scalar, Lorentz-Covariant theories of gravitation. Archive for the History of Exact Sciences 45: 17-94.

Norton, J. D. (1999). Geometries in collision: Einstein, Klein, and Riemann. Pp. 128-144 in: J. J. Gray (ed.), The symbolic universe. Geometry and physics 1890-1930. Oxford: Oxford University Press.

Norton, J. D. (2004). Einstein's investigations of Galilean covariant electrodynamics prior to 1905. Archive for History of Exact Sciences 59: 45-105

Norton, J. D. (2007). Why constructive relativity fails. Preprint available electronically at $\langle$ philsci-archive.pitt.edu/archive/00003655〉.

Pais, A. (1982). 'Subtle is the Lord ...' The science and the life of Albert Einstein. Oxford: Oxford University Press.

Pauli, W. (1921). Relativitätstheorie. In (Sommerfeld, 1904-1922, pp. 539- 
775). Reprinted in facsimile, edited by D. Giulini: Berlin: Springer, 2000. Page references to English translation: Theory of relativity. London: Pergamon, 1958. Reprinted: New York: Dover, 1981.

Planck, M. (1906a). Das Prinzip der Relativität und die Grundgleichungen der Mechanik. Deutsche Physikalische Gesellschaft. Verhandlungen 8: 136-141. Reprinted in (Planck, 1958, Vol. 2, pp. 115-120).

Planck, M. (1906b). Die Kaufmannschen Messungen der Ablenkbarkeit der $\beta$-Strahlen in ihrer Bedeutung für die Dynamik der Elektronen. Deutsche Physikalische Gesellschaft. Verhandlungen 8: 418-432. Page references to reprint in Physikalische Zeitschrift 7 (1906): 753-759 (760-761: discussion). Also reprinted in (Planck, 1958, Vol. 2, pp. 121-135).

Planck, M. (1908). Bemerkungen zum Prinzip der Aktion und Reaktion in der allgemeinen Dynamik. Deutsche Physikalische Gesellschaft. Verhandlungen 6: 728-732. Reprinted in (Planck, 1958, Vol. 2, pp. 215-219).

Planck, M. (1958). Physikalische Abhandlungen und Vorträge. 3 Vols. Braunschweig: Vieweg.

Poincaré, H. (1906). Sur la dynamique de l'électron. Rendiconti del Circolo Matematico di Palermo 21: 129-175.

Rynasiewicz, R., and Renn, J. (2007). The turning point for Einstein's annus mirabilis. Studies in History and Philosophy of Modern Physics 37: 5-35.

Rohrlich, F. (1960). Self-energy and the stability of the classical electron. American Journal of Physics 28: 639-643.

Rohrlich, F. (1965). Classical charged particles: Foundations of their theory, Reading, MA: Addison-Wesley.

Singal, A. K. (1993). On the "explanation" of the null results of the TroutonNoble experiments. American Journal of Physics 61: 428-433.

Smith, G. (2001). J. J. Thomson and the electron, 1897-1899. Pp. 21-76 in: J. Z. Buchwald and A. Warwick (eds.), Histories of the electron. The birth of microphysics. Cambridge, MA: The MIT Press.

Snyder, L. J. (2006). Reforming philosophy. A Victorian debate on science and society. Chicago: University of Chicago Press.

Sommerfeld, A. (ed.) (1904-1922). Encyklopädie der mathematischen Wissenschaften, mit Einschluß ihrer Anwendungen. Vol. 5, Physik, part 2. Leipzig: Teubner, 1904-1922.

Sommerfeld, A. (1910a). Zur Relativitätstheorie I. Vierdimensionale Vektoralgebra. Annalen der Physik 32: 749-776. Reprinted in (Sommerfeld, 1968, Vol. 2, pp. 189-216).

Sommerfeld, A. (1910b). Zur Relativitätstheorie II. Vierdimensionale Vektoranalyse. Annalen der Physik 33: 649-689. Reprinted in (Sommerfeld, 1968, Vol. 2, pp. 217-257).

Sommerfeld, A. (1968). Gesammelte Schriften, 4 Vols. Edited by F. Sauter. Braunschweig: Vieweg.

Stachel, J. (1994). Changes in the concepts of space and time brought about by relativity. Pp. 141-162 in: C. C. Gould and R. S. Cohen (eds.), Artifacts, representation and social practice. Dordrecht: Kluwer, . 
Stachel J. (2005). Fresnel's (dragging) coefficient as a challenge to 19th century optics of moving bodies. Pp. 1-13 in: J. Eisenstaedt and A. J. Kox (eds.), The universe of general relativity. Boston: Birkhäuser.

Swenson, L. S., Jr. (1972). The ethereal aether. A history of the MichelsonMorley-Miller aether-drift experiments, 1880-1930. Austin: University of Texas Press.

Teukolsky, S. A. (1996). The explanation of the Trouton-Noble experiment revisited. American Journal of Physics 64: 1104-1106.

Trouton, F. T. (1902). The results of an electrical experiment, involving the relative motion of the earth and ether, suggested by the late professor FitzGerald. Transactions of the Royal Dublin Society 7: 379-384. Reprinted in (FitzGerald, 1902, pp. 557-565).

Trouton, F. T. and Noble, H. R. (1903). The mechanical forces acting on a charged electric condenser moving through space. Philosophical Transactions of the Royal Society 202: 165-181.

Van der Waerden, B. L., ed. (1968). Sources of quantum mechanics. New York: Dover.

Warwick, A. (1995). The sturdy protestants of science: Larmor, Trouton, and the earth's motion through the ether. In (Buchwald, 1995, pp. 300-343).

Weyl, H. (1918). Gravitation und Elektrizität. Preußische Akademie der Wissenschaften (Berlin). Sitzungsberichte: 465-478. English translation in (Einstein et al., 1952, pp. 201-216)

Wien, W. (1900). Über die Möglichkeit einer elektromagnetischen Begründung der Mechanik. Archives Néerlandais des Sciences Exactes et Naturelles 2: 96-107. Reprinted in Annalen der Physik 5 (1901): 501-513.

Zahar, E. (1989). Einstein's revolution: A study in heuristic. La Salle, IL: Open Court.

Zeeman, P. (1927). Expériences sur la propagation de la lumière dans des milieux liquides ou solides en mouvement. Archives Néerlandais des Sciences Exactes et Naturelles (IIIA) 10: 131-220. 